\title{
SEARCH FOR THE HIGGS BOSON PRODUCED IN ASSOCIATION WITH A $W$ BOSON AT CDF RUN II
}

\section{DISSERTATION}

\author{
Presented in Partial Fulfillment of the Requirements for \\ the Degree Doctor of Philosophy in the \\ Graduate School of The Ohio State University \\ By \\ Jason Michael Slaunwhite, B.S., M.S.$$
* * * * *
$$

The Ohio State University

2009

Dissertation Committee:

Approved by

Prof. Richard E. Hughes, Adviser

Prof. Brian L. Winer

Prof. Junko Shigemitsu

Prof. Ezekiel Johnston-Halperin

Adviser

Graduate Program in

Physics 


\begin{abstract}
We present a search for standard model Higgs boson production in association with a $W$ boson in proton-antiproton collisions $\left(p \bar{p} \rightarrow W^{ \pm} H \rightarrow \ell \nu b \bar{b}\right)$ at a center of mass energy of $1.96 \mathrm{TeV}$. $W H$ candidate events have a signature of a single lepton $\left(e^{ \pm} / \mu^{ \pm}\right)$, missing transverse energy, and two jets. The search looks for candidate events in approximately $2.7 \mathrm{fb}^{-1}$ of data recorded with the CDF II detector. The high- $p_{T}$ lepton $(\mathrm{e}, \mu)$ in the events provides a distinct signature for triggering and most of the events in the dataset come from high- $p_{T}$ lepton triggers. Our analysis improves on prior searches by including events recorded on the $\mathbb{E}_{T}+2$ Jets trigger with a lepton reconstructed as an isolated high- $p_{T}$ charged particle. We increase the sample purity by identifying ("tagging") long-lived b-hadrons in jets. A neural network combines distinguishing kinematic information into a function optimized for $W H$ sensitivity. The neural network output distributions are consistent with the standard model background expectations and we set limits upper limits on the rate of Higgs production. We set $95 \%$ confidence level upper limits on the $W H$ production cross section times branching ratio for Higgs masses from 100 to 150 $\mathrm{GeV} / \mathrm{c}^{2}$ and express our results as a ratio of the experimental limit to the theoretical Standard Model production rate. Our limits range from 3.6 (4.3 expected) to 61.1 (43.2 expected) for Higgs masses from 100 to $150 \mathrm{GeV} / \mathrm{c}^{2}$, respectively.
\end{abstract}




\section{ACKNOWLEDGMENTS}

Thanks to everyone who helped and supported me, especially: Mom and Dad, Chris Slaunwhite, Richard Hughes, Brian Winer, Keivn Lannon, Ben Kilminster, Wei-ming Yao, Jonathan Efron, Brandon Parks, Fred Kuehn, Patrick Smith, Aaron Sander, Mark Murphey, Jon Hendrickson, and Tony Link. 


\section{VITA}

25 April $1982 \ldots \ldots \ldots \ldots \ldots \ldots \ldots \ldots$. . . . Born - Binghamton, NY

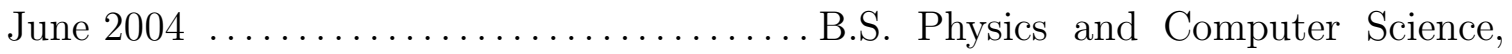
Union College, Schenectady, NY

September 2007 ....................... in Physics The Ohio State University, Columbus, $\mathrm{OH}$

\section{PUBLICATIONS}

"Search for Standard Model Higgs Boson Production in Association with a W Boson at CDF" T. Aaltonen et al. [CDF Collaboration] Phys. Rev. D 78, 032008 (2008) [arXiv:0803.3493 [hep-ex]]

"Search for Standard Model Higgs Bosons Produced in Association with W Bosons" T. Aaltonen et al. [CDF Collaboration] Phys. Rev. Lett. 100, 041801 (2008) [arXiv:0710.4363 [hep-ex]]

"Search for resonant $t \bar{t}$ production in $p \bar{p}$ collisions at $\sqrt{s}=1.96-\mathrm{TeV}$ " T. Aaltonen et al. [CDF Collaboration] Phys. Rev. Lett. 100, 231801 (2008) [arXiv:0709.0705 [hep-ex]]

"The CDF II level 1 track trigger upgrade" A. Abulencia et al. [CDF RUN II Collaboration] IEEE Trans. Nucl. Sci. 55, 126 (2008)

"The CDF II extremely fast tracker upgrade" A. Abulencia et al. [CDF Collaboration] Nucl. Instrum. Meth. A 572, 358 (2007)

"Search for Heavy, Long-Lived Neutralinos that Decay to Photons at CDF II Using Photon Timing" T. Aaltonen et al. [CDF Collaboration] Phys. Rev. D 78, 032015 (2008) [arXiv:0804.1043 [hep-ex]] 
"Search for the Higgs boson in events with missing transverse energy and $\mathrm{b}$ quark jets produced in proton-antiproton collisions at $\mathrm{s}^{* *}(1 / 2)=1.96$ TeV" T. Aaltonen et al. [CDF Collaboration] Phys. Rev. Lett. 100, 211801 (2008) [arXiv:0802.0432 [hep-ex]]

"Search for Pair Production of Scalar Top Quarks Decaying to a tau Lepton and a b Quark in ppbar Collisions at sqrts $=1.96 \mathrm{TeV}$ " T. Aaltonen et al. [CDF Collaboration] Phys. Rev. Lett. 101, 071802 (2008) [arXiv:0802.3887 [hep-ex]]

"Search for New Heavy Particles Decaying to $Z^{0} Z^{0} \rightarrow$ eeee in p-pbar Collisions at sqrt(s) $=1.96 \mathrm{TeV}$ " T. Aaltonen et al. [CDF Collaboration] Phys. Rev. D 78, 012008 (2008) [arXiv:0801.1129 [hep-ex]]

"Search for Heavy Top-like Quarks t' $\rightarrow$ Wq Using Lepton Plus Jets Events in $1.96 \mathrm{TeV}$ Proton-Antiproton Collisions" T. Aaltonen et al. [CDF Collaboration] Phys. Rev. Lett. 100, 161803 (2008) [arXiv:0801.3877 [hep-ex]]

"Measurement of Ratios of Fragmentation Fractions for Bottom Hadrons in p-pbar Collisions at sqrts $=1.96 \mathrm{TeV} " \mathrm{~T}$. Aaltonen et al. [CDF Collaboration] Phys. Rev. D 77, 072003 (2008) [arXiv:0801.4375 [hep-ex]]

"First Measurement of ZZ Production in ppbar Collisions at sqrt(s)=1.96 TeV" T. Aaltonen et al. [CDF Collaboration] Phys. Rev. Lett. 100, 201801 (2008) [arXiv:0801.4806 [hep-ex]]

"Observation of Exclusive Dijet Production at the Fermilab Tevatron ppbar Collider" T. Aaltonen et al. [CDF Run II Collaboration] Phys. Rev. D 77, 052004 (2008) [arXiv:0712.0604 [hep-ex]]

"Observation of the Decay $B_{c}^{ \pm} \rightarrow J / \psi \pi^{ \pm}$and Measurement of the $B_{c}^{ \pm}$ Mass" T. Aaltonen et al. [CDF Collaboration] Phys. Rev. Lett. 100, 182002 (2008) [arXiv:0712.1506 [hep-ex]]

"Search for $B_{s} \rightarrow \mu^{+} \mu^{-}$and $B_{d} \rightarrow \mu^{+} \mu^{-}$Decays with $2 \mathrm{fb}^{-1}$ of ppbar Collisions" T. Aaltonen et al. [CDF Collaboration] Phys. Rev. Lett. 100, 101802 (2008) [arXiv:0712.1708 [hep-ex]]

"Evidence for $D^{0}-\bar{D}^{0}$ bar mixing using the CDF II Detector" T. Aaltonen et al. [CDF Collaboration] Phys. Rev. Lett. 100, 121802 (2008) [arXiv:0712.1567 [hep-ex]] 
"Measurement of Lifetime and Decay-Width Difference in $B_{s}^{0} \rightarrow J / \Psi \phi$ Decays" T. Aaltonen et al. [CDF collaboration] Phys. Rev. Lett. 100, 121803 (2008) [arXiv:0712.2348 [hep-ex]]

"First Flavor-Tagged Determination of Bounds on Mixing-Induced CP Violation in $B_{s} \rightarrow J / \psi \phi$ Decays" T. Aaltonen et al. [CDF Collaboration] Phys. Rev. Lett. 100, 161802 (2008) [arXiv:0712.2397 [hep-ex]]

"Measurement of correlated b-bbar production in p-pbar collisions at $\mathrm{s}^{* *}(1 / 2)=1960 \mathrm{GeV}^{\prime}$ T. Aaltonen et al. [CDF Run II Collaboration] Phys. Rev. D 77, 072004 (2008) [arXiv:0710.1895 [hep-ex]]

"First Measurement of the Production of a W Boson in Association with a Single Charm Quark in Proton Anti-proton Collisions at $\operatorname{sqrt}(\mathrm{s})=\mathbf{1 . 9 6}$ TeV" T. Aaltonen et al. [CDF Collaboration] Phys. Rev. Lett. 100, 091803 (2008) [arXiv:0711.2901 [hep-ex]]

"Search for chargino-neutralino production in p-pbar collisions at 1.96 TeV with high pT leptons" T. Aaltonen et al. [CDF Collaboration] Phys. Rev. D 77, 052002 (2008) [arXiv:0711.3161 [hep-ex]]

"Measurement of Inclusive Jet Cross Sections in $Z / \gamma^{*}(\rightarrow e e)+$ jets Production in $p \bar{p}$ Collisions at $\sqrt{s}=1.96 \mathbf{T e V}^{\prime}$ T. Aaltonen et al. [CDF - Run II Collaboration] Phys. Rev. Lett. 100, 102001 (2008) [arXiv:0711.3717 [hep-ex]]

"Measurement of the cross section for W-boson production in association with jets in ppbar collisions at $\sqrt{s}=1.96 \mathrm{TeV}^{\prime}$ T. Aaltonen et al. [CDF Collaboration] Phys. Rev. D 77, 011108 (2008) [arXiv:0711.4044 [hep-ex]]

"First observation of the decay $B_{s}^{0} \rightarrow D_{s}^{-} D_{s}^{+}$and measurement of its branching ratio" T. Aaltonen et al. [CDF Run II Collaboration] Phys. Rev. Lett. 100, 021803 (2008)

"Cross Section Constrained Top Quark Mass Measurement from Dilepton Events at the Tevatron" T. Aaltonen et al. [CDF Collaboration] Phys. Rev. Lett. 100, 062005 (2008) [arXiv:0710.4037 [hep-ex]]

"A direct Measurement of the W Boson Width in ppbar Collisions at $\operatorname{sqrt}(\mathrm{s})=1.96 \mathrm{TeV} "$ T. Aaltonen et al. [CDF Collaboration] Phys. Rev. Lett. 100, 071801 (2008) [arXiv:0710.4112 [hep-ex]] 
"Observation of Orbitally Excited $B_{s}$ Mesons" T. Aaltonen et al. [CDF Collaboration] Phys. Rev. Lett. 100, 082001 (2008) [arXiv:0710.4199 [hep-ex]]

"Limits on the Production of Narrow t-tbar Resonances in p-pbar Collisions at $\operatorname{sqrt}(\mathrm{s})=\mathbf{1 . 9 6} \mathrm{TeV}^{\prime}$ T. Aaltonen et al. [CDF Collaboration] Phys. Rev. D 77, 051102 (2008) [arXiv:0710.5335 [hep-ex]]

"First Run II Measurement of the $W$ Boson Mass" T. Aaltonen et al. [CDF Collaboration] Phys. Rev. D 77, 112001 (2008) [arXiv:0708.3642 [hep-ex]]

"First Measurement of the W Boson Mass in Run II of the Tevatron" T. Aaltonen et al. [CDF Collaboration] Phys. Rev. Lett. 99, 151801 (2007) [arXiv:0707.0085 [hep-ex]]

"Observation and mass measurement of the baryon $\Xi_{b}^{-}$" T. Aaltonen et al. [CDF Collaboration] Phys. Rev. Lett. 99, 052002 (2007) [arXiv:0707.0589 [hep-ex]]

"Search for a high-mass diphoton state and limits on Randall-Sundrum gravitons at CDF" T. Aaltonen et al. [CDF Collaboration] Phys. Rev. Lett. 99, 171801 (2007) [arXiv:0707.2294 [hep-ex]]

"Search for chargino-neutralino production in $p \bar{p}$ collisions at $\sqrt{s}=1.96$ TeV" T. Aaltonen et al. [CDF Collaboration] Phys. Rev. Lett. 99, 191806 (2007) [arXiv:0707.2362 [hep-ex]]

"Search for exclusive $\gamma \gamma$ production in hadron-hadron collisions" T. Aaltonen et al. [CDF Collaboration] Phys. Rev. Lett. 99, 242002 (2007) [arXiv:0707.2374 [hep-ex]]

"Search for new physics in high mass electron-positron events in $p \bar{p}$ collisions at $\sqrt{s}=1.96-\mathrm{TeV}^{\prime}$ T. Aaltonen et al. [CDF Collaboration] Phys. Rev. Lett. 99, 171802 (2007) [arXiv:0707.2524 [hep-ex]]

"Search for Direct Pair Production of Supersymmetric Top and Supersymmetric Bottom Quarks in $p \bar{p}$ Collisions at $\sqrt{s}=1.96-\mathrm{TeV}^{\prime}$ T. Aaltonen et al. [CDF Collaboration] Phys. Rev. D 76, 072010 (2007) [arXiv:0707.2567 [hep-ex]]

"Search for New Particles Leading to $Z+$ jets Final States in $p \bar{p}$ Collisions at $\sqrt{s}=1.96-T^{\prime}{ }^{\prime}$ T. Aaltonen et al. [CDF Collaboration] Phys. Rev. D 76, 072006 (2007) [arXiv:0706.3264 [hep-ex]] 
"Measurement of the $p \bar{p} \rightarrow t \bar{t}$ production cross- section and the top quark mass at $\sqrt{s}=1.96-\mathrm{TeV}$ in the all-hadronic decay mode" T. Aaltonen et al. [CDF Collaboration] Phys. Rev. D 76, 072009 (2007) [arXiv:0706.3790 [hep-ex]]

"First observation of heavy baryons $\Sigma_{b}$ and $\Sigma_{b}^{*}$ " T. Aaltonen et al. [CDF Collaboration] Phys. Rev. Lett. 99, 202001 (2007) [arXiv:0706.3868 [hep-ex]]

"Measurement of the top-quark mass using missing $E_{T}+$ jets events with secondary vertex $b$-tagging at CDF II" T. Aaltonen et al. [CDF Collaboration] Phys. Rev. D 75, 111103 (2007) [arXiv:0705.1594 [hep-ex]]

"Limits on Anomalous Triple Gauge Couplings in $p \bar{p}$ Collisions at $\sqrt{s}=$ 1.96-TeV" T. Aaltonen et al. [CDF Collaboration] Phys. Rev. D 76, 111103 (2007) [arXiv:0705.2247 [hep-ex]]

"Polarization of $J / \psi$ and $\psi_{2 S}$ mesons produced in $p \bar{p}$ collisions at $\sqrt{s}=$ 1.96-TeV" A. Abulencia et al. [CDF Collaboration] Phys. Rev. Lett. 99, 132001 (2007) [arXiv:0704.0638 [hep-ex]]

"Search for heavy, long-lived particles that decay to photons at CDF II" A. Abulencia et al. [CDF Collaboration] Phys. Rev. Lett. 99, 121801 (2007) [arXiv:0704.0760 [hep-ex]]

"Measurement of $\sigma_{\chi_{c 2}} \mathcal{B}\left(\chi_{c 2} \rightarrow J / \psi \gamma\right) / \sigma_{\chi_{c 1}} \mathcal{B}\left(\chi_{c 1} \rightarrow J / \psi \gamma\right)$ in $p \bar{p}$ collisions at $\sqrt{s}$ = 1.96-TeV" A. Abulencia et al. [CDF Run II Collaboration] Phys. Rev. Lett. 98, 232001 (2007) [arXiv:hep-ex/0703028]

"Precise measurement of the top quark mass in the lepton+jets topology at CDF II" A. Abulencia et al. [CDF Collaboration] Phys. Rev. Lett. 99, 182002 (2007) [arXiv:hep-ex/0703045]

"Observation of $W Z$ Production" A. Abulencia et al. [CDF Collaboration] Phys. Rev. Lett. 98, 161801 (2007) [arXiv:hep-ex/0702027]

"Search for new physics in lepton + photon $+X$ events with $929 \mathrm{pb}^{(-1)}$ of $p \bar{p}$ collisions at $\sqrt{s}=1.96-\mathrm{TeV}^{\prime}$ A. Abulencia et al. [CDF Run II Collaboration] Phys. Rev. D 75, 112001 (2007) [arXiv:hep-ex/0702029] 
"First Measurement of the Ratio of Central-Electron to Forward-Electron W Partial Cross Sections in p-pbar Collisions at sqrts $=1.96$ TeV" A. Abulencia et al. [CDF Collaboration] Phys. Rev. Lett. 98, 251801 (2007) [arXiv:hepex/0702037]

"Measurement of $\sigma(p \bar{p} \rightarrow \mathrm{Z}) \cdot \mathrm{Br}(\mathrm{Z} \rightarrow 2$ tau $)$ in $\mathrm{p}$ anti-p collisions at $\mathrm{s}^{* *}(1 / 2)=1.96 \mathrm{TeV} "$ A. Abulencia et al. [CDF Run II Collaboration] Phys. Rev. D 75, $092004(2007)$

"Inclusive search for new physics with like-sign dilepton events in $p \bar{p}$ collisions at $\sqrt{s}=1.96-\mathrm{TeV} "$ A. Abulencia et al. [CDF Collaboration] Phys. Rev. Lett. 98, 221803 (2007) [arXiv:hep-ex/0702051]

"Search for anomalous production of multi-lepton events in $p \bar{p}$ collisions at $\sqrt{s}=1.96-\mathrm{TeV} "$ A. Abulencia et al. [CDF Collaboration] Phys. Rev. Lett. 98, 131804 (2007) [arXiv:0706.4448 [hep-ex]]

"Measurement of the Inclusive Jet Cross Section using the $\boldsymbol{k}_{\mathrm{T}}$ algorithm in $p \bar{p}$ Collisions at $\sqrt{s}=1.96 \mathrm{TeV}$ with the CDF II Detector" A. Abulencia et al. [CDF - Run II Collaboration] Phys. Rev. D 75, 092006 (2007) [Erratum-ibid. D 75, 119901 (2007)] [arXiv:hep-ex/0701051]

"Measurement of the Helicity Fractions of W Bosons from Top Quark Decays using Fully Reconstructed $\boldsymbol{t} \overline{\boldsymbol{t}}$ Events with CDF II" A. Abulencia et al. [CDF II Collaboration] Phys. Rev. D 75, 052001 (2007) [arXiv:hep-ex/0612011]

"Measurement of the $\mathrm{B}+$ production cross section in $\mathrm{p}$ anti-p collisions at $\mathrm{s}^{* *}(1 / 2)=1960-\mathrm{GeV}^{\prime}$ A. Abulencia et al. [CDF Collaboration] Phys. Rev. D 75, 012010 (2007) [arXiv:hep-ex/0612015]

"Measurement of the top-quark mass in all-hadronic decays in p anti-p collisions at CDF II" T. Aaltonen et al. [CDF Run II Collaboration] Phys. Rev. Lett. 98, 142001 (2007) [arXiv:hep-ex/0612026]

"Analysis of the quantum numbers $\mathbf{J}(\mathrm{PC})$ of the $\mathrm{X}(3872)$ " A. Abulencia et al. [CDF Collaboration] Phys. Rev. Lett. 98, 132002 (2007) [arXiv:hep-ex/0612053]

"Cross section measurements of high-p(T) dilepton final-state processes using a global fitting method" A. Abulencia et al. [CDF Collaboration] Phys. Rev. D 78, 012003 (2008) [arXiv:hep-ex/0612058] 
"Precision measurement of the top quark mass from dilepton events at CDF II" A. Abulencia et al. [CDF - Run II Collaboration] Phys. Rev. D 75, 031105 (2007) [arXiv:hep-ex/0612060]

"Measurement of the Top Quark Mass in $p \bar{p}$ Collisions at $\sqrt{s}=1.96 \mathrm{TeV}$ using the Decay Length Technique" A. Abulencia et al. [CDF - Run II Collaboration] Phys. Rev. D 75, 071102 (2007) [arXiv:hep-ex/0612061]

"Search for Exotic $S=-2$ Baryons in $p \bar{p}$ Collisions at $\sqrt{s}=1.96 \mathrm{TeV}$ " A. Abulencia et al. [CDF Collaboration] Phys. Rev. D 75, 032003 (2007) [arXiv:hepex/0612066]

"Search for W' boson decaying to electron-neutrino pairs in $\mathrm{p}$ anti-p collisions at $\mathrm{s}^{* *}(1 / 2)=1.96-\mathrm{TeV} " \mathrm{~A}$. Abulencia et al. [CDF Collaboration] Phys. Rev. D 75, 091101 (2007) [arXiv:hep-ex/0611022]

"Observation of exclusive electron positron production in hadron hadron collisions" A. Abulencia et al. [CDF Collaboration] Phys. Rev. Lett. 98, 112001 (2007) [arXiv:hep-ex/0611040]

"Measurement of the ratios of branching fractions $\mathrm{B}\left(B_{s}^{0} \rightarrow D_{s}^{-} \pi^{+} \pi^{+}\right.$ $\left.\pi^{-}\right) / \mathbf{B}\left(B^{0} \rightarrow D^{-} \pi^{+} \pi^{+} \pi^{-}\right)$and $\mathbf{B}\left(B_{s}^{0} \rightarrow D_{s}^{-} \pi^{+}\right) / \mathbf{B}\left(B^{0} \rightarrow D^{-} \pi^{+}\right)$" A. Abulencia et al. [CDF Collaboration] Phys. Rev. Lett. 98, 061802 (2007) [arXiv:hepex/0610045]

"Measurement of the $\Lambda_{b}^{0}$ lifetime in $\Lambda_{b}^{0} \rightarrow J / \Psi \Lambda^{0}$ in p anti-p collisions at $\mathrm{s}^{* *}(1 / 2)=1.96-\mathrm{TeV} "$ A. Abulencia et al. [CDF Collaboration] Phys. Rev. Lett. 98, 122001 (2007) [arXiv:hep-ex/0609021]

"Observation of $B_{s}^{0}$ anti- $B_{s}^{0}$ oscillations" A. Abulencia et al. [CDF Collaboration] Phys. Rev. Lett. 97, 242003 (2006) [arXiv:hep-ex/0609040]

"Search for $\mathrm{V}+\mathrm{A}$ current in top quark decay in $\mathrm{p}$ anti-p collisions at $\mathrm{s}^{* *}(\mathbf{1} / \mathbf{2})=1.96-\mathrm{TeV}$ " A. Abulencia et al. [CDF Collaboration] Phys. Rev. Lett. 98, 072001 (2007) [arXiv:hep-ex/0608062]

"Measurement of the $t \bar{t}$ Production Cross Section in $p \bar{p}$ collisions at $\sqrt{s}=$ 1.96-TeV in the All Hadronic Decay Mode" A. Abulencia et al. [CDF - Run II Collaboration] Phys. Rev. D 74, 072005 (2006) [arXiv:hep-ex/0607095] 


\section{FIELDS OF STUDY}

Major Field: Physics 


\section{TABLE OF CONTENTS}

Page

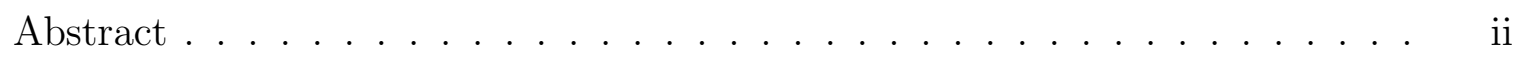

Acknowledgments ........................ iii

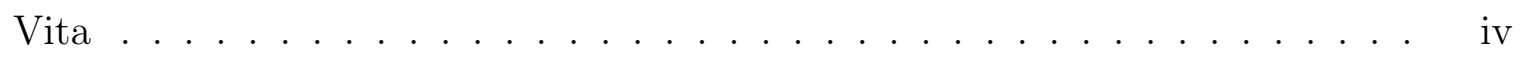

List of Tables . . . . . . . . . . . . . . . . . . xv

List of Figures $\ldots \ldots \ldots \ldots \ldots \ldots \ldots \ldots \ldots$ xvii

Chapters:

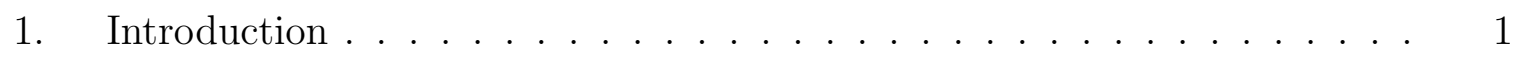

2. The Standard Model and The Higgs Mechanism . . . . . . . . . . . . 4

2.1 The Standard Model . . . . . . . . . . . . . . . . . . . . . 4

2.2 Motivation for Symmetry Breaking . . . . . . . . . . . 7

2.3 Higgs Mechanism, Goldstone's Theorem, and the Higgs Boson . . . 9

2.4 Experimental Limits and Theoretical Constraints . . . . . . . . . 13

2.4.1 Direct Search at LEP . . . . . . . . . . . . . . . . . 13

2.4 .2 Indirect Constraints . . . . . . . . . . . . . . . . 14

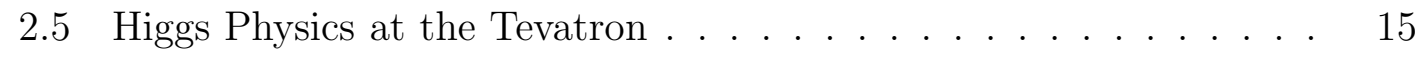

2.5.1 Higgs Boson Production . . . . . . . . . . . . . 15

2.5 .2 Higgs Boson Decay . . . . . . . . . . . . . . . . . 16

$2.5 .3 W H$ Production and Decay $\ldots \ldots \ldots \ldots$ 
3. Experimental Apparatus . . . . . . . . . . . . . . . . . . . . 22

3.1 The Tevatron and the Accelerator Chain . . . . . . . . . . . 22

3.1 .1 Proton Acceleration . . . . . . . . . . . . . . . 22

3.1.2 Anti-proton Production and Acceleration . . . . . . . . 24

3.1 .3 Tevatron . . . . . . . . . . . . . . . . . 25

3.1 .4 Luminosity . . . . . . . . . . . . . . . . 25

3.2 The Collider Detector at Fermilab _ . . . . . . . . . . 26

3.2 .1 Particle-Detector Interactions . . . . . . . . . . . . 27

3.2 .2 CDF Coordinate System . . . . . . . . . . . . . . . . 27

3.2 .3 Cherenkov Luminosity Counter . . . . . . . . . . . . . . . 29

3.2 .4 Solenoid . . . . . . . . . . . . . . . . . . . 30

3.2 .5 Silicon Tracking . . . . . . . . . . . . . . . 30

3.2 .6 Central Outer Tracker . . . . . . . . . . . . . . . . . 31

3.2 .7 Calorimeters . . . . . . . . . . . . . . . . . . . . 33

3.2 .8 Muon Detectors . . . . . . . . . . . . . . . . . . . 34

3.3 Data Acquisition . . . . . . . . . . . . . . . . . . . . 35

4. Object Reconstruction . . . . . . . . . . . . . . . . . . . . . 39

4.1 Electron Identification . . . . . . . . . . . . . . . . . . . . . . 39

4.2 Muon Identification . . . . . . . . . . . . . . . . . . 40

4.3 Isolated Track Identification . . . . . . . . . . . . . . . . . 40

4.3 .1 Isolated Track Scale factor . . . . . . . . . . . . . . . . 44

4.4 Jet Identification . . . . . . . . . . . . . . . . . . . . . . . 46

4.5 Missing Transverse Energy Reconstruction . . . . . . . . . . . . 47

4.6 B-jet Identification Algorithms . . . . . . . . . . . . . . . . . . . 48

4.6.1 Secondary vertex $b$-Tagging . . . . . . . . . . . . . 49

4.6 .2 Jet Probability $b$-tagging . . . . . . . . . . . . . 50

5. Event Selection and Dataset . . . . . . . . . . . . . . . . 56

5.1 Trigger Efficiencies . . . . . . . . . . . . . . . . . 56

5.2 Event Selection Requirements . . . . . . . . . . . . . . 58

6. Background Modeling . . . . . . . . . . . . . . . . . . 61

6.1 Non- $W$ QCD Multi-jet . . . . . . . . . . . . . . . . . . 63

6.1.1 QCD Fraction in the Isolated Track Sample . . . . . . . 67

6.2 Mistagged Jets . . . . . . . . . . . . . . . . . . . . . . . . 69

$6.3 W+$ Heavy Flavor . . . . . . . . . . . . . . . . . . 70

6.4 Top and Electroweak Backgrounds . . . . . . . . . . . . 73 
6.5 Summary of Background Estimate . . . . . . . . . . . . . 73

7. Higgs Boson Signal Acceptance . . . . . . . . . . . . . . . . . . . . 81

8. Modeling and Control Regions . . . . . . . . . . . . . . 85

8.1 Pretag Control Plots . . . . . . . . . . . . . . . . 85

8.2 One Tag Plots . . . . . . . . . . . . . . . . . . . . . . 86

8.3 Two SECVTX Tag Plots . . . . . . . . . . . . 93

9. Neural Network Discriminant . . . . . . . . . . . . . . . . . . . . 97

9.1 Artificial Neural Network Overview . . . . . . . . . . . . . . . . . . 97

9.2 Neural Network Description . . . . . . . . . . . . . . . . . . 98

9.3 Neural Network Output . . . . . . . . . . . . . . . . . . 100

10. Results . . . . . . . . . . . . . . . . . . . . 110

10.1 Binned Likelihood Technique . . . . . . . . . . . . . . . . . . . . 110

10.2 Limit on Higgs Boson Production Rate . . . . . . . . . . . . . . . . 112

11. Discussion and Conclusion . . . . . . . . . . . . . . . . . 115

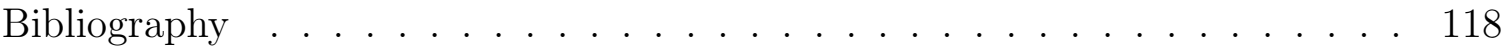




\section{LIST OF TABLES}

Table

Page

2.1 The three generations of quarks and leptons . . . . . . . . . . . 5

2.2 Properties of gauge bosons in the Standard Model . . . . . . . . . . 6

3.1 Tevatron Parameters .................. 26

4.1 Requirements for identifying electron candidates. . . . . . . . . . . . 40

4.2 Muon selection criteria. . . . . . . . . . . . . . . . 41

4.3 Isolated track identification requirements. . . . . . . . . . . . . . . 42

4.4 Requirements for tracks included in Isolation Calculation . . . . . . . 42

5.1 High $p_{T}$ lepton trigger efficiencies. . . . . . . . . . . . . . . . 56

5.2 QCD veto cuts for each lepton category. These cuts are applied to events with exactly one identified b-jet. . . . . . . . . . . 60

6.1 QCD Fractions for various lepton and tag categories . . . . . . . . 65

6.2 The heavy-flavor fractions, given in percent, for the $W+$ jets sample. The results from ALPGEN Monte Carlo have been scaled by the dataderived calibration factor of $1.4 \pm 0.4 \ldots \ldots$. . . . . . . . . 71

6.3 Tagging efficiencies for jets matched to heavy flavor . . . . . . . . . 72

6.4 Theoretical cross sections and uncertainties for the electroweak and top backgrounds. Top cross sections assume a mass of $m_{t}=175 \mathrm{GeV} / c^{2}$. 
6.5 Background summary table for central leptons with one secondary vertex tag. The hashed region indicates the uncertainty on the background estimate. $\ldots \ldots \ldots \ldots \ldots . \ldots \ldots$

6.6 Background summary table for central leptons with one secondary vertex tag and one jet probability tag. The hashed region indicates the uncertainty on the background estimate. . . . . . . . . . .

6.7 Background summary table for central leptons with two secondary vertex tags. The hashed region indicates the uncertainty on the background estimate. . . . . . . . . . . . . . .

6.8 Background summary table for isolated tracks with one secondary vertex tag. The hashed region indicates the uncertainty on the background estimate.

6.9 Background summary table for isolated tracks with one secondary vertex tag and one jet probability tag. The hashed region indicates the uncertainty on the background estimate. . . . . . . . . . .

6.10 Background summary table for isolated track events with two secondary vertex tags. The hashed region indicates the uncertainty on the background estimate. . . . . . . . . . . . . . .

7.1 Expected number of $\mathrm{WH}$ events at a $\mathrm{M}(\mathrm{H})=120 \mathrm{GeV} / \mathrm{c}^{2}$, shown broken down for tag categories and lepton types $\ldots \ldots \ldots \ldots$. . . . .

7.2 Systematic uncertainty on the $W H$ acceptance. "ST+ST" refers to double secondary vertex tagged events while "ST+JP" refers to secondary vertex plus jet probability tagged events. . . . . . . . . .

10.1 Expected and observed limits as a function of Higgs mass for the combined search of Tight Lepton and Isotrk events, including all tag categories. The Higgs masses are in units of $\mathrm{GeV} / \mathrm{c}^{2}$, and the limits are expressed as ratio of experimental limit to the theoretical production rate. . . . . . . . . . . . . . . . . . . 


\section{LIST OF FIGURES}

Figure

Page

2.1 The potential for $\mu^{2}>0$ (left) and $\mu^{2}<0$ for a real scalar $\phi$. . . . 10

2.2 ZH Production at the LEP $e^{+} e^{-}$collider. . . . . . . . . . . . . . . 14

2.3 Constraints of precision electroweak measurements on the Higgs mass, plotted a function of $M_{t}$ and $M_{W}$ [1]. The dashed circle represents indirect constraints on $M_{t}$ and $M_{W}$. The solid circle represents current direct measurements of $M_{t}$ and $M_{W}$. The circles show good agreement between indirect constraints and observations. The overlap of the solid circle and the $M_{H}$ lines indicates the region of $M_{H}$ preferred by indirect constraints. . . . . . . . . . . . . . . . .

2.4 The figure shows the quality of fit to precision electroweak data versus Higgs mass. The yellow shaded region shows the exclusion by LEP's direct Higgs search. The solid dark blue curve is the nominal fit, and the light blue band represents theoretical uncertainties on the fit. The $68 \%$ confidence band is at $\Delta \chi^{2}=1$, the $95 \%$ confidence band is at $\Delta \chi^{2}=2.7$ [1]. The alternate dashed and dotted curves represent the different fit results obtained with modified input parameters, such as different theoretical calculations of the vacuum polarization $\left(\Delta \alpha_{h a d}^{(5)}\right)$, and values of $M_{W}$ obtained with low $Q^{2}$ experiments. . . . . . .

2.5 The figure illustrates methods of producing a Higgs boson $(\phi)$ in a hadron collider. Clockwise from the top left, the figures show: production in association with a gauge boson $(W H, Z H)$, direction production through gluon fusion $(g g \rightarrow H)$, production in association with top pairs $(t \bar{t} H)$, and vector boson fusion (VBF). Only $g g H$ and $W H, Z H$ have large cross sections at the Tevatron. . . . . . . . . . . .

2.6 The expected production cross sections of Higgs boson events in different production channels at the Tevatron. . . . . . . . . . . . . 
2.7 Higgs branching ratios vs. Higgs mass. The two dominant branching ratios are $H \rightarrow \bar{b} b$ and $H \rightarrow W^{+} W^{-}$. Note the log scale on the vertical axis. . . . . . . . . . . . . . . . .

2.8 $W H$ Production and decay $(W H \rightarrow \ell \nu b \bar{b})$. This decay chain occurs most often for Higgs boson masses less than $135 \mathrm{GeV} / \mathrm{c}^{2}$. . . . . . . 21

3.1 Tevatron accelerator chain $\ldots \ldots \ldots \ldots \ldots$

3.2 CDF Integrated and Instantaneous Luminosity . . . . . . . . . 27

3.3 Side-view schematic of the CDF detector. The figure shows half a cross sectional view. The detector is forward-backward symmetric and azimuthally symmetric. . . . . . . . . . . . . . . . . . . 28

3.4 A qualitative description of particle interaction with the CDF detector. 29

3.5 The CDF Run II silicon detectors . . . . . . . . . . . . . . 31

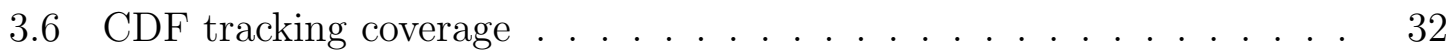

3.7 An illustration of the electromagnetic and hadronic calorimetry in CDF. 34

3.8 Details of the CDF muon detectors $\ldots \ldots \ldots \ldots$

3.9 Graphical representation of the flow of CDF data through the trigger and data acquisition system. . . . . . . . . . . . . 37

4.1 (Left) Angular distribution of muon triggered events. Note the gaps in between the CMUP and CMX acceptance. (Right) Isolated track events fill in the gaps in the muon trigger coverage. The analysis uses both lepton triggered and isolated track events. . . . . . . . . . . 43

4.2 Isolated track reconstruction scale factor vs. lepton $\phi \ldots \ldots \ldots \ldots$

4.3 Isolated track reconstruction scale factor vs. $\eta$. . . . . . . . 47

4.4 Isolated track reconstruction scale factor vs. $p_{T} \ldots \ldots \ldots \ldots$

4.5 SECvTX tag efficiency vs. $E_{T} \ldots \ldots \ldots \ldots \ldots \ldots \ldots$

xviii 
4.6 SECVTX mistag rate vs. $E_{T} \ldots \ldots \ldots \ldots 2$

4.7 Track impact parameters from primary and secondary vertices. . . . .

4.8 Distribution of track impact parameters from primary and secondary vertices. . . . . . . . . . . . . . . . .

$5.1 \quad E_{T}$ plus jets trigger turn-on curve parameterized as a function of vertex

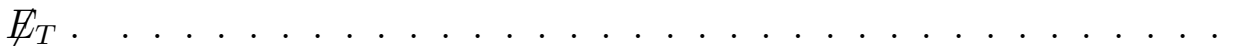

6.1 QCD fraction estimate for pretag CEM, CMUP, events with two jets. The QCD fraction is shown above the plot. . . . . . . . . . . .

6.2 QCD fraction estimate for pretag CMX, ISOTRK events with two jets. The QCD fraction is shown above the plot. . . . . . . . . . . .

6.3 QCD fraction estimate for pretag CEM, ISOTRK events with no kinematic QCD vetos applied. The QCD fractions in the regions above $E_{T}>20 \mathrm{GeV}$ for the two samples are comparable. . . . . . . . .

6.4 Number of expected and observed background events for events with exactly one SECVTX tag, shown as a function of jet multiplicity. The plots show tight leptons (left) and isolated tracks (right). . . . . . .

6.5 Number of expected and observed background events for events with one SECVTX tag and one Jet Probability tag, shown as a function of jet multiplicity. The plots show tight leptons (left) and isolated tracks (right) . . . . . . . . . . . . . . . . .

6.6 Number of expected and observed background events for events with one SECVTX tag and one Jet Probability tag, shown as a function of jet multiplicity. The plots show tight leptons (left) and isolated tracks

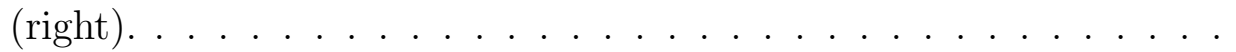

8.1 Distributions of the lead jet $E_{T}$ and second jet $E_{T}$. for pretag isolated track events. The plots show the $W H$ signal at 200 times it's Standard Model expectation. . . . . . . . . . . . . . . 
8.2 Distributions of the $\#_{T}$ and lepton $p_{T}$ for pretag isolated track events. The plots show the $W H$ signal at 200 times it's Standard Model expectation. . . . . . . . . . . . . . . .

8.3 Distributions of $M_{T}(W)$ and $M(j j+)$ for pretag isolated track events. The plots show the $W H$ signal at 200 times it's Standard Model ex-

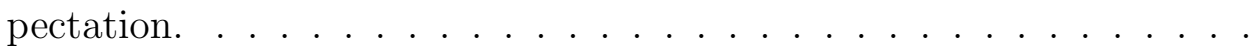

8.4 Distributions of the lead jet $E_{T}$ and second jet $E_{T}$ for one tag isolated track events. The plots show the $W H$ signal at 50 times it's Standard Model expectation. . . . . . . . . . . . . . . . . .

8.5 Distributions of the $\not_{T}$ and lepton $p_{T}$ for one tag isolated track events. The plots show the $W H$ signal at 50 times it's Standard Model expectation. . . . . . . . . . . . . . . . .

8.6 Distributions of the $M_{T}$ for one tag isolated track events. The plots show the $W H$ signal at 50 times it's Standard Model expectation. . .

8.7 Distributions of the lead jet $E_{T}$ and second jet $E_{T}$, for two SECVTX tag isolated track events. The plots show the $W H$ signal at 50 times it's Standard Model expectation.

8.8 Distributions of the $E_{T}$ and lepton $p_{T}$ for two SECVTX tag isolated track events. The plots show the $W H$ signal at 50 times it's Standard Model expectation.

8.9 Distributions of the $M_{T}$ for two SECVTx tag isolated track events. The plots show the $W H$ signal at 50 times it's Standard Model expectation. 96

9.1 Neural Network input distributions for isolated track $W+2$ jets events in the pretag control region. The distributions shown are $M_{j j+}$ (left), $\sum E_{T}$ (Loose Jets) (left).

9.2 Neural Network input distributions in isolated track pretag $W+2$ jet events. The distributions shown are $p_{T}$ Imbalance (left) and $M_{l \nu j}^{\min }$ (right).

9.3 Neural Network input distributions in isolated track pretag $W+2$ jet events. The distributions shown are $\Delta R$ (lepton- $\left.\nu_{\max }\right)$ (left) and $P_{T}(W+H)$ (right). . . . . . . . . . . . . . . . . 103 
9.4 Neural Network input distributions for isolated track one tag $W+2$ jets events. The distributions shown are $M_{j j+}$ (left), $\sum E_{T}$ (Loose Jets) (left). . . . . . . . . . . . . . . . . . . 104

9.5 Neural Network input distributions in isolated track one tag $W+2$ jet events. The distributions shown are $p_{T}$ Imbalance (left) and $M_{l \nu j}^{\min }$

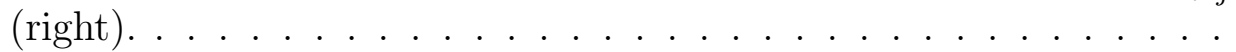

9.6 Neural Network input distributions in isolated track one tag $W+2$ jet events. The distributions shown are $\Delta R$ (lepton- $\left.\nu_{\max }\right)$ (left) and $P_{T}(W+H)$ (right). . . . . . . . . . . . . . . . . 106

9.7 Neural Network output distributions for events with one secvtx tag. The plots show isolated track events (left) and lepton triggered events (right). . . . . . . . . . . . . . . . 107

9.8 Neural Network output distributions for events with one secvtx tag. The plots show isolated track events (left) and lepton triggered events (right). . . . . . . . . . . . . . . . . . . . . . 108

9.9 Neural Network output distributions for events with one secvtx tag. The plots show isolated track events (left) and lepton triggered events

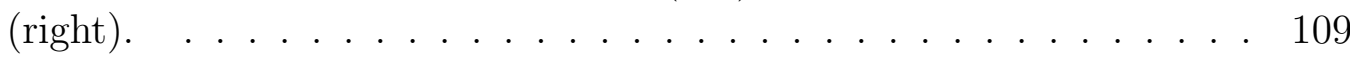

$10.195 \%$ confidence level upper limit on $W H$ production. . . . . . . . . . 113

11.1 CDF 95\% confidence level upper limit for various Higgs production channels. . . . . . . . . . . . . . . . 116 


\section{CHAPTER 1}

\section{INTRODUCTION}

The Standard Model a highly successful explanation of the universe in terms of its fundamental constituents. It explains a wide variety of phenomena, including the spectrum of the hydrogen atom and the process of radioactive decay. The Standard Model is a description of fundamental particles and the interactions between them expressed in the language of symmetries. Its description of particles and interactions has been tested and validated across a wide range of energies in numerous experiments. It is an extremely successful model of the universe.

Tests of the Standard Model have been numerous, but they have not been exhaustive. There are still exciting new measurements to be made which could expand our fundamental knowledge. One of the most exciting unanswered questions is, "How do fundamental particles acquire mass?". While we understand the mass of composite objects, such as the proton, the mass of fundamental particles remains untested.

The Standard Model offers a potential explanation for the origin of mass. The explanation is based in the resolution of two conflicting constraints. On one hand, we want particles to obey the symmetries and conservation laws that govern nature. In order to obey the conservation laws the particles must be massless. On the other hand, we know experimentally that particles have mass. Both constraints can be satisfied 
at the same time if mass is not an intrinsic property, but rather a consequence of new interaction. The new interaction provides mass in a way that hides or "breaks" the symmetries without removing them entirely. Dynamic mass generation and symmetry breaking predicts the existence of a new particle, the Higgs boson. The Standard Model predicts that Higgs boson production will be extremely rare. The rarity of Higgs production and the Higgs decay properties depend on the Higgs mass. There is no prediction for the Higgs mass, and theoretical predictions provide a broad range of possible Higgs masses. Prior searches found no evidence for Higgs production for a range of Higgs masses (less than $115 \mathrm{GeV} / \mathrm{c}^{2}$ ), but many viable Higgs masses remain to be tested.

This dissertation explores the origin of mass with a new direct search for the Higgs. Chapter 2 briefly introduces Higgs theory and summarizes prior direct and indirect searches. The prior search results guide the new search to the frontier of high energy physics.

The current high-energy frontier is at the Tevatron proton-antiproton collider in Batavia, Illinois. Our search uses Tevatron collisions recorded by the Collider Detector at Fermilab (CDF) experiment between February 2002 and April 2008. Chapter 3 describes the Tevatron and the CDF Detector.

We analyze each collision, or event, for evidence of the Higgs. Chapter 5 describes the how the data is collected and the methodology for selecting a subset of the data likely to contain Higgs events. The search sample will also include events from other known processes, collectively referred to as backgrounds. We estimate the composition of the background in Chapter 6 and calculate the expected number of Higgs 
events in Chapter 7. We demonstrate validity of our background model in Chapter 8 by looking at event kinematics in regions with small amounts of signal contamination.

Assuming a Higgs mass in the range 115 to $130 \mathrm{GeV} / \mathrm{c}^{2}$, Higgs events make up a small fraction of the total search sample, so it is important to consider as many features of each event as possible in order to reject more copious background processes. Our search uses an Artificial Neural Network (ANN) to maximize the amount of discriminating information. Chapter 9 describes the neural network developed to discriminate Higgs events. The network combines many kinematic variables into a single distribution optimized to separate Higgs events from backgrounds.

We examine the Neural Network output and find no evidence for an excess of Higgs signal events above the background. Chapter 10 describes the methodology used to search for an excess and an set upper limit on the amount of Higgs events that could have been in the sample. Chapter 11 discusses the interpretation of the result. 


\section{CHAPTER 2}

\section{THE STANDARD MODEL AND THE HIGGS MECHANISM}

The following chapter gives an overview of the Standard Model. It focuses on the particles in the model, the Higgs mechanism, and results of both direct and indirect searches for the Higgs boson.

\subsection{The Standard Model}

The particles in the Standard Model can be classified as either fermions or bosons. Two fermions cannot share the same quantum state, but bosons have no rules limiting state occupancy. Fermions can be thought of as the building blocks that compose matter, and bosons the adhesive that transmits the forces that hold the blocks together.

Fermions are divided into quarks and leptons. Quarks are different from leptons because they have "color", a unit of charge for the strong force. Both categories can be organized according to their electroweak quantum numbers, as shown in table 2.1. Each pair in parentheses in the table represents an electroweak doublet. There are three generations of doublets, each with identical electroweak quantum numbers and different masses. The generations have been arranged in order of increasing mass. 
There are large mass differences across generations. The up quark, for instance, has a mass of approximately 1.5 to $3.3 \mathrm{MeV}$, while the top quark has a mass of approximately $171 \mathrm{GeV}[2]^{1}$. The heavier generations of matter quickly decay into lighter generations. It is the lightest generation that makes up common forms of matter. The nucleus consists of protons and neutrons, which are bound states of up and down quarks. Electrons bind to the nucleus to form atoms. Neutrinos are released from the nucleus during $\beta$ decay.

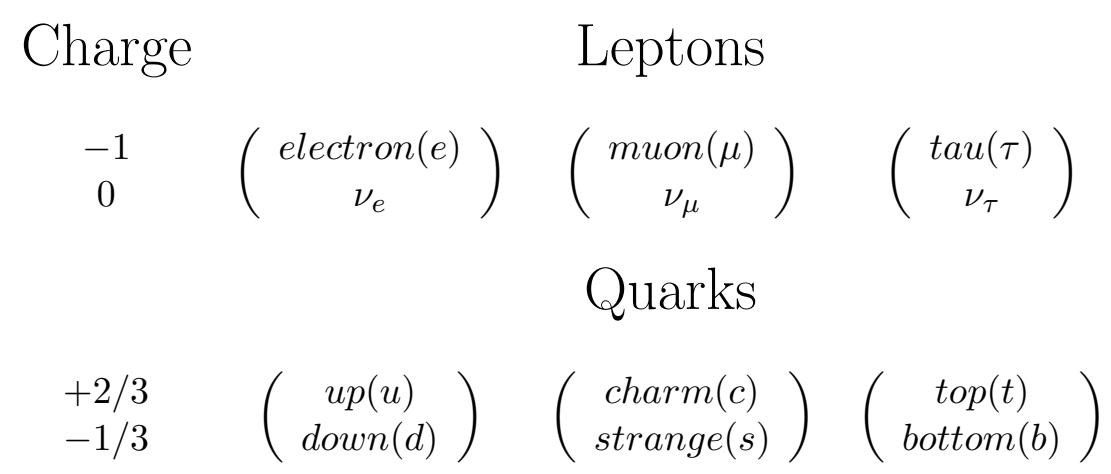

Table 2.1: The three generations of quarks and leptons

Table 2.2 shows the gauge bosons of the Standard Model. The gauge bosons are classified according to the force they mediate. The massive $\mathrm{W}$ and $\mathrm{Z}$ bosons mediate the Weak Force. The massless gluon and photon mediate the Strong and Electromagnetic Force, respectively. The Strong force is responsible for binding quarks together inside protons and neutrons, and binding protons within a nucleus. The Weak Force is less powerful than the strong force and, unlike the strong force, it does not conserve quark numbers. The violation of quark number makes the Weak Force responsible for

\footnotetext{
${ }^{1}$ The up quark is only found in bound states with at least one other quark, so its mass is difficult to express exactly. The value given here is the value agreed upon in reference [2]. The top quark does not share the problem because it decays before it can form bound states.
} 
the decay of the heavy generations into the light generations. The Electromagnetic Force is the weakest of the three forces in the Standard Model and it is responsible for many familiar phenomena. Its roles include binding electrons to nuclei and transmitting energy as light.

Experiments have directly observed all of the quarks, leptons, and gauge bosons. The Higgs boson is the only particle that has eluded experimental searches. It is not a gauge boson and does not mediate a force. It plays an role in giving mass to the $W, Z$, and fermions. It is not straightforward to give mass to fundamental particles. We need to look at the details of the Standard Model's description of particles and forces to see the role of the Higgs boson.

$\begin{array}{ccc}\text { Force } & \text { Bosons } & \text { Mass } \\ \text { Electromagnetic } & \text { photon }(\gamma) & 0 \frac{G e V}{c^{2}} \\ \text { Strong } & \text { gluons }(g) & 0 \frac{G e V}{c^{2}} \\ \text { Weak } & W^{ \pm} & 80.4 \frac{G e V}{c^{2}} \\ & Z^{0} & 91.2 \frac{G e V}{c^{2}}\end{array}$

Table 2.2: Properties of gauge bosons in the Standard Model

The particles and their interactions can be concisely described using group theory, the mathematics of symmetry. Noether's theorem provides the fundamental connection between symmetries and a description of the world. The theorem states that there is a conserved quantity for each symmetry of the Lagrangian. The theorem provides a unified language for discussing seemingly disparate conserved quantities, 
such as charge and momentum. The symmetry groups that describe the Standard Model are:

$$
S U(3)_{\text {color }} \otimes S U(2)_{L} \otimes U(1)_{Y}
$$

$S U(3)_{\text {color }}$ is the gauge group for the strong force. $S U(2)_{L} \otimes U(1)_{Y}$ is the gauge group for both the electromagnetic and weak forces, which can be expressed as a unified electroweak force. We construct our theory by building a Lagrangian that obeys the model's symmetries.

\subsection{Motivation for Symmetry Breaking}

It is not straightforward to develop a consistent theoretical description of the Standard Model's massive particles. The difficulty arises in the inclusion of mass terms in the electroweak Lagrangian. Mass terms have the general form,

$$
\mathcal{L}=m^{2} \bar{\psi} \psi
$$

where $m$ is the particle mass and $\psi$ is the particle field with dimensions of mass. Terms such as 2.2 are difficult to include in Lagrangians because they do not preserve symmetries and therefore spoil conservations laws. We illustrate how mass terms ruin symmetries by trying to add massive vector bosons to Quantum Electrodynamics (QED), and trying to add massive fermions to electroweak interactions.

The QED Lagrangian is

$$
\mathcal{L}=i \bar{\psi}\left(\gamma^{\mu} \delta_{\mu}-m^{2}\right) \bar{\psi}+e\left(\bar{\psi} \gamma^{\mu} A_{\mu} \psi\right)-\frac{1}{4} F_{\mu \nu} F^{\mu \nu}
$$


where $\psi$ is the particle field, $m$ is the particle mass, e is the particle charge, $A_{\mu}$ is the gauge field (electromagnetic field), and $F_{\mu \nu}$ is the field strength, defined as

$$
F_{\mu \nu}=\delta_{\mu} A_{\nu}-\delta_{\nu} A_{\mu}
$$

The Lagrangian 2.3 has been constructed to preserve $\mathrm{U}(1)$ local phase symmetry that corresponds charge conservation. The fields transform under U(1) local phase rotation $\alpha(x)$ in the following way:

$$
\begin{aligned}
\psi & \rightarrow e^{i \alpha(x)} \psi \\
\bar{\psi} & \rightarrow e^{-i \alpha(x)} \psi \\
A_{\mu} & \rightarrow A_{\mu}+\frac{1}{e} \delta \alpha(x)
\end{aligned}
$$

When we apply these transformations to 2.3, we find that it is invariant. If we add a vector boson mass term, we find that it is not invariant under the same phase rotation, which spoils the $\mathrm{U}(1)$ symmetry:

$$
\begin{aligned}
\frac{1}{2} m^{2} A_{\mu} A^{\mu} & \rightarrow \frac{1}{2} m^{2}\left(A_{\mu} A^{\mu}+\frac{1}{e} A_{\mu} \delta^{\mu} \alpha(x)+\frac{1}{e} \delta_{\mu} \alpha A^{\mu}+\frac{1}{e^{2}}\left(\delta_{\mu} \alpha(x)\right)\left(\delta^{\mu} \alpha(x)\right)(2 .\right. \\
& \neq \frac{1}{2} m^{2} A_{\mu} A^{\mu}
\end{aligned}
$$

The Lagrangian 2.3 can have a fermion mass term because it only needs to preserve U(1) symmetry. Fermion mass terms are not possible for other symmetries, such as the electroweak $S U(2)_{L} \otimes U_{Y}(1)$. To see this, consider electrons in the Weyl basis

$$
\psi=\left(\begin{array}{c}
e_{L} \\
e_{R}
\end{array}\right)
$$

Electrons have weak hypercharge $Y\left(e_{L}\right)=-1$ and $Y\left(e_{R}\right)=-2$. Under $U(1)_{Y}$, the fields transform as,

$$
\begin{aligned}
& e_{R} \rightarrow e^{i Y\left(e_{R}\right) / 2 \alpha(x)} \psi_{R}=e^{-i \alpha(x)} \psi_{R} \\
& e_{L} \rightarrow e^{-i Y\left(e_{L}\right) / 2 \alpha(x)} \psi_{L}=e^{i Y\left(e_{L}\right) \alpha(x)} \psi_{L}
\end{aligned}
$$


The electron mass term, which is $\frac{1}{2} m \bar{\psi} \psi$, is not invariant under this symmetry,

$$
\begin{aligned}
\frac{1}{2} m \bar{\psi} \psi & =\frac{1}{2} m\left(\bar{\psi}_{L} \psi_{R}+\bar{\psi}_{R} \psi_{L}\right) \\
& \rightarrow \frac{1}{2} m\left(e^{i \alpha(x) / 2} \bar{\psi}_{L} \psi_{R}+e^{-i \alpha(x) / 2} \bar{\psi}_{R} \psi_{L}\right) \\
& \neq \frac{1}{2} m \bar{\psi} \psi
\end{aligned}
$$

We must consider a delicate way to add boson and fermion mass terms since we cannot add them explicitly.

\subsection{Higgs Mechanism, Goldstone's Theorem, and the Higgs Boson}

The Higgs Mechanism provides a way to add mass terms to the Lagrangian without corrupting its symmetries. The mechanism uses interactions with a new field to create a ground state that "hides" or "breaks" the symmetries of the full theory.

Mass generation through symmetry breaking has complications. Goldstone's theorem dictates that breaking a continuous symmetry will produce massless scalars whose existence is in conflict with experiment. The new bosons are equivalent to additional degrees of freedom in the theory. A careful choice of gauge can show that the extra Goldstone Boson degrees of freedom are synonymous with the longitudinal polarization of the heavy gauge bosons. Vector bosons can be described as "eating up" the Goldstone bosons when they acquire mass.

We will consider an example following reference [3] that illustrates how the Higgs Mechanism can create massive gauge bosons without introducing massless Goldstone bosons. We consider a theory of complex scalars, $\phi$, interacting according to

$$
\mathcal{L}=\left(D_{\mu} \phi\right)^{*}\left(D^{\mu} \phi\right)-\mu^{2} \phi^{*} \phi-\lambda\left(\phi^{*} \phi\right)^{2}
$$




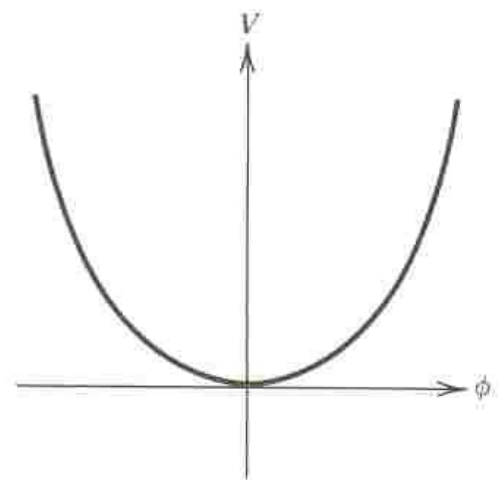

(a)

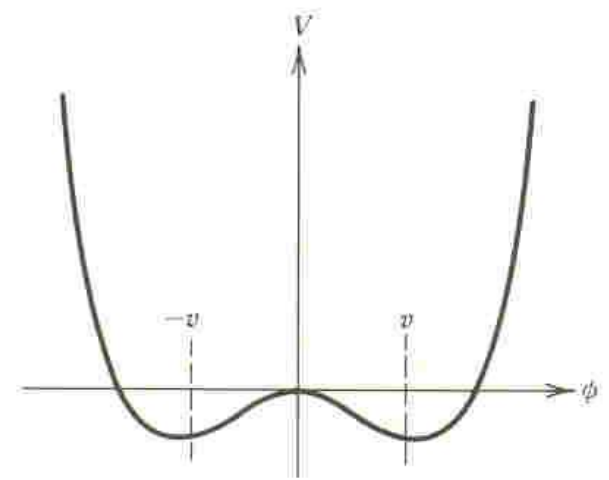

(b)

Figure 2.1: The potential for $\mu^{2}>0$ (left) and $\mu^{2}<0$ for a real scalar $\phi$.

where $D_{\mu}$ is a covariant derivative, $\mu^{2}$ and $\lambda>0$ are free parameters that describe a potential $V(\phi)=\mu^{2} \phi^{*} \phi+\lambda\left(\phi^{*} \phi\right)^{2}$. Figure 2.1 shows the potential $V(\phi)$ for two $\mu^{2}>0$, where there is one minimum, and $\mu^{2}<0$, where there are two minima. The case of one minimum is the case of particle with mass $\mu$. It is qualitatively different from the case of two minima. The two minima of the potential are

$$
\phi= \pm v= \pm \sqrt{-\mu^{2} / \lambda}
$$

The minima of the system become important when we use perturbation theory to calculate the physics of the system. We must choose a stable minimum, or ground state, for our perturbative expansion. Shifting the theory to a non-zero ground state changes the properties of the potential, namely, it has removed the symmetry about the $V(\phi)$ axis $^{2}$. We say the ground state "breaks the symmetry" since it no longer shares the symmetry of the full theory. The choice of ground state is arbitrary, and

${ }^{2}$ The perturbative expansion itself did not change the total physics. If we took our expansion to high enough order, it would still have the symmetries of the full theory. The lower-orders of the ground state have changed. 
we select the ground state $+v$. We expand the complex scalar $\phi$ about $v$,

$$
\phi=\sqrt{1 / 2}(v+h(x)) e^{i \Theta(x) / v}
$$

where $h(x)$ and $\Theta(x)$ are real fields representing perturbations about the ground state, and we have used $i \Theta(x) / v \sim e^{i \Theta(x) / v}$. We have been careful to expand $\phi$ in a gauge that will manifestly not have Goldstone bosons. We transform the gauge fields and derivative in a way that accounts for the $\mathrm{U}(1)$ local phase shift $\alpha(x)$ and the gauge shift that will remove the Goldstone bosons, $\Theta(x)$. The transformations are:

$$
\begin{aligned}
\phi & \rightarrow e^{i \alpha(x)} \phi \\
D_{\mu} & \rightarrow \delta_{\mu}-i e A_{\mu}-i / v \delta_{\mu} \Theta(x) \\
A_{\mu} & \rightarrow A_{\mu}+\frac{1}{e} \delta_{\mu} \alpha(x)+\frac{1}{e v} \delta_{\mu} \Theta(x) .
\end{aligned}
$$

The Lagrangian is now

$$
\mathcal{L}=\frac{1}{2}\left(\delta_{\mu} h\right)^{2}-\lambda v^{2} h^{2}+\frac{1}{2} e^{2} v^{2} A_{\mu}^{2}-\lambda v h^{3}-\frac{1}{4} \lambda h^{4}+\frac{1}{2} e^{e} A_{\mu}^{2} h^{2}+v e^{2} A_{\mu}^{2} h-\frac{1}{4} F_{\mu \nu} F^{\mu \nu} .
$$

We can identify massive particles by looking for terms of the form $m^{2} \phi^{*} \phi$. We find one massive vector boson $A_{\mu}$ and one new massive scalar $h$, with masses:

$$
\begin{aligned}
& m_{A}=e v \\
& m_{h}=\sqrt{2 \lambda v^{2}} .
\end{aligned}
$$

We have seen that perturbative analysis of the Lagrangian about its symmetry-broken ground state reveals mass terms for our gauge bosons and scalars. The Standard Model uses the same technique to generate mass.

Symmetry breaking in the Standard Model is similar to the complex scalar example. The details are provided elsewhere [3]. The result is three massive gauge bosons 
$\left(W^{ \pm}, Z\right)$, a massless photon, a new massive electrically neutral scalar Higgs Boson $(H)$, and a set of massive fermions ${ }^{3}$. The mass terms for the particles are

$$
\begin{aligned}
M_{W} & =\frac{1}{2} v g \\
M_{Z} & =\frac{1}{2} v \sqrt{g^{2}+g^{\prime 2}} \\
M_{f} & =\frac{y_{f} v}{2} \\
M_{h} & =\sqrt{2 v^{2} \lambda}
\end{aligned}
$$

where $v=246 \mathrm{GeV}$ is the vacuum expectation value of the Higgs field, $g\left(g^{\prime}\right)$ is the known strength of the charged (neutral) current interaction, $\lambda$ is a free term in the scalar potential, and $y_{f}$ is the Yukawa coupling of each fermion to the Higgs field. The couplings $y_{f}$ and $\lambda$ are free parameters in the theory that must be fixed by experimental inputs.

The existence of the Higgs boson is a consequence of the Standard Model method of mass generation. Observing the Higgs boson would confirm that symmetry breaking is the origin of mass. We can hunt for the Higgs boson by looking for Higgs boson interactions with other particles. The Standard Model Lagrangian includes interactions that couple the Higgs boson to each of the fermions and gauge bosons,

$$
\mathcal{L}_{\text {HiggsInt }}=\frac{m_{f}}{v} \overline{\psi_{f}} \psi_{f} H+\left|\left(i \delta_{\mu}-\frac{g}{2} \tau \cdot \mathbf{W}_{\mu}-\frac{g^{\prime} Y}{2} B_{\mu}\right) H\right|^{2}
$$

These interactions mean it will be possible to produce the Higgs boson through high energy collisions, and that the Higgs boson will decay into other particles.

\footnotetext{
${ }^{3}$ Neutrinos are assumed to be massless in our theoretical calculations. Recent neutrino experiments have shown that neutrinos do have mass[2], but its origin is not clear.
} 


\subsection{Experimental Limits and Theoretical Constraints}

The properties of the Standard Model Higgs boson strongly depend on its mass. Theoretically calculations alone do not provide strong constraints on the Higgs mass. The tightest constraints come from direct searches and precision measurements of other Standard Model parameters. The results of these prior investigations indicates that the Higgs boson was possibly just beyond the reach of prior experiments and guides our current search at the Tevatron.

\subsubsection{Direct Search at LEP}

The Large Electron Positron Collider (LEP) at CERN collided electrons and positrons at various center-of-mass energies from 1989 until 2000. The experiments L3, OPAL, DELPHI, and ALEPH analyzed the LEP collisions looking for evidence of the Higgs boson. They searched for a Higgs boson produced in association with a Z boson, as shown in Figure 2.2. The associated search channel was sensitive to Higgs masses less than the difference between the beam energy and the Z mass $\left(M_{H}=\sqrt{s}-M_{Z}\right)$. The experiments searched in various $Z$ decay modes, such as $Z \rightarrow l^{+} l^{-}$and $Z \rightarrow \nu \bar{\nu}$, and combined results from their separate datasets to maximize sensitivity. During the final year of LEP collisions the experiments saw a hint of an excess of Higgs signal events near a mass of $115 \mathrm{GeV} / \mathrm{c}^{2}$. Not enough data was taken to improve the statistical significance of the excess. Ultimately, the LEP experiments excluded $M_{H}<114.4 \mathrm{GeV} / \mathrm{c}^{2}[4]$. 


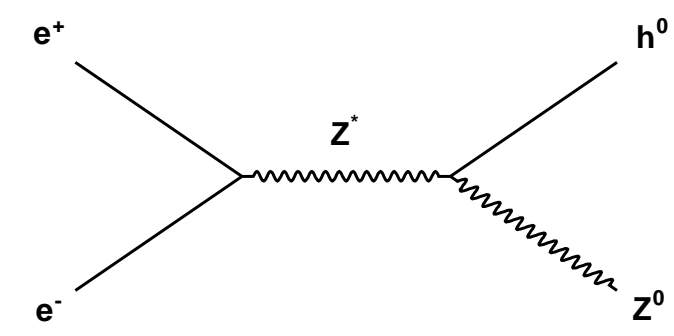

Figure 2.2: ZH Production at the LEP $e^{+} e^{-}$collider.

\subsubsection{Indirect Constraints}

The properties of the Higgs boson can influence the value of other Standard Model parameters through loop diagrams. In particular, radiative corrections from Higgs loops can influence the mass of the $W, Z$, and $t$. Precision measurements of these parameters offer an indirect constraint on possible Higgs masses.

Figures 2.3 and 2.4 summarize the indirect constraints on the Higgs mass. Figure 2.3 shows both a proof-of-concept for the fitting technique and a constraint on $M_{H}$. The agreement of the dashed circle and the solid circle demonstrates that indirect constraints on $M_{t}$ and $M_{W}$ are in good agreement with direct measurements [1]. The figure also shows theoretical predictions of $M_{t}$ and $M_{W}$ for a variety of Higgs mass values. The plot strongly suggests a low Higgs mass because the $M_{t}$ and $M_{W}$ direct measurements only overlap with the low $M_{H}$ lines. Figure 2.4 shows an alternate representation of the constraints. It displays the quality of the Standard Model fit $\left(\Delta \chi^{2}\right)$ as a function of Higgs mass. The preferred fit value is the Higgs mass that minimizes $\Delta \chi^{2}$, with the $68 \%$ and $95 \%$ confidence level values at $\Delta \chi^{2}=1$ and $\Delta \chi^{2}=2.7$, respectively. The one-sided $95 \%$ confidence level upper limit is $M_{H}<154$ 
$\mathrm{GeV} / \mathrm{c}^{2}$. The LEP direct searches rule out the preferred fit value of $84 \mathrm{GeV} / \mathrm{c}^{2}$. If the direct exclusion is included in the limit, the $95 \%$ upper limit increases to $M_{H}<185$ $\mathrm{GeV} / \mathrm{c}^{2}$. If the Standard Model correctly describes the Higgs boson, then the indirect constraints favor a light Higgs boson that was just beyond the reach of the direct LEP search.

Direct searches for the Standard Model Higgs boson are important even if the Standard Model is incomplete. Extensions of the Standard Model, such as supersymmetry (SUSY), can significantly change the Higgs sector [2]. The changes have the potential to alter Higgs production and decay in ways that significantly modify the preferred Higgs mass and potential discovery modes. Direct searches can demonstrate the need for a physics beyond the Standard Model by excluding the Higgs masses that are compatible with indirect constraints.

\subsection{Higgs Physics at the Tevatron}

As of January 2009, the Tevatron is the highest energy accelerator in the world, with collisions at $1.96 \mathrm{TeV}$ center of mass energy. It is currently the only collider capable of producing a Higgs boson. A Higgs boson will immediately decay after it is produced. Its mass determines the most common type of decay products. We will discuss Higgs boson production and decay and focus specifically on low mass Higgs physics.

\subsubsection{Higgs Boson Production}

Hadron colliders can produce Higgs bosons in a variety of ways. Figure 2.5 shows Feynman diagrams for the different potential Higgs boson production mechanisms. The processes with the largest cross sections at the Tevatron are gluon fusion $(g g \rightarrow$ 
$H)$ and gauge boson associated production $(W H, Z H)$. Vector boson fusion and top pair associated production become important processes at larger energies, but have tiny cross sections at the Tevatron. Figure 2.6 shows the Tevatron cross sections for $g g \rightarrow H, W H$, and $Z H$ as a function of Higgs mass. Gluon fusion has the largest cross section at the Tevatron, followed by $W H$ and $Z H$ respectively.

\subsubsection{Higgs Boson Decay}

The Higgs boson can decay into a variety of products. The relative rates of different decay modes depends on the Higgs mass. Figure 2.7 shows the Higgs branching fraction as a function of mass. The dominant decay mode changes at $M_{H}=135$ $G e V / c^{2}$ from $H \rightarrow b \bar{b} \quad$ to $H \rightarrow W^{+} W^{-}$. The change in decay mode is dramatic from an experimental perspective because of the contrast in the decay product's detector signatures. Bottom quarks will hardonize into jets, which are commonly produced in a variety of large cross-section QCD processes. In contrast, the distinct high mass $H \rightarrow W W \rightarrow(e, \mu)+(e, \mu)+2 \nu$ decay mode stands out from QCD background processes and many electroweak backgrounds. Searches for low-mass Higgs boson $\left(M_{H}<135 \mathrm{GeV} / \mathrm{c}^{2}\right)$ decaying to $b \bar{b}$ must reduce the potentially overwhelming backgrounds.

\subsection{3 $W H$ Production and Decay}

The indirect Higgs boson constraints motivate a search for the the light Higgs boson decay signature, $H \rightarrow b \bar{b}$. $W H$ associated production offers a good production channel for a light Higgs boson search. The $W H$ cross section is moderately large, and the $W \rightarrow l, \nu$ in the final state helps to reduce the backgrounds that could overwhelm 
direct light Higgs boson production. Figure 2.8 illustrates $W H$ production and decay to a $\ell \nu b \bar{b}$ final state. Our Higgs boson search will focus on $W H \rightarrow \ell \nu b \bar{b}$ channel. 


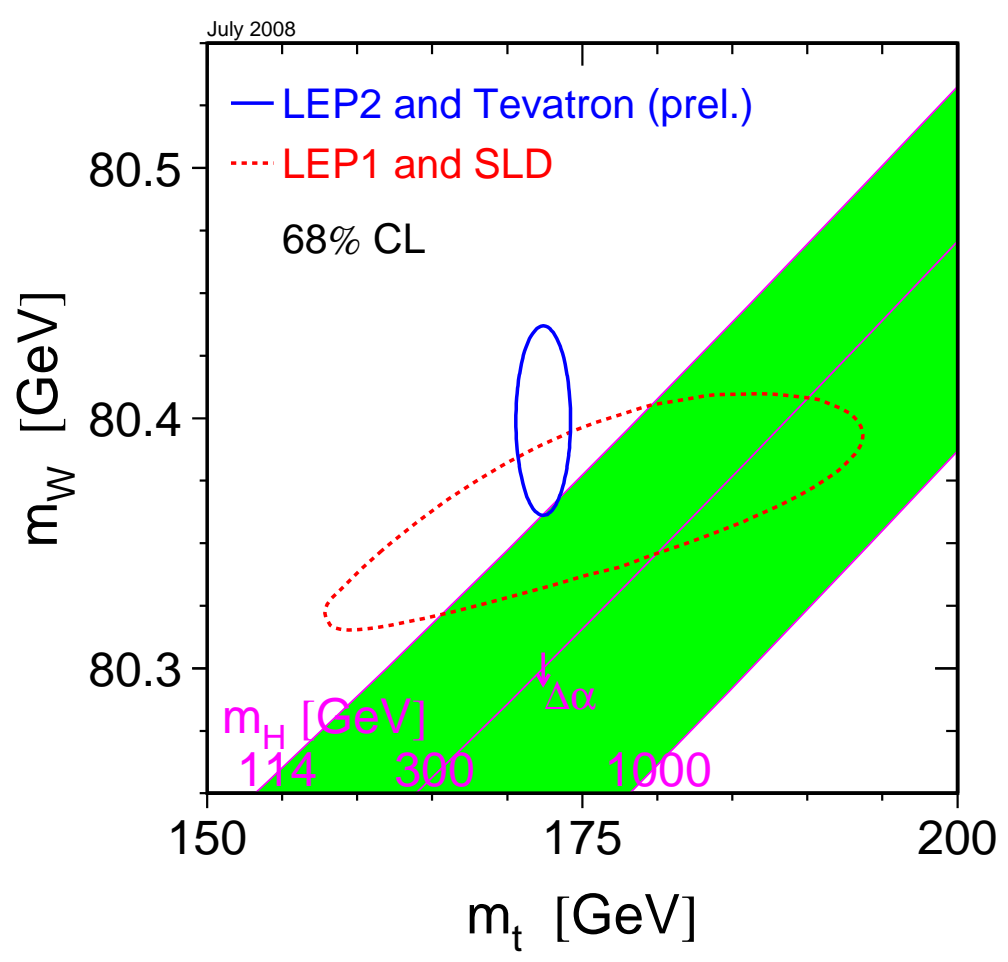

Figure 2.3: Constraints of precision electroweak measurements on the Higgs mass, plotted a function of $M_{t}$ and $M_{W}$ [1]. The dashed circle represents indirect constraints on $M_{t}$ and $M_{W}$. The solid circle represents current direct measurements of $M_{t}$ and $M_{W}$. The circles show good agreement between indirect constraints and observations. The overlap of the solid circle and the $M_{H}$ lines indicates the region of $M_{H}$ preferred by indirect constraints. 


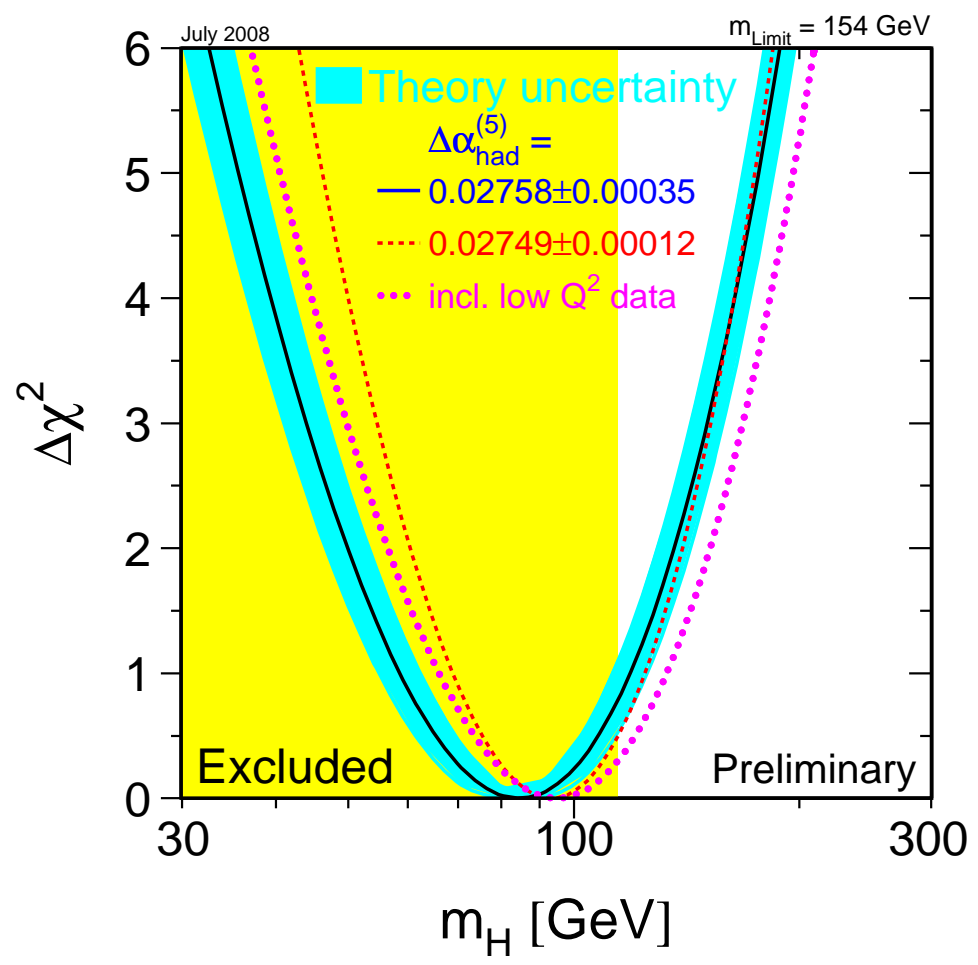

Figure 2.4: The figure shows the quality of fit to precision electroweak data versus Higgs mass. The yellow shaded region shows the exclusion by LEP's direct Higgs search. The solid dark blue curve is the nominal fit, and the light blue band represents theoretical uncertainties on the fit. The $68 \%$ confidence band is at $\Delta \chi^{2}=1$, the $95 \%$ confidence band is at $\Delta \chi^{2}=2.7$ [1]. The alternate dashed and dotted curves represent the different fit results obtained with modified input parameters, such as different theoretical calculations of the vacuum polarization $\left(\Delta \alpha_{\text {had }}^{(5)}\right)$, and values of $M_{W}$ obtained with low $Q^{2}$ experiments. 

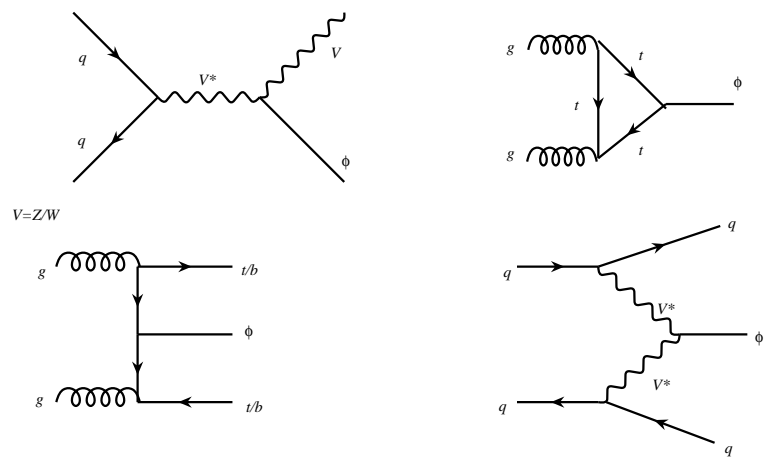

Figure 2.5: The figure illustrates methods of producing a Higgs boson $(\phi)$ in a hadron collider. Clockwise from the top left, the figures show: production in association with a gauge boson $(W H, Z H)$, direction production through gluon fusion $(g g \rightarrow H)$, production in association with top pairs $(t \bar{t} H)$, and vector boson fusion (VBF). Only $g g H$ and $W H, Z H$ have large cross sections at the Tevatron.

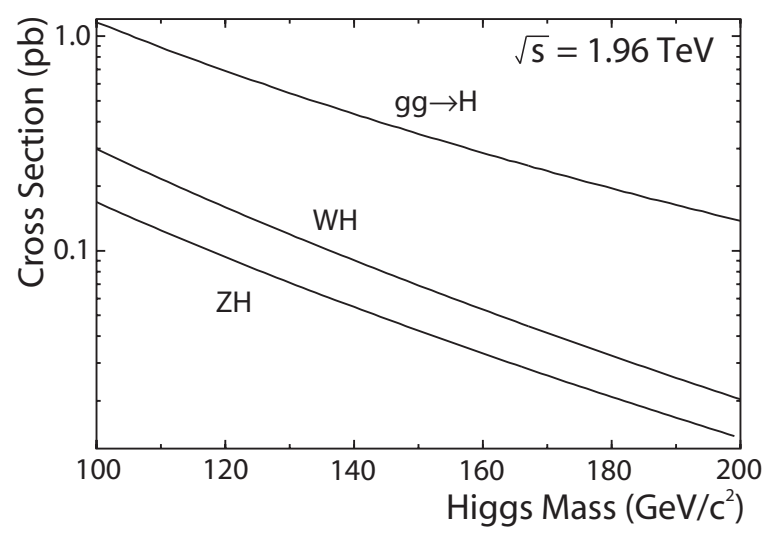

Figure 2.6: The expected production cross sections of Higgs boson events in different production channels at the Tevatron. 


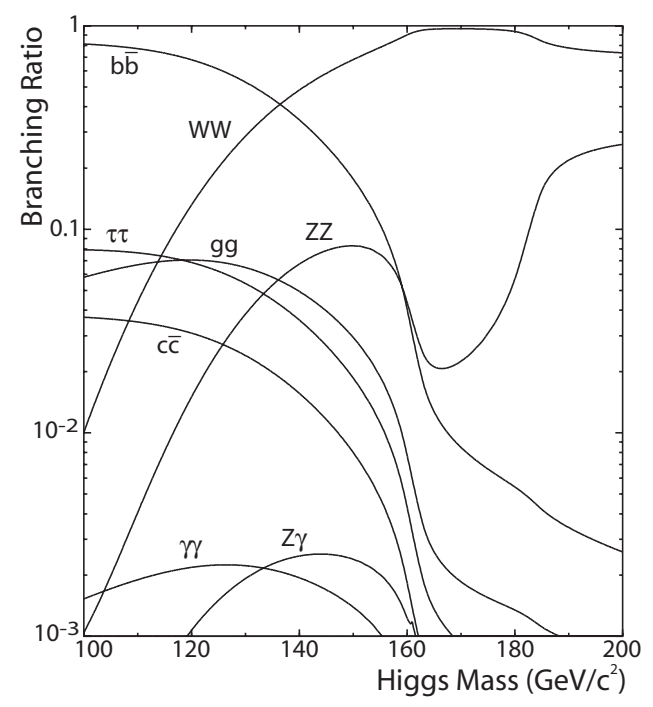

Figure 2.7: Higgs branching ratios vs. Higgs mass. The two dominant branching ratios are $H \rightarrow \bar{b} b$ and $H \rightarrow W^{+} W^{-}$. Note the log scale on the vertical axis.

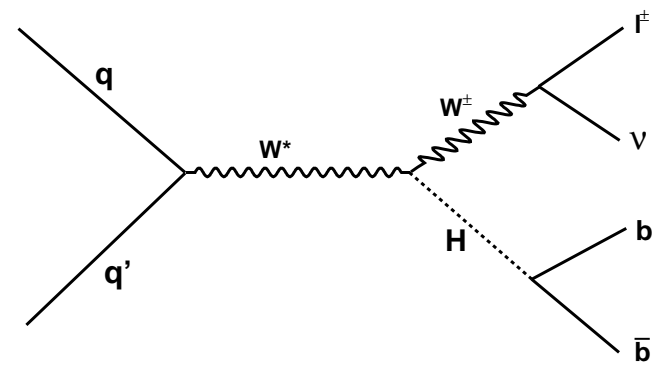

Figure 2.8: $W H$ Production and decay $(W H \rightarrow \ell \nu b \bar{b}$ ). This decay chain occurs most often for Higgs boson masses less than $135 \mathrm{GeV} / \mathrm{c}^{2}$. 


\section{CHAPTER 3}

\section{EXPERIMENTAL APPARATUS}

The Tevatron is currently the high-energy frontier. It collides protons and antiprotons $1.96 \mathrm{TeV}$ for study by two experiments, CDF and $\mathrm{D} \varnothing$. Our $W H$ search uses events recorded with the CDF detector. The Tevatron's performance and CDF's recording efficiency define the scope of our search.

\subsection{The Tevatron and the Accelerator Chain}

The Tevatron's proton and antiproton beams are prepared in a chain of accelerators. We will briefly overview the stages in the accelerator chain. Full details are available elsewhere [5]. Figure 3.1 shows the five accelerators including the Tevatron itself.

\subsubsection{Proton Acceleration}

The proton beam begins in the Cockroft-Walton pre-accelerator as hydrogen gas. The pre-accelerator ionizes the hydrogen gas $\left(\mathrm{H}^{-}\right)$and accelerates the ions to 750 $\mathrm{KeV}$ using a static electric field. It then transmits the beam to the linear accelerator (Linac).

The Linac uses radio frequency cavities (RF cavities) to accelerate the $\mathrm{H}^{-}$ions. The cavities contain electromagnetic waves that speed up the ions. The oscillating 


\section{FERMILAB'S ACCELERATOR CHAIN}

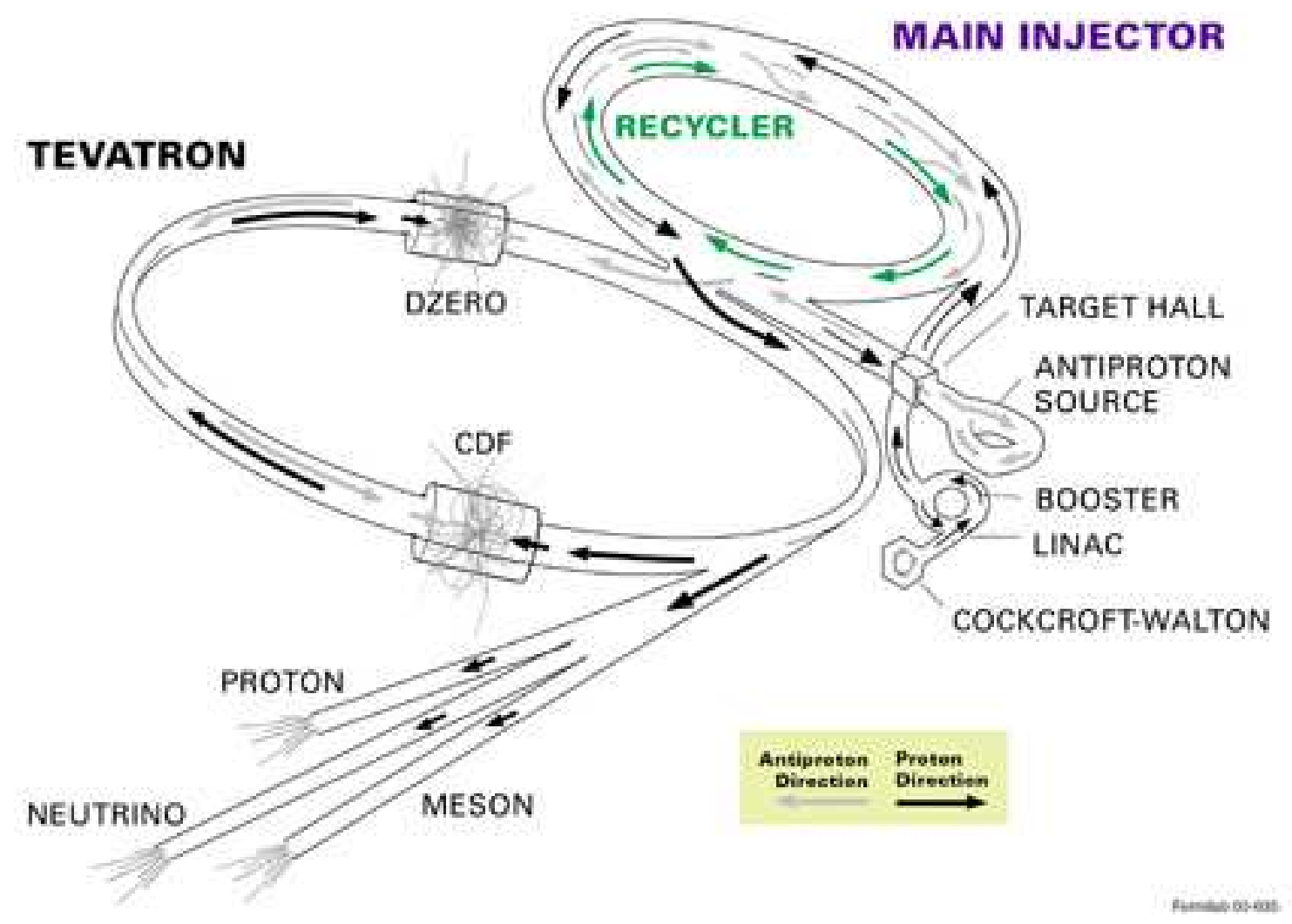

Figure 3.1: Tevatron accelerator chain and anti-proton production facilities. The Linac is $150 \mathrm{~m}$ long. The Booster has a radius of $75 \mathrm{~m}$. The Main Injector has a radius of $\sim 500 \mathrm{~m}$, and the Tevatron has a radius of $\sim 1 \mathrm{~km}$. 
waves only accelerate in-phase electrons which creates a bunched structure in the beam. RF cavities accelerate the beam at all stages in the chain except the preaccelerator. The Linac takes the ions from $750 \mathrm{KeV}$ to $400 \mathrm{MeV}$ and transfers them to the Booster. Before entering the Booster, the electrons are removed from the ions, leaving just protons.

The Booster is the first synchrotron in the accelerator chain. Synchrotrons have magnets that bend the beam in a circular path. The circular path allows the beam to pass the same accelerating RF cavities many times. The Booster accelerates the protons to $8 \mathrm{GeV}$.

The Main Injector is a synchrotron that raises the energy of the protons to either 120 or $150 \mathrm{GeV}$. Protons at $150 \mathrm{GeV}$ are transferred into the Tevatron for collisions. The $120 \mathrm{GeV}$ beam is used to seed anti-proton production.

\subsubsection{Anti-proton Production and Acceleration}

Anti-protons are created by smashing $120 \mathrm{GeV}$ protons into a nickel target. The spray of particles from the proton-nickel collision contains antiprotons at a wide range of momenta. The antiprotons are selected from the spray based on their mass and charge. The antiproton production and capture rate is low: approximately one in $10^{5}$ collisions will produce a usable antiproton. Captured protons are transferred to the Debuncher, a synchrotron designed to give the antiprotons uniform momenta. After giving each antiproton the same momenta, the Debuncher transfers the antiprotons to a larger storage ring, the Accumulator. The Accumulator builds large groups of antiprotons over many injections from the debuncher. Antiprotons are transferred from the Accumulator to the Main Injector, where they are either left 
at $8 \mathrm{GeV}$ and sent to the Recycler, or raised to $150 \mathrm{GeV}$ and set to the Tevatron. Large numbers of antiprotons are necessary to achieve the high luminosity, and the inefficiency of antiproton production is the factor limiting Tevatron luminosity. The Recycler synchrotron helps overcome the low antiproton production rate by providing additional antiproton storage capacity. The Recycler uses the same tunnel as the Main Injector.

\subsubsection{Tevatron}

The Tevatron uses the same cavities and magnets to manipulate both beams in a single beam pipe. The simplification is possible because of the proton and antiproton's opposite charge and equal mass. The Tevatron receives beams at $150 \mathrm{GeV}$ and accelerates them to $980 \mathrm{GeV}$ (center-of-mass energy $1.96 \mathrm{TeV}$ ). Super-conducting magnets and cavities made of niobium-titanium alloy provide the large fields necessary

to accelerate and bend the beams. Quadrapole magnets focus the beams into a narrow width at two interaction regions. The CDF and $\varnothing \varnothing$ experiments sit in the two interaction regions and record the collisions.

\subsubsection{Luminosity}

The intensity of the beams is described in terms of luminosity,

$$
\mathcal{L}=\frac{f N_{B} N_{p} N_{\bar{p}}}{2 \pi\left(\sigma_{p}^{2}+\sigma_{\bar{p}}^{2}\right)} F\left(\frac{d}{\beta^{*}}\right),
$$

where $f$ is the revolution frequency $(\mathrm{Hz}), N_{P}\left(N_{\bar{p}}\right)$ are number of protons (antiprotons) in each bunch, $N_{B}$ is the number of bunches, $\sigma_{p}\left(\sigma_{\bar{p}}\right)$ is RMS size of the bunches, and $F\left(d / \beta^{*}\right)$ is a form factor for the beam size depending on the bunch length $(d)$ and width in phase space $(\beta *)$. The Run II beam parameters are shown in Table 3.1. The 


\begin{tabular}{|c|c|}
\hline Parameter & Value \\
\hline Number of bunches $\left(N_{B}\right)$ & 36 \\
Protons per bunch $\left(N_{p}\right)$ & $2.7 \times 10^{11}$ \\
Antiprotons per bunch $\left(N_{\bar{p}}\right)$ & $3.0 \times 10^{10}$ \\
$\beta^{*}[\mathrm{~cm}]$ & 35 \\
Bunch length $(d)[\mathrm{m}]$ & 0.37 \\
Revolution frequency $[\mathrm{Hz}]$ & 47620 \\
Beam width $\left(\sigma_{p, \bar{p}}\right)$ & $\sim 32 \mu \mathrm{m}$ \\
$F\left(d / \beta^{*}\right)$ & $\sim 0.7$ \\
\hline
\end{tabular}

Table 3.1: Average operating parameters for the Tevatron in Run II.

maximum instantaneous luminosity for Run II is approximately. $3 \times 10^{32} \mathrm{~cm}^{-2} \mathrm{~s}^{-1}$. Figure 3.2 shows the instantaneous and integrated luminosities at CDF as a function of time. The amount of integrated luminosity defines the scope of the Higgs search because it is directly related to the number of Higgs events produced. Specifically, the number of $W H$ events $\left(N_{W H}\right)$ is

$$
N_{W H}=\sigma_{W H} \mathcal{L}_{i n t},
$$

where $\sigma_{W H}$ is the $W H$ cross section and $\mathcal{L}$ is the integrated luminosity.

\subsection{The Collider Detector at Fermilab}

The Collider Detector at Fermilab (CDF) [6] is a multipurpose detector designed to record the Tevatron collisions. It is composed of several detector sub-systems designed to measure specific event quantities. Figure 3.3 shows a cross section of the detector and labels the subsystems. 

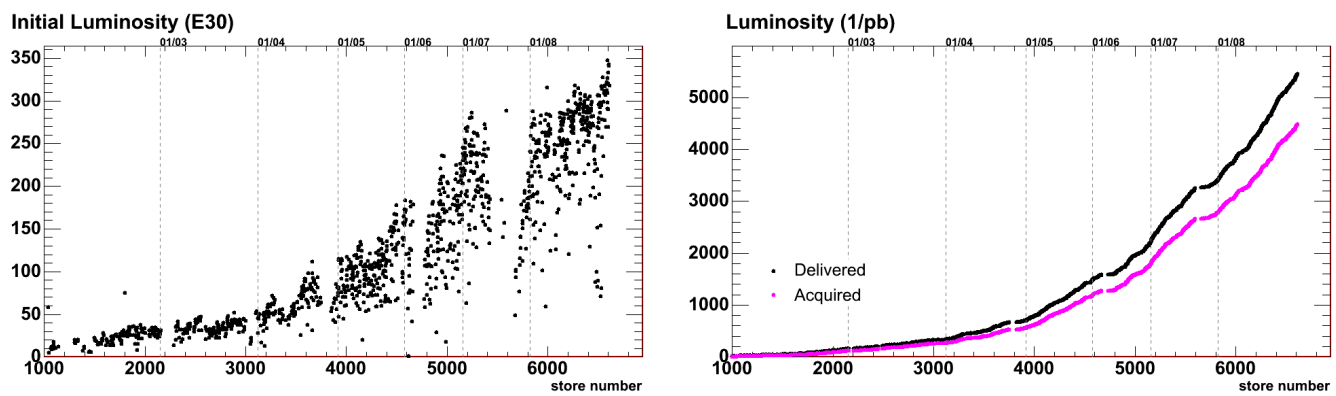

Figure 3.2: The instantaneous (left) and integrated luminosities (right) at CDF. The horizontal axis is label as store number (bottom) and fiscal year (top). The time period is from 2002 to 2008.

\subsubsection{Particle-Detector Interactions}

Figure 3.4 illustrates particle interactions with different components of the detector. Particle interaction with detector components determines our particle identification schemes. Most particles leave a signature in at least two sub-detectors. Discrimination between particles depends on correlating measurements across subsystems. The illustration shows a potential problem: most of particle signatures only differ by the measurement in a single sub-detector. Detector noise can change signatures from one particle to another and lead to an incorrect classification of events.

\subsubsection{CDF Coordinate System}

We cylindrical coordinates to describe the CDF detector. Some common quantities in this coordinate system are:

- $\theta$ is the polar angle measured relative to the proton beam direction.

- $\phi$ is the azimuthal angle in the plane perpendicular to the beam. 


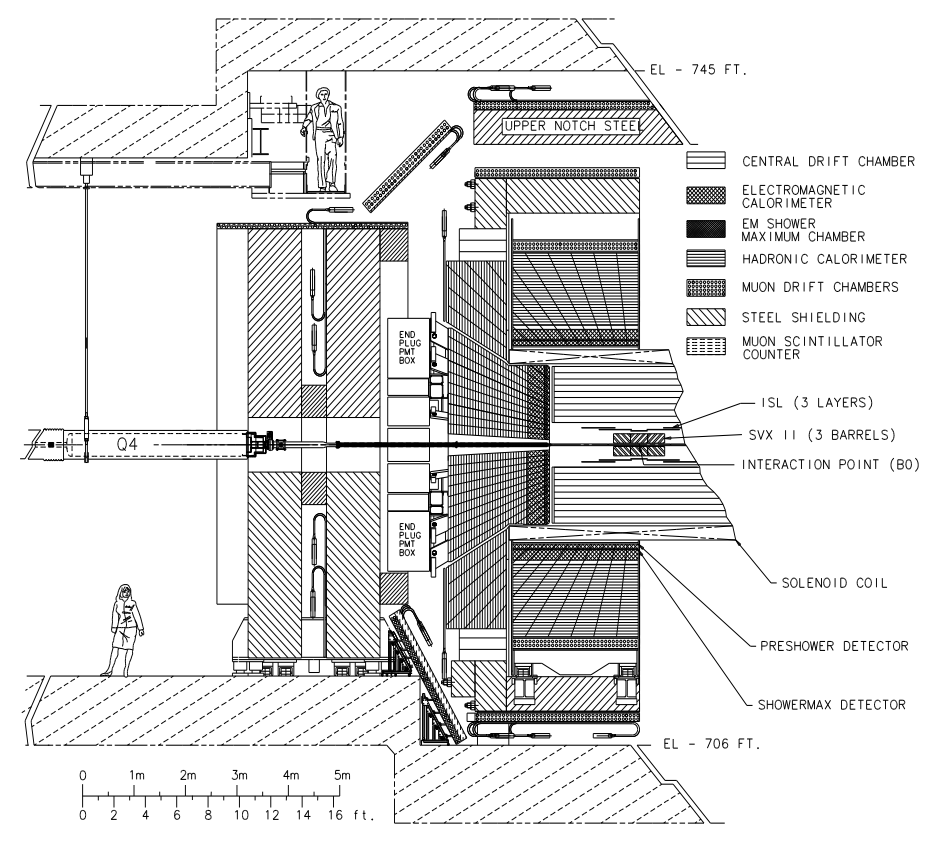

Figure 3.3: Side-view schematic of the CDF detector. The figure shows half a cross sectional view. The detector is forward-backward symmetric and azimuthally symmetric.

- $E_{T}=E \cdot \sin \theta$ is transverse energy (i.e. perpendicular to the beam)

- $P_{T}=P \cdot \sin \theta$ is transverse momentum.

- $M^{2}=E^{2}-|\vec{p}|^{2}$ is the Lorentz invariant mass of an object. The mass of a parent object that decays into two bodies can be expressed $M^{2}=\boldsymbol{P}_{\mathbf{1}} \cdot \boldsymbol{P}_{\mathbf{2}}=$ $E_{1} * E_{2}-\vec{P}_{1} \cdot \vec{P}_{2}$, where $\boldsymbol{P}_{\mathbf{1}} \cdot \boldsymbol{P}_{\mathbf{2}}$ are the four-momenta of the decay products.

- $\eta=-\ln (\tan \theta / 2)$ - is the pseudo-rapidity, and is the most common measure of angular separation from the beam line ( $\eta=0$ is perpendicular to the beam).

- $\Delta R=\sqrt{(\Delta \phi)^{2}+(\Delta \eta)^{2}}$ measures the angular separation between objects. 


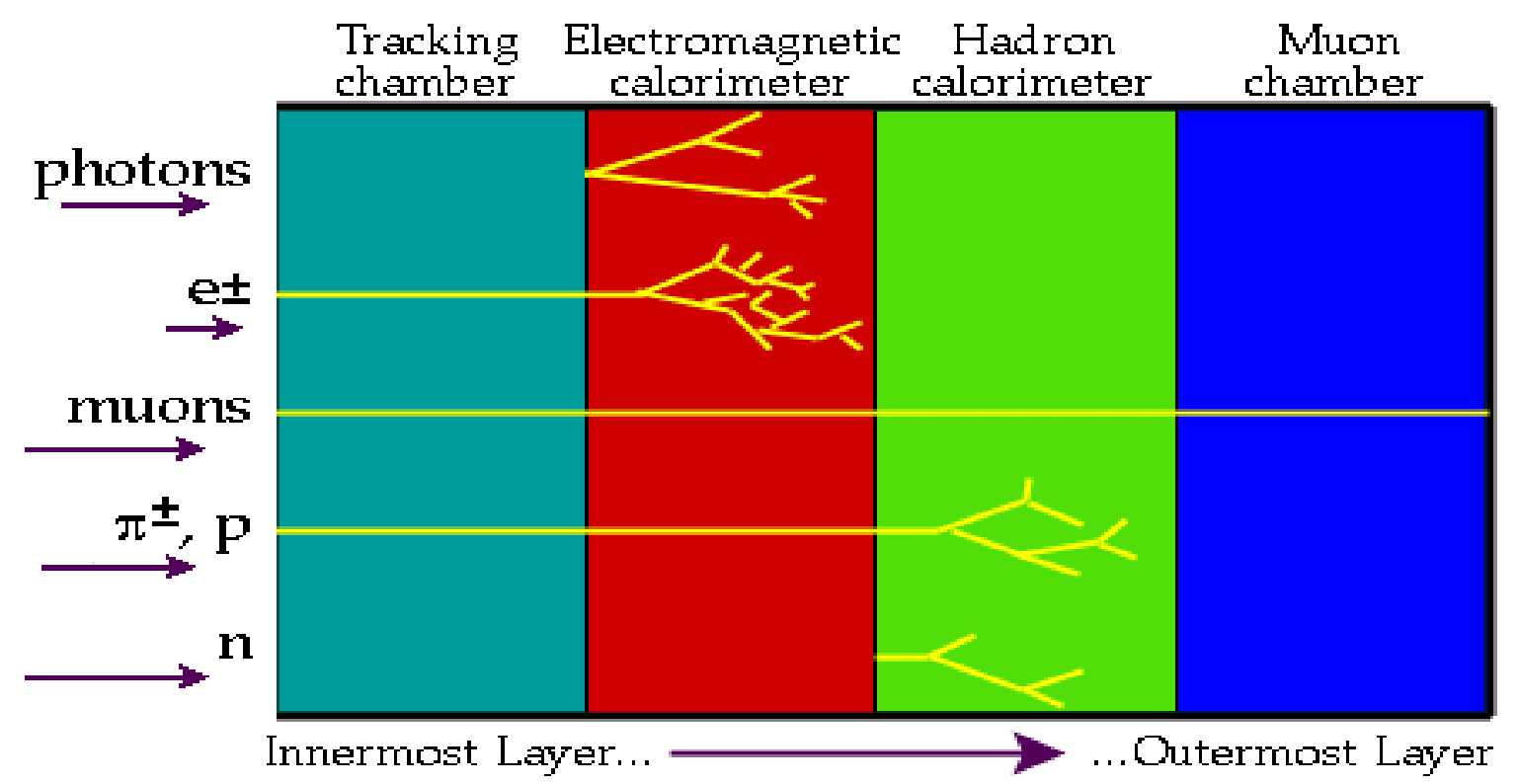

Figure 3.4: A qualitative description of particle interaction with the CDF detector.

\subsubsection{Cherenkov Luminosity Counter}

The Cherenkov Luminosity Counter (CLC) [7] lies close to the beam line in the region $3.7<|\eta|<4$.7. Particles from inelastic collisions emit Cherenkov light as they pass through the CLC's conical gas chambers. The amount of light recorded by the CLC is proportional to the number of inelastic collisions. The number of inelastic collisions, $\mu$, is related to the instantaneous luminosity, $\mathcal{L}$, according to

$$
\mathcal{L}=\frac{\mu f_{B C}}{\sigma_{\text {in }}}
$$

where $f_{B C}$ is the Tevatron bunch crossing rate and $\sigma_{i n}$ is the inelastic cross section. The CLC luminosity measurement has an error of $6 \%$. 


\subsubsection{Solenoid}

CDF's tracking volume is immersed in a 1.4 Tesla magnetic field generated by a $5 \mathrm{~m}$ long Nb-Ti super-conducting solenoid. The field is oriented parallel to the beam line. It curves the trajectory of charged particles which allows the tracking detectors to measure particle momenta and sign of charge.

\subsubsection{Silicon Tracking}

The silicon detectors measure the $\mathrm{x}, \mathrm{y}, \mathrm{z}$ position of charged particles in the region closest to the beam pipe. There are 9 layers, with the first silicon layer begins $1.35 \mathrm{~cm}$ from the outer edge of the beam pipe, and the last layer ends $28 \mathrm{~cm}$ from the beam pipe. The silicon detector consists of three separate subsystems. Layer 00 (L00) is closest to the beam pipe, followed by the five-layer Silicon Vertex Detector (SVX-II), and finally the three-layer Intermediate Silicon Layers (ISL) [8]. Figure 3.5 shows a cross section of the silicon detector and illustrates the location of the sub-detectors.

Each of the subsystems consists of several layers of silicon chips. The chips have closely spaced silicon strips that form a $p-n$ junction with the silicon substrate. Pitches vary between systems, ranging from $25 \mu \mathrm{m}$ to more than $100 \mu \mathrm{m}$. A highvoltage bias creates a large depletion region across the junction. Sensors record current produced when a charged particle ionizes an atom in the depletion region. The chips have strips on both sides. The two sides are arranged in complementary axial and stereo alignments that allow three dimensional tracking. The detector offers full tracking coverage for $|\eta|<2.0$. Impact parameter resolution is an important figure of merit for tracking systems because it is related to the system's ability to resolve 


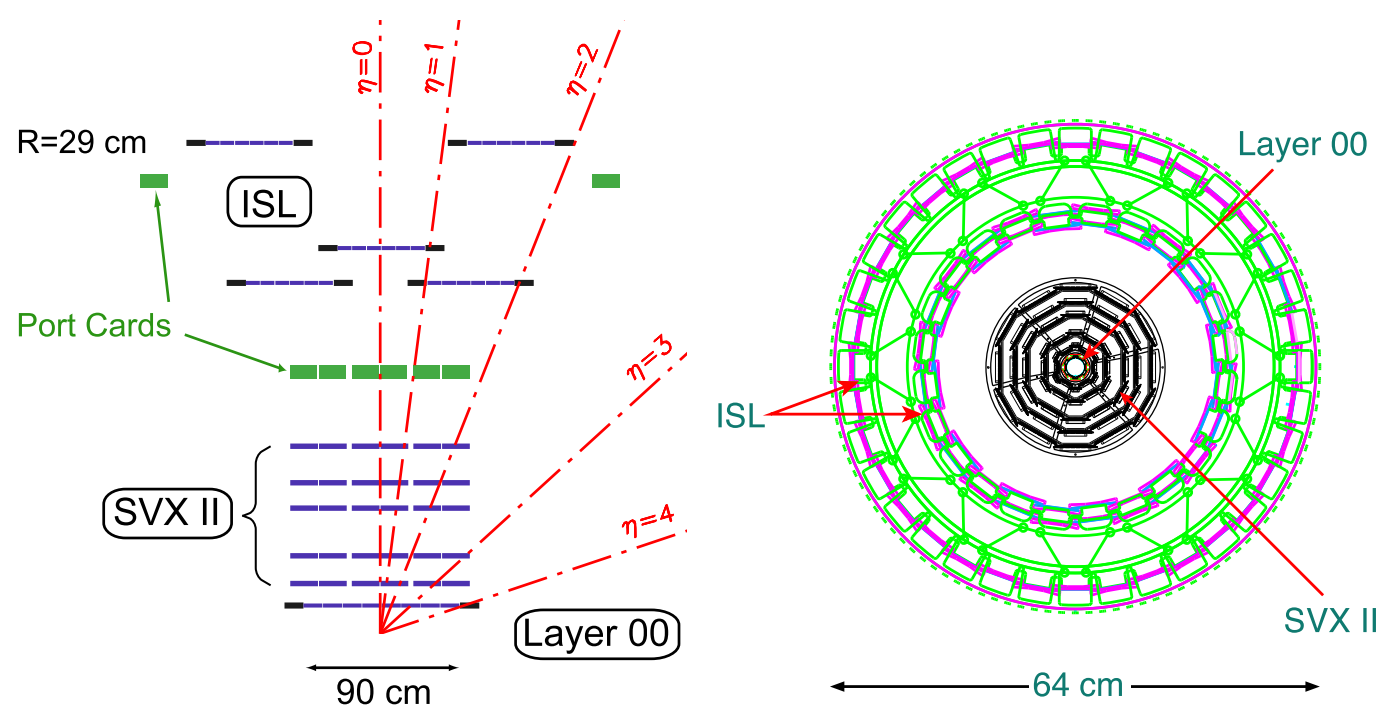

Figure 3.5: The CDF Run II silicon detector. The left figure shows the coverage provided by the silicon subsystems (full coverage is $|\eta|<2.0$ ). The right figures shows a cross section of the silicon detector.

vertices from secondary decays. The silicon system's impact parameter resolution is approximately $40 \mu \mathrm{m}$.

\subsubsection{Central Outer Tracker}

The Central Outer Tracker (COT) is a multi-wire drift chamber filled with ArgonEthane gas[9] [10]. It begins $40 \mathrm{~cm}$ from the beam pipe and extends to $132 \mathrm{~cm}$. It measures $3.1 \mathrm{~m}$ in length and provides efficient tracking coverage for $|\eta|<1.0$. The coverage of the COT and silicon detectors is illustrated in Figure 3.6. Charged particles from collisions ionize gas molecules as they pass through the COT. Wires strung along the length of the COT create an electric field that accelerates the ions toward sensor wires. The high voltage in the field wires rapidly accelerates the ions to speeds where they can ionize other gas molecules. The ions create an avalanche 


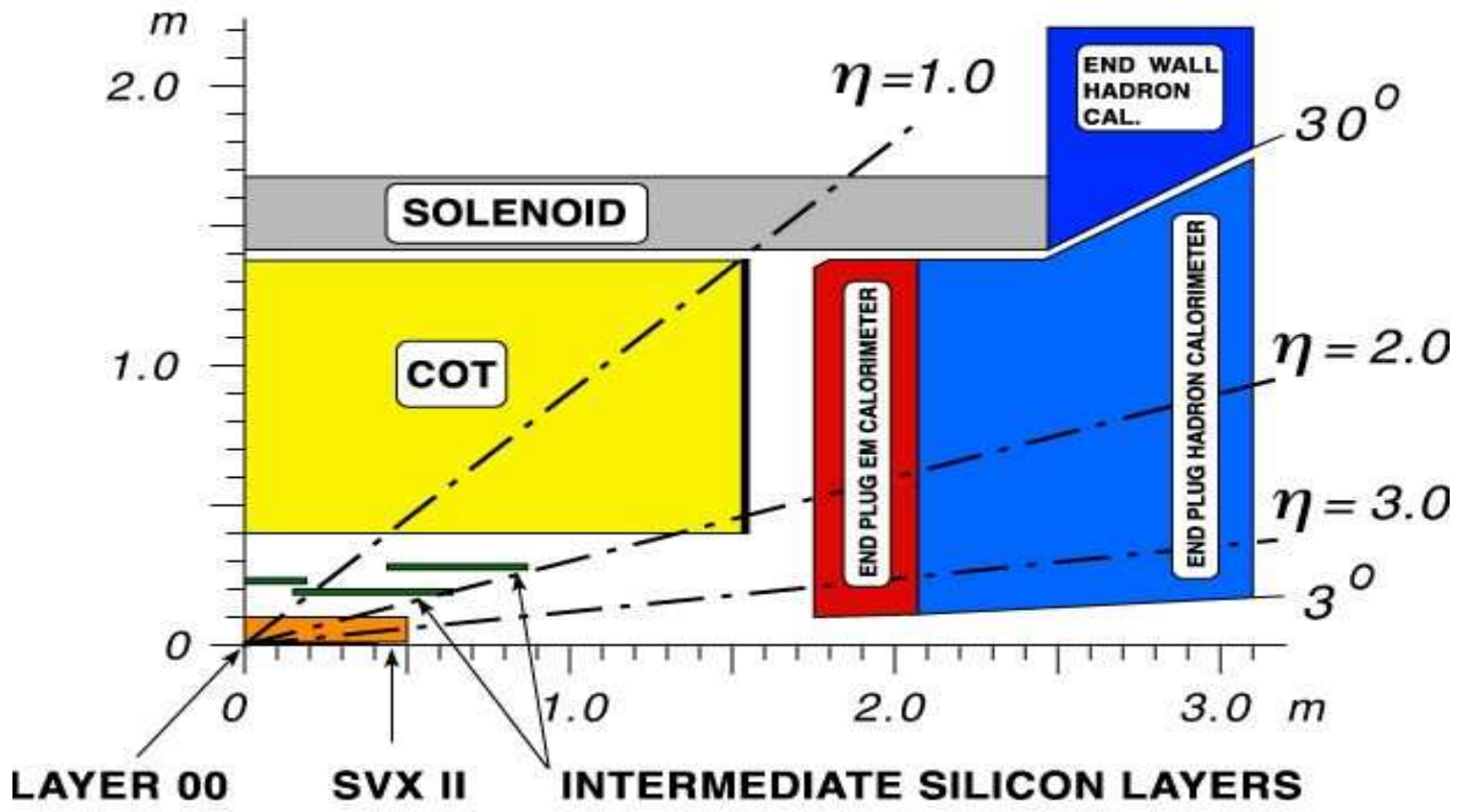

Figure 3.6: CDF tracking coverage. The figure only shows $1 / 4$ of the detector. The nominal collision point is $(0,0)$. 
of charge that falls onto the sense wires and produces a current, or hit. We reconstruct charged particle tracks using hits from several sense wires. The COT uses a complementary axial-stereo wire configuration to provide three dimensional tracking. The combined tracking resolution the COT and silicon tracking system is $\sigma_{p_{T}} / p_{T}^{2}=1.7 \times 10^{-3}(\mathrm{GeV})^{-1}$

\subsubsection{Calorimeters}

The calorimeters measure particle energies. The central calorimeter covers a region of $|\eta|<1.1$, and the plug calorimeter covers a region of $1.1<|\eta|<3.6$. Figure 3.7 shows the coverage and segmentation of the calorimetry. Each calorimeter samples particle energies using a sandwich of absorbing material and plastic scintillator. Incident particles interact with the absorbing layers and develop into a shower of many particles. The scintillator layers produce light as charged shower particles pass through. Photo-multiplier tubes read out the light from the scintillators in the sampling layers. The size of the shower inside the calorimeter indicates the energy of the incident particle. Each calorimeter has two components with different absorber materials designed to produce showers from either electromagnetic or hadronic objects. The Central Electromagnetic Calorimeter (CEM) and Plug Electromagnetic Calorimeter (PEM) use lead absorbers [11]. The CEM has an electron energy resolution of $\sigma\left(E_{T}\right) / E_{T}=13.5 \% / \sqrt{E_{T} /(\mathrm{GeV})} \oplus 2 \%$ and PEM has an electron energy resolution of $\sigma\left(E_{T}\right) / E_{T}=16.0 \% / \sqrt{E_{T} /(\mathrm{GeV})} \oplus 2 \%$. The Central Hadronic Calorimeter (CHA) and the Plug Hadronic Calorimeter (PHA) use steel absorbers [12]. The single-particle energy resolution measured with pions is $50 \% / \sqrt{E /(\mathrm{GeV})} \oplus 3 \%$ in the CHA and $80 \% / \sqrt{E /(\mathrm{GeV}} \oplus 5 \%$ in the PHA [13]. 


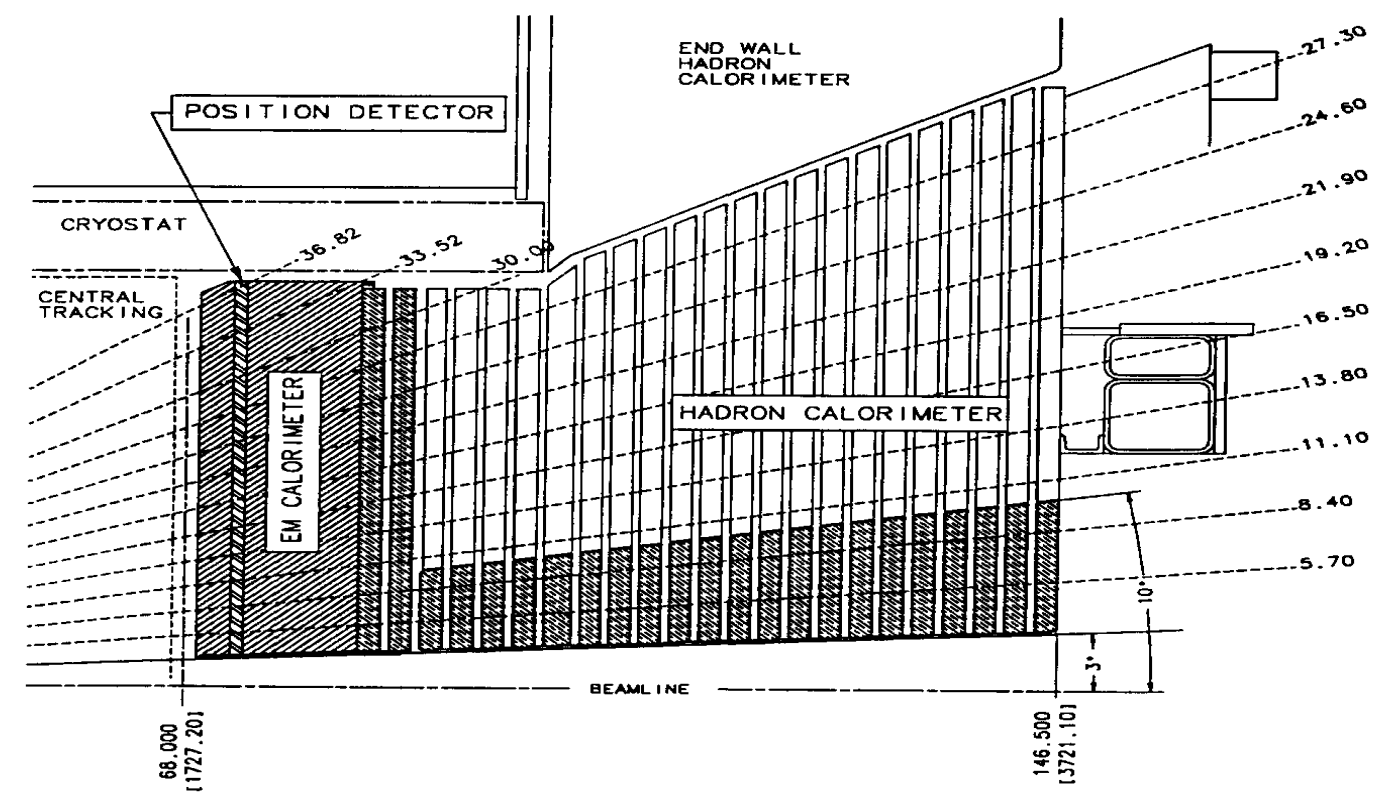

Figure 3.7: An illustration of the electromagnetic and hadronic calorimetry in CDF.

\subsubsection{Muon Detectors}

High-energy muons have a larger mass than electrons and do not shower when they pass through the detector materials. They exit the detector after depositing a minimum amount of ionization energy in the calorimeters. CDF's muon detectors are outside the other sub-detectors. They use the inner detector material as a muon filter. The muon detectors are stacks of drift chambers that identify the muon's ionization signature [14]. Hits across chambers are combined into tracks, called "stubs". Additional timing information comes from scintillators associated with the each muon detector that compensates for the chambers' long drift times. 
Each muon subsystem covers a different region of the detector. Figure 3.8 illustrates the detector coverage. The Central MUon (CMU) and the Central Muon uPgrade (CMP) cover the region of the detector with $|\eta|<0.6$. These two subdetectors are separated by $60 \mathrm{~cm}$ of steel that improves rejection of hadrons escaping from calorimeter showers. The CMU has a $2.5^{\circ} \phi$ gap between between drift cell arrays that reduces coverage to 84\%. The Central Muon Extension (CMX) covers a region of $0.6<|\eta|<1.0$. There is a fiducial $\eta$ gap between the CMUP and CMX detectors, as shown in 3.8. The Barrel Muon Upgrade (BMU) is the main detector in the Intermediate System (IMU), and it provides muon coverage in the region $1.0<|\eta|<1.5$. BMU muons do not pass through the full COT and leave only a partial track.

Our standard muon identification schemes use muons recorded by the CMU, CMP, and CMX detectors. We combine information from the central detectors $(\mathrm{CMU}+\mathrm{CMP})$ into a single muon type, CMUP. We do not use BMU muons in the current analysis.

\subsection{Data Acquisition}

The Tevatron provides collisions at a rate of $1.7 \mathrm{MHz}$. The CDF detector produces approximately $250 \mathrm{kB}$ of data for each event, or nearly half a terabyte every second. It is not practical to store all of the collision data. The CDF trigger reduces the data rate by identifying the most interesting events. The trigger reduces the rate from 1.7 $\mathrm{MHz}$ to approximately $80 \mathrm{~Hz}$ in three stages. Figure 3.9 shows the flow of data through the stages of the trigger. 


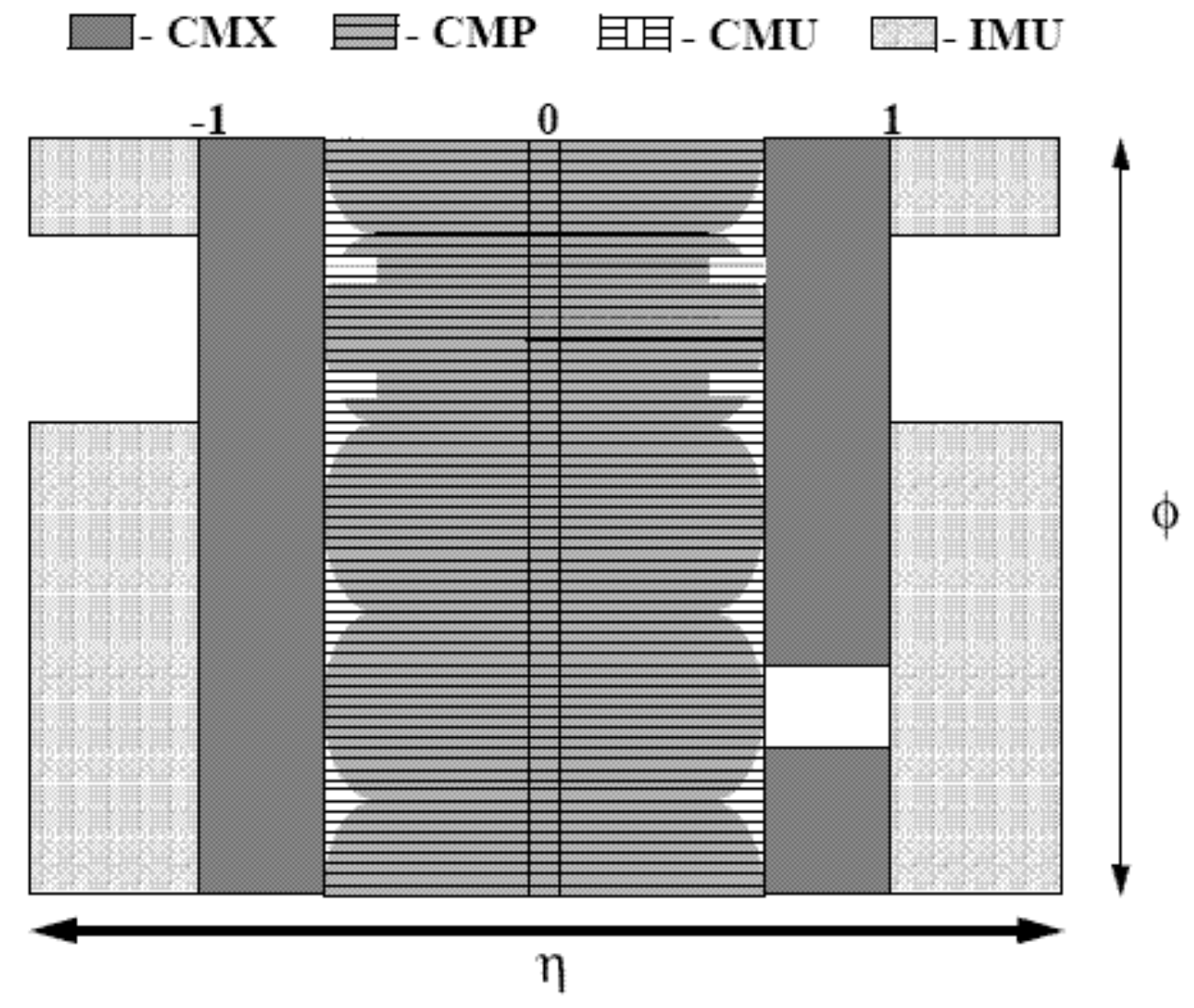

Figure 3.8: CDF muon coverage for the CMU, CMP, and BMU (referred to as IMU) detectors. The shows angular coverage in the $\phi-\eta$ plane. There are gaps between the cell arrays in the CMU and between the CMP and CMX. 


\section{Dataflow of CDF "Deadtimeless" Trigger and DAQ}

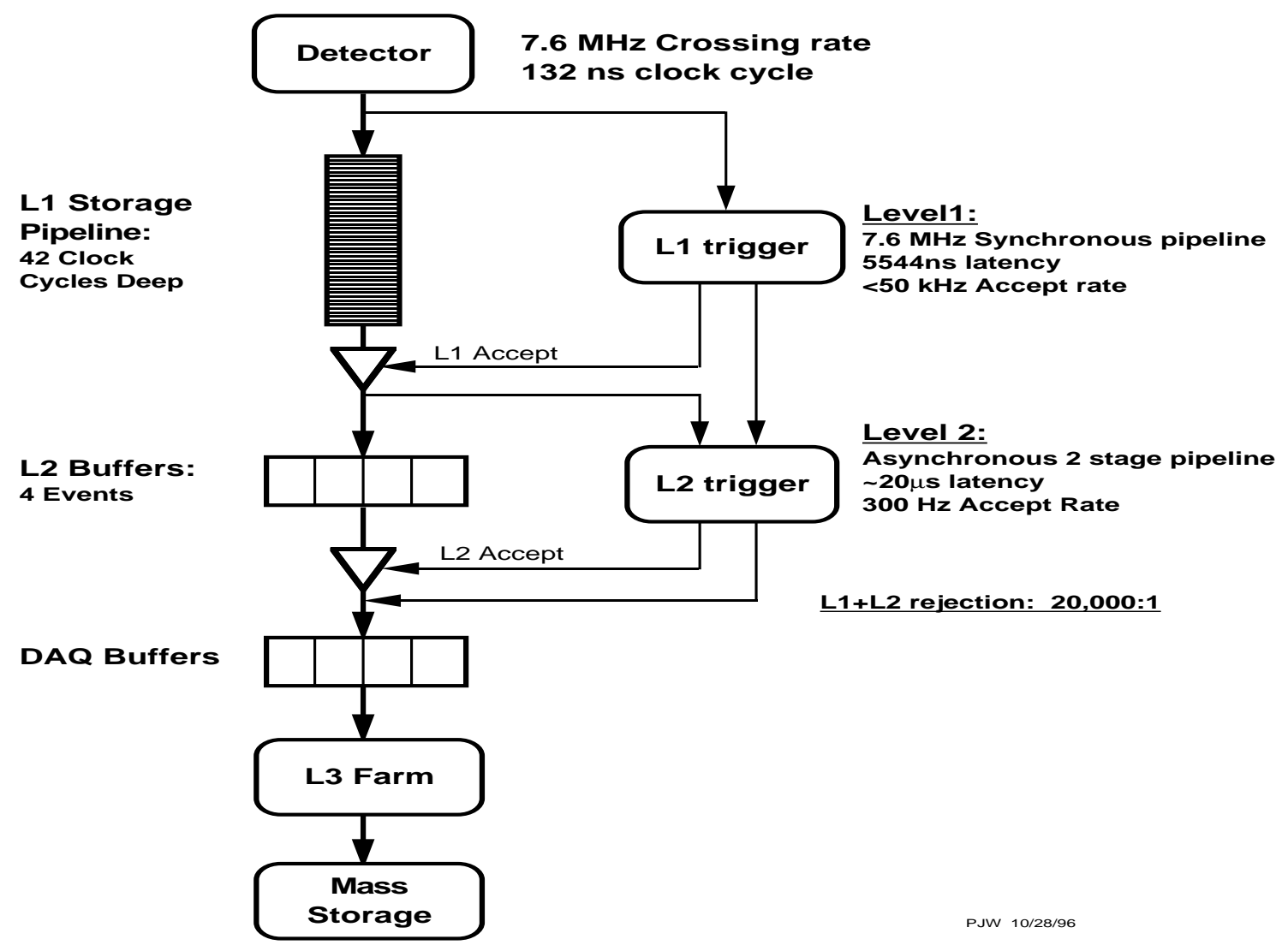

Figure 3.9: Graphical representation of the flow of CDF data through the trigger and data acquisition system. 
The Level One (L1) trigger uses custom electronics to reduce the $1.7 \mathrm{MHz}$ beam crossing rate to approximately $30 \mathrm{kHz}$. L1 uses a pipeline and buffer system that allows $2 \mu s$ to analyze each event. The L1 trigger searches for interesting physics objects, such as high- $p_{T}$ electrons, muons, jets, and missing $E_{T}$.

The eXtremely Fast Tracker (XFT) is a subsystem of the L1 trigger that identifies high- $p_{T}$ tracks in the COT. The XFT pipeline begins with a digitized readout of hits from the COT. The hits within a COT super-layer are combined into segments. Segments are combined into tracks that traverse the entire COT. Track information from the XFT is combined with information from other L1 subsystems to build physics objects, such as high- $p_{T}$ electrons and muons.

The Level Two (L2) Trigger refines the analysis of events that pass the L1 Trigger. It uses custom hardware like L1. L2 improves on Level one by incorporating silicon tracking information, improving tracking algorithms, and improving calorimeter clustering algorithms. L2 uses $20 \mu s$ of computation time to reduce the rate from $30 \mathrm{kHz}$ to $500 \mathrm{~Hz}$.

The Level Three (L3) Trigger uses software algorithms running on a farm of over $500 \mathrm{PC}$ 's. It reduces the data rate from $500 \mathrm{~Hz}$ to $80 \mathrm{~Hz}$ in 1 second of computation time. The events passed by L3 are stored for offline analysis. 


\section{CHAPTER 4}

\section{OBJECT RECONSTRUCTION}

The observable $W H$ final state consists of a high- $p_{T}$ electron or muon, missing transverse energy, and two b-quark jets. This chapter discusses the reconstruction of objects in the $W H$ final state.

\subsection{Electron Identification}

Electron candidates are identified in the central (CEM) and plug (PEM) electromagnetic calorimeters as isolated, mostly electromagnetic clusters which match a track in the pseudo-rapidity range $|\eta|<1.1$ and $1.1<|\eta|<2.0$, respectively. Clusters are made of small, contiguous groups of calorimeter towers with energy deposits. We require the cluster shape to be consistent with electron showers that were measured in test beam data. The electron transverse energy is reconstructed from the electromagnetic cluster with a precision of $\sigma\left(E_{T}\right) / E_{T}=13.5 \% / \sqrt{E_{T} /(\mathrm{GeV})} \oplus 2 \%$ for central electrons and $\sigma\left(E_{T}\right) / E_{T}=16.0 \% / \sqrt{E_{T} /(\mathrm{GeV})} \oplus 2 \%$ for plug electrons. [15].

Table 4.1 contains the electron identification cuts. The cuts are identical to those used in [16] and [17]. 


\begin{tabular}{|c|c|c|}
\hline Selection & Central $(\mathrm{CEM})$ & Plug (Phoenix) \\
\hline$E_{T}$ & $\geq 20 \mathrm{GeV}$ & $\geq 20 \mathrm{GeV}$ \\
HAD $/ \mathrm{EM}$ & $\leq(0.055+(0.00045 \times E))$ & $\leq 0.05$ \\
Isolation & $\leq 0.1$ & $\leq 0.1$ \\
Track $Z_{0}$ & $\leq 60 \mathrm{~cm}$ & $\leq 60 \mathrm{~cm}$ \\
Track $P_{T}$ & $\geq 10 \mathrm{GeV} / \mathrm{c}$ & \\
COT Axial Segments & $\geq 3$ & \\
COT Stereo Segments & $\geq 2$ & $\geq 3$ \\
Silicon Hits & $\leq 0.2$ & \\
$L_{s h r}$ & $\leq 10.0$ & $\leq 10.0$ \\
E/P & $\leq 2.0$ for $P_{T} \leq 50 \mathrm{GeV} / \mathrm{c}$ & \\
$\chi^{2}$ & $\leq 3.0 \leq Q \times \leq 1.5$ & \\
$Q \times \Delta x$ CES & $\leq 3.0 \mathrm{~cm}$ & $\leq 3.0 \mathrm{~cm}$ \\
$\Delta z$ CES & & TRUE \\
$\Delta R$ PES & & \\
Phoenix Match & &
\end{tabular}

Table 4.1: Requirements for identifying electron candidates.

\subsection{Muon Identification}

We identify muons candidates as high- $p_{T}$ tracks with minimum calorimeter ionization, isolated from other calorimeter activity, and associated with muon chamber hits. We classify muons by their recording subdetector. We require muons in the region $|\eta| \leq 0.6$ to have hits in both the CMU and CMP detectors that point back to the COT track. Muon candidates in the region $0.6 \leq|\eta| \leq 1.0$ must have CMX hits consistent with their COT track.

Table 4.2 contains the muon identification cuts.

\subsection{Isolated Track Identification}

Isolated tracks (isotrks) are generic non-triggered leptons separated from other activity in the event. The top lepton+track cross section measurement [18] originally 


\begin{tabular}{|c|c|}
\hline Selection & Cut \\
\hline$P_{T}$ & $>20 \mathrm{GeV} / \mathrm{c}$ \\
$E_{H A D}$ & $<6+\max (0,(p-100) \cdot 0.028) \mathrm{GeV}$ \\
$E_{E M}$ & $<2+\max (0,(p-100) \cdot 0.0115) \mathrm{GeV}$ \\
$E_{T}^{I s o} / P_{T}$ & $<0.1$ \\
Track $Z_{0}$ & $<60 \mathrm{~cm}$ \\
COT Axial Segments & $\geq 3$ \\
COT Stereo Segments & $\geq 2$ \\
Impact Parameter $d_{0}$ & $<0.2 \mathrm{~cm}(0.02 \mathrm{w} /$ silicon hits $)$ \\
$\chi^{2}$ & $<2.3$ \\
\hline CMU $\Delta x$ & $<3 \mathrm{~cm}$ \\
CMP $\Delta x$ & $<5 \mathrm{~cm}$ \\
CMX $\Delta x$ & $<6 \mathrm{~cm}$ \\
CMX $\rho_{C O T}$ & $>140 \mathrm{~cm}$ \\
\hline
\end{tabular}

Table 4.2: Muon selection criteria.

developed the isolated track selection criteria. The top cross section analysis used isolated track criteria to identify a second lepton candidate in dilepton top events with one triggered lepton. We use isolated tracks to identify the only lepton in the events recorded on $\not_{T}+2$-jet trigger.

Isolated track candidates are similar to the tracks associated with electron and muon candidates. Table 4.3 outlines the specific isolated track event selection criteria. We use track isolation to quantify the amount of track activity near the isolated track candidate. Track isolation frees candidates from calorimeter fiduciality requirements by using exclusively track information. It is defined as:

$$
\text { TrkIsol }=\frac{p_{T}(\text { candiate })}{p_{T}(\text { candiate })+\sum p_{T}(\text { trk })},
$$

where $\sum p_{T}(t r k)$ is the sum of the $p_{T}$ of tracks that meet the requirements in Table 4.3. Using this definition, a track with no surrounding activity has a isolation of 1.0. We require track isolation $>0.9$, or $90 \%$ of the local track $p_{T}$. 


\begin{tabular}{|l|c|}
\hline Variable & Cut \\
\hline$p_{T}$ & $>20 \mathrm{GeV}$ \\
$\eta<1.2\left|z_{0}\right|$ & $<60 \mathrm{~cm}$ \\
$\left|d_{0}\right| \operatorname{corr}$ & $<0.2$ \\
$\left|d_{0}\right| \operatorname{corr}(\mathrm{w} / \mathrm{SI})$ & $<0.02$ \\
track isolation & $>0.9$ \\
Axial COT hits & $\geq 24$ \\
Stereo Hits & $\geq 20$ \\
$\chi^{2}$ probability & $>10^{-8}$ \\
Num Si Hits (only if num expected hits $\geq 3)$ & $\geq 3$ \\
\hline
\end{tabular}

Table 4.3: Isolated track identification requirements.

\begin{tabular}{|l|c|}
\hline Variable & Cut \\
\hline$p_{T}>$ & $0.5 \mathrm{GeV}$ \\
$\Delta R$ (track, candidate $)$ & $<0.4$ \\
$\Delta Z$ (track, candidate $)$ & $<5 \mathrm{~cm}$ \\
Number of COT axial hits & $>20$ \\
Number of COT stereo hits & $>10$ \\
\hline
\end{tabular}

Table 4.4: Requirements for tracks included in Isolation Calculation 

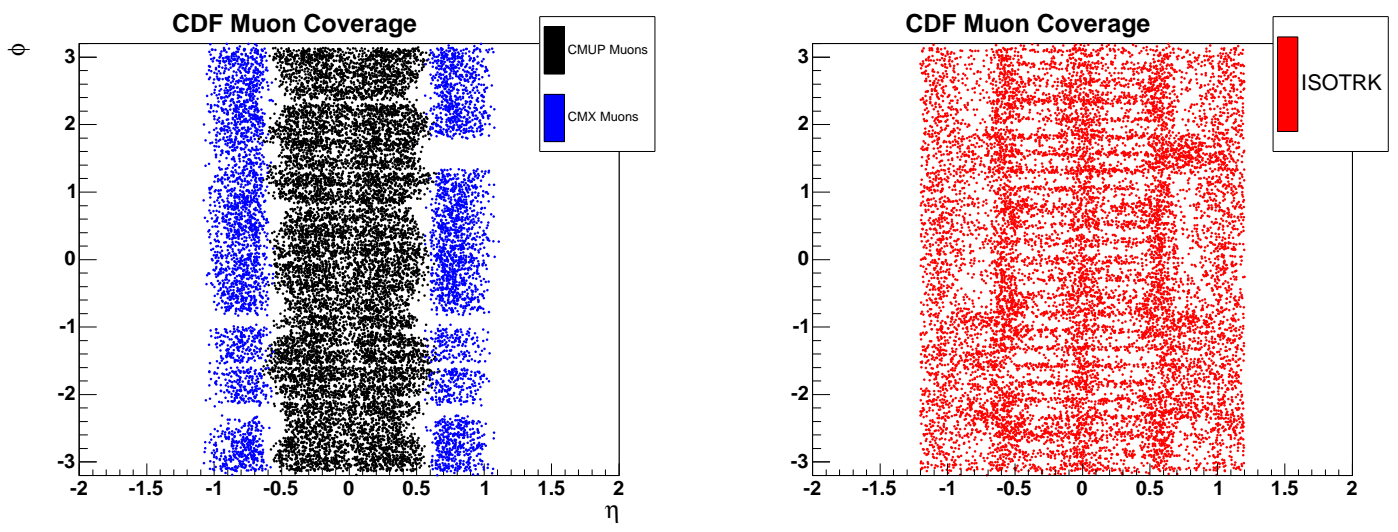

Figure 4.1: (Left) Angular distribution of muon triggered events. Note the gaps in between the CMUP and CMX acceptance. (Right) Isolated track events fill in the gaps in the muon trigger coverage. The analysis uses both lepton triggered and isolated track events.

We use vetos to ensure that isolated tracks events are from $W$ decays and that they do not overlap other lepton identifications. If any tight leptons (CEM, CMUP, CMX, PHX) are found in the event, it cannot pass isolated track selection. We veto events with two or more isolated tracks or a single isolated track that falls inside the cone of a jet $(\Delta R<0.4)$, as these events are unlikely to have come from $W \rightarrow \mu \nu$ decay.

Isolated track events compliment muon triggered events by filling in the gaps between the muon chambers and muon subsystems. Figure 4.1 shows how isolated track events increase overall muon coverage by allowing muon reconstruction in the detector gaps. Isolated track events increase acceptance by $25 \%$ relative to the tight lepton acceptance. 


\subsubsection{Isolated Track Scale factor}

A scale factor is a ratio of reconstruction efficiencies measured in data and Monte Carlo. The purpose of a scale factor is to correct the Monte Carlo reconstruction efficiency to match the data reconstruction efficiency. We measure isolated track reconstruction scale factors using a $Z \rightarrow \mu \mu$ events. To measure the scale factor we identify one high-quality $Z$ decay daughter and uses the other decay leg to probe the reconstruction efficiency in data and Monte Carlo. CMUP scale factor measurements employ an analogous method [19]. We select events with one tight CMUP or CMX muon as a tag leg and a high $p_{T}$ track as a probe leg. We further refine our sample by applying the following pre-selection cuts:

- $81<m_{l l}<101 \mathrm{GeV} / \mathrm{c}^{2}$

- $\left|\Delta z_{l l}\right|<4 \mathrm{~cm}$

- legs have opposite charge

- the tag leg fired the muon trigger (data only)

- the event is not a cosmic (passes cosmic veto)

- the probe leg satisfies $p_{T}>20$

- the probe leg has a muon stub attached

We measure the efficiency for data and Monte Carlo events passing the pre-selection cuts. The scale factor is the ratio of data to Monte Carlo efficiencies. We measure an average scale factor of 0.965 in $Z+0$ jet events. 
We estimate the uncertainty on the scale factor using several methods. We look at the dependence of the scale factor on kinematic variables. Figures 4.2 to 4.4 show the isolated track scale factor as function of $\phi, \eta$, and $p_{T}$. We estimate a $1 \%$ scale factor uncertainty from the variations in the Figures. Prior isolated track studies performed for the top cross section measurement found that the scale factor had a strong dependence on the event occupancy. The top cross section studies found that scale factors measured in $Z+2$ jet events were $4 \%$ different than scale factors measured in $Z+0$ jet events. In our study, low statistics prevented a reliable direct measurement of the scale factor in $Z+2$ jet events. We increase the uncertainty on our scale factor estimate from $1 \%$ to $6 \%$ to accommodate potential variation across jet multiplicities. Another potential source of uncertainty arises from the isolated track sample composition. WH Monte Carlo studies show that $85 \%$ of isolated tracks reconstruct $W \rightarrow \mu$ decays, but $15 \%$ of isolated tracks reconstruct $W \rightarrow e, \tau$ decays . The breakdown of the $15 \%$ total $W \rightarrow e, \tau$ is $8 \% W \rightarrow \tau$, and $7 \% W \rightarrow e$. The scale factor could vary for different lepton types, but it is difficult to accurately estimate the scale factors in the different subsamples. We assign a $25 \%$ uncertainty to the 15 $\%$ of the sample that comes from electrons and $\tau$ 's to accommodate any potential variation in the scale factor. The large uncertainty covers twice the observed variation between triggered electron and triggered muon scale factors. The total uncertainty on the isolated track scale factor acceptance is $8.85 \%$.

The small number of signal events drives the sensitivity of the $W H$ search. Including isolated track events has a large impact on the analysis sensitivity, but the isolated track scale factor uncertainty has a small impact. The scale factor uncertainty may become more significant as luminosity increases and the Tevatron transitions from 
limit to measurement. Future isolated track studies will have the opportunity to reduce the uncertainty.
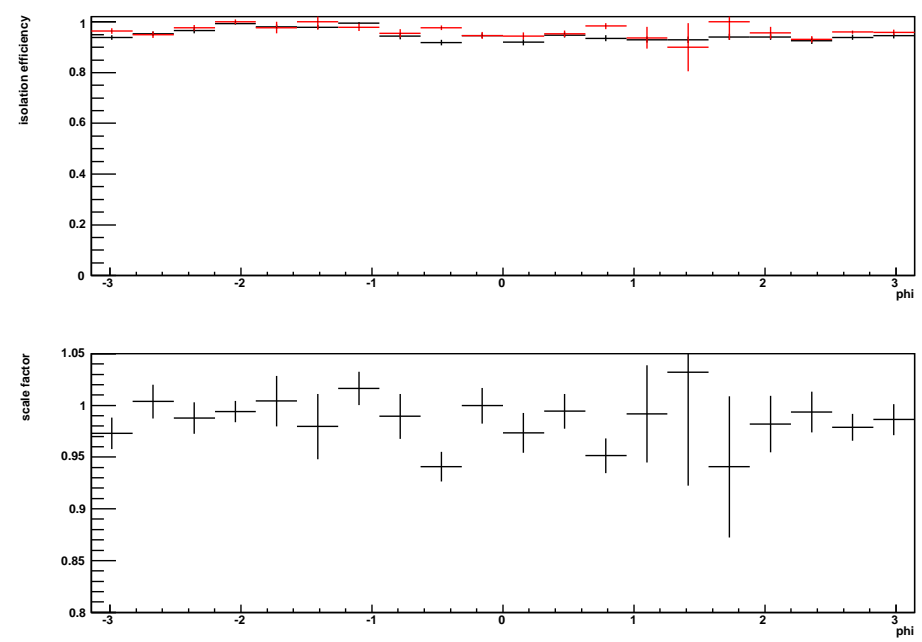

Figure 4.2: The top plot shows isolated track reconstruction efficiency in $Z$ events in data (black) and Monte Carlo (red) plotted as a function of lepton $\phi$. The isolated track reconstruction scale factor is the ratio of the data efficiency to the Monte Carlo efficiency, which is shown in the bottom plot.

\subsection{Jet Identification}

Jets are groups of electromagnetic (EM) and hadronic calorimeter clusters (HAD) which fall within a cone of radius $\Delta R \leq 0.4$ around a high- $E_{T}$ seed cluster [20]. We correct jet energies for calorimeter non-linearity, losses in the gaps between towers and multiple primary interactions. The corrections are generally called "jet corrections". The jet energy resolution is approximately $\sigma\left(E_{T}\right)=\left[0.1 E_{T} /(\mathrm{GeV})+1.0\right] \mathrm{GeV}[21]$. We classify events according to the their number of tight jets. Tight jets have $E_{T}>20$ $\mathrm{GeV}$ and $|\eta|<2.0$. Loose jets are exclusive to tight jets and fulfill a relaxed set of 

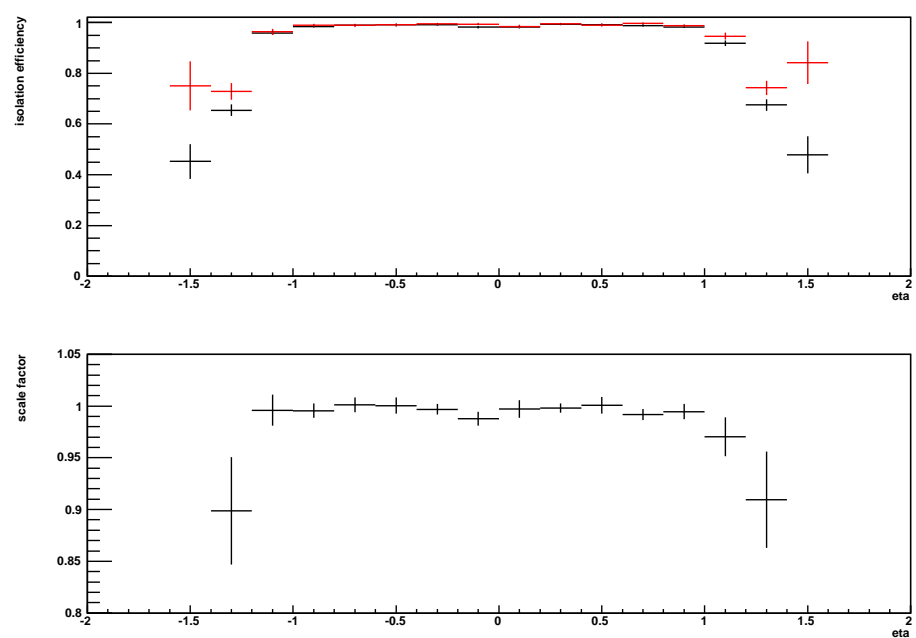

Figure 4.3: The top plot shows isolated track reconstruction efficiency in $Z$ events in data (black) and Monte Carlo (red) plotted as a function of lepton $\eta$. The isolated track reconstruction scale factor is the ratio of the data efficiency to the Monte Carlo efficiency, which is shown in the bottom plot.

criteria. They have $12 \mathrm{GeV}<E_{T}<20 \mathrm{GeV}$ and $|\eta|<2.0$, or $E_{T}>12 \mathrm{GeV}$ and $2.0<|\eta|<2.4$. We apply jet corrections to loose jets.

\subsection{Missing Transverse Energy Reconstruction}

The reconstructed missing transverse energy $\left(\mathbb{E}_{T}\right)$ is the opposite of the vector sum of all calorimeter tower energy depositions projected on the transverse plane using the primary vertex in the event as the $z$-vertex of the neutrino. It measures of the sum of the transverse momenta of undetected particles. The high- $p_{T}$ neutrino from the $W$ decay dominates the $\mathbb{E}_{T}$ magnitude in $W H$ events. The trigger hardware calculates the $\mathbb{E}_{T}$ assuming the primary vertex of the event is at the center of the CDF detector and using only calorimeter information. Offline $E_{T}$ improves on the 

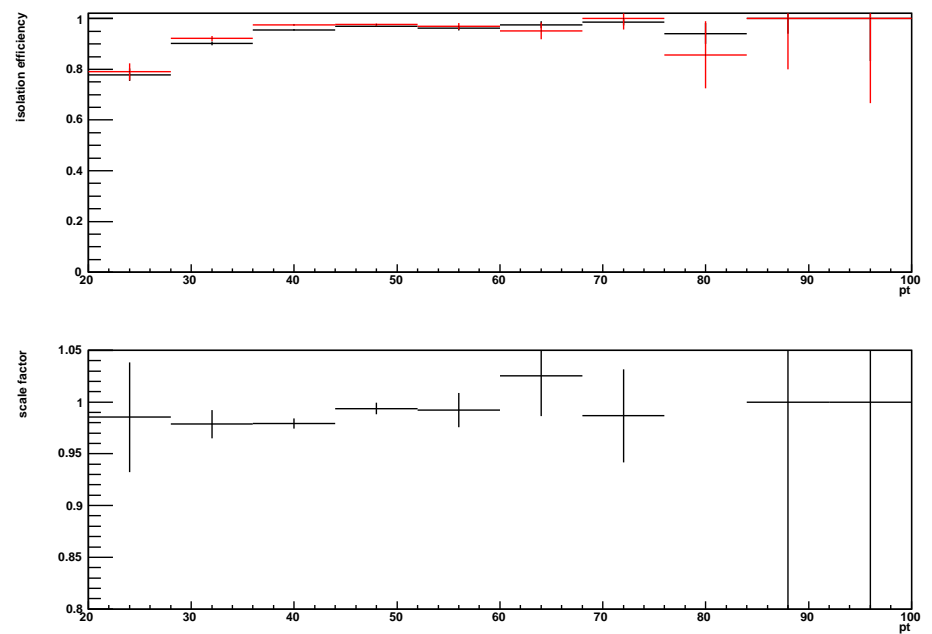

Figure 4.4: The top plot shows isolated track reconstruction efficiency in $Z$ events in data (black) and Monte Carlo (red) plotted as a function of lepton $p_{T}$. The isolated track reconstruction scale factor is the ratio of the data efficiency to the Monte Carlo efficiency, which is shown in the bottom plot.

trigger $\mathbb{E}_{T}$ in several ways. We correct offline $\mathbb{E}_{T}$ for the z-position of the primary vertex, the momentum of any minimum ionizing high- $p_{T}$ muons, and for the corrected jet energies.

\subsection{B-jet Identification Algorithms}

We use two $b$-identification algorithms introduced in Ref. [16] to optimize the selection of $b$ quark jets. Both algorithms exploit the long lifetime of $b$-hadrons to distinguish $b$ jets. $B$-hadrons travel a proper decay length of $c \tau=500 \mu m$, and typically travel a few millimeters in the lab frame before decaying. The algorithms look for tracks from particle decays significantly displaced from the $p \bar{p}$ interaction point (primary vertex). 


\subsubsection{Secondary vertex $b$-Tagging}

The Secondary Vertex (SECVTx) $b$-tagging algorithm uses tracks within a jet to reconstruct a decay vertex that is displaced from the primary vertex [22]. The algorithm uses tracks within a cone of $\Delta R=0.4$ about the jet direction. It forms vertices from tracks with large impact parameter significance $\left(\left|d_{0} / \sigma_{d_{0}}\right|\right)$ where $d_{0}$ and $\sigma_{d_{0}}$ are the impact parameter and the total uncertainty from tracking and beam position measurements. A two-pass approach tests for high-quality vertices in the first pass and allows lower-quality vertices in the second pass. The algorithm calculates the transverse distance $\left(L_{x y}\right)$ from the primary vertex and the associated uncertainty $\sigma_{L_{x y}}$, which includes the uncertainty on the primary vertex position. The sign of the transverse distance depends on the position of secondary vertex relative to the primary vertex along the direction of the jet. A negative $L_{x y}$ means the secondary vertex lies outside the jet behind the primary vertex. Negative vertex displacements are unlikely to come from $b$-hadron decays. A positive $L_{x y}$ corresponds to a vertex inside of a jet. A jet is tagged if the vertex from its tracks is significantly displaced,

$$
\begin{aligned}
& L_{x y} / \sigma_{L_{x y}} \geq 7.5 \quad \text { (positive tag) } \\
& L_{x y} / \sigma_{L_{x y}} \leq-7.5 \quad \text { (negative tag) },
\end{aligned}
$$

where the sign of the tag corresponds to the sign of $L_{x y}$.

The algorithm vetoes two-track vertices found between 1.2 and $1.5 \mathrm{~cm}$ from the center of the silicon detector (the inner radius of the beam pipe and the outer radius of the innermost silicon layer) since these vertices are likely to come from material interactions and not b-hadron decays. We reject vertices more than $2.5 \mathrm{~cm}$ from the 
center of the beam pipe since these are too many decay lengths from the primary interaction to come from $b$-hadron decays.

Not all jets tagged by the SECVTX algorithm come from $b$-quarks. Contamination can come from long-lived particles that do not contain a $b$-quark, such $D$ hadrons originating from $c$-quarks. Further contamination comes from false-tagging ("mistagging") light flavor ( $u, d, s$ or gluon) jets. We parameterize the mistag rate as a function of several variables, which we discuss in section 6.2. The large production rate of light flavor backgrounds means light-flavor mistagged events can contaminate tagged samples despite a low tag rate. The SECVTX algorithm is tuned to a low fake rate $(1-2 \%)$. Figure 4.5 shows the SECVTX mistag rate as a function of jet $E_{T}$. The efficiency for this low fake rate is approximately $40 \%$.

The efficiency for identifying a secondary vertex is different in the simulated and observed datasets. We measure an efficiency scale factor, which is defined as the ratio of the observed to the simulated efficiencies, to be $0.95 \pm 0.04$ in a sample of high- $E_{T}$ jets enriched in $b$ jets by requiring a soft lepton $\left(p_{T}>8 G e V\right)$ from semi-leptonic heavy quark decays [22]. Figure 4.6 shows the $b$-tag efficiency and as a function of jet $E_{T}$.

\subsubsection{Jet Probability $b$-tagging}

The Jet Probability algorithm analyzes track impact parameters to distinguish $b$ jets from light flavor jets. The tracks in light flavor jets will have impact parameters consistent only with the primary vertex. The tracks in $b$-jets will have large impact parameters that are consistent with a displaced vertex. 


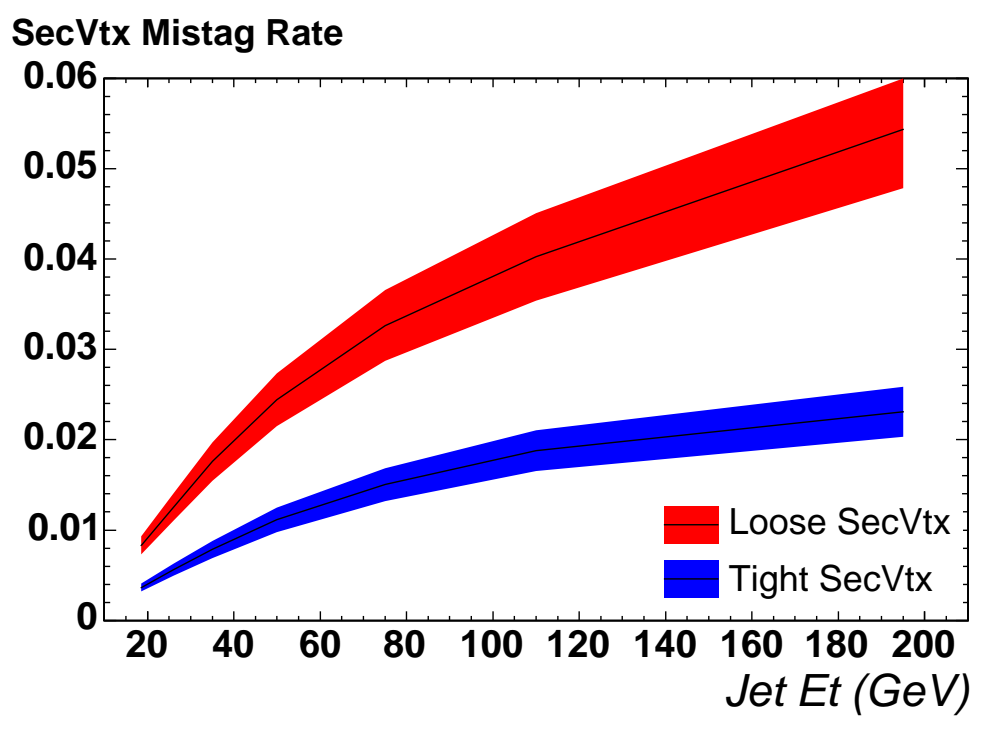

Figure 4.5: The right-hand plot shows the SECVTX mistag efficiency from inclusive jet data as function of jet $E_{T}$. Our analysis uses only tight tags, shown in blue. The mistag rate includes an asymmetry correction to the negative tag rate that accommodates material interactions. 


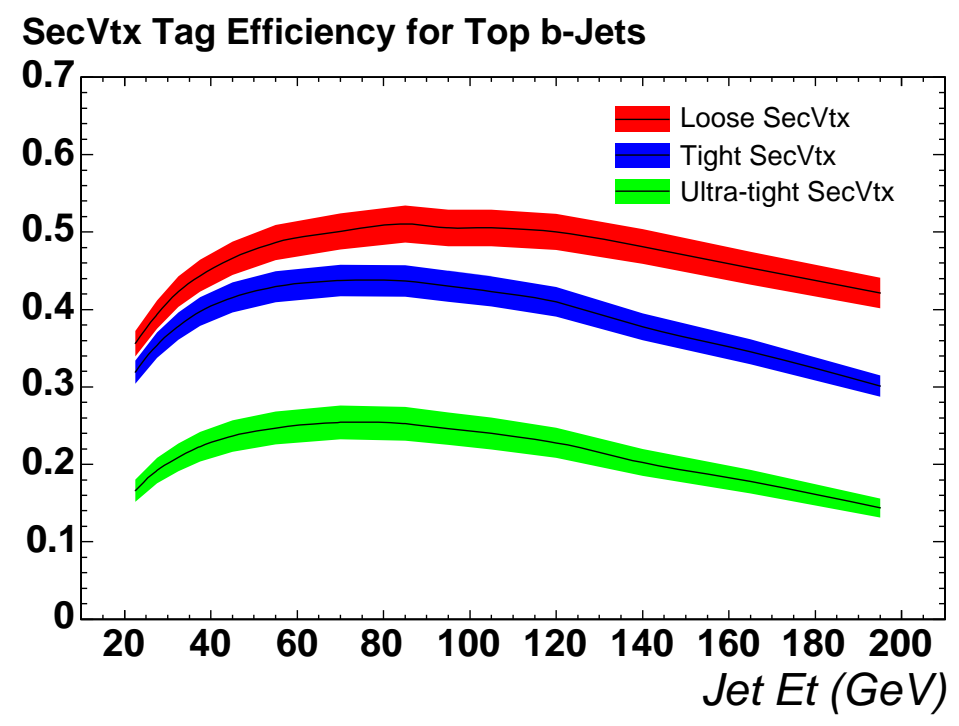

Figure 4.6: The plot shows the SECVTX tag efficiency for $b$-jets in $t \bar{t}$ Monte Carlo and plotted as a function of jet $E_{T}$. Our analysis uses only tight tags, which are shown in blue. The Monte Carlo efficiency is corrected by a constant scale factor to match the tag rates from data. 
Figure 4.7 (left) illustrates a jet consisting of two tracks and their impact parameters. The sign of impact parameters is assigned the same way as vertex displacement. Measurement resolution has an unbiased effect on the track impact parameters. Tracks orgininating at the primary vertex have a equal probability to be either sign, as shown in Figure 4.8 (left). Tracks from secondary vertices, however, will be biased toward positive impact parameters, as illustrated with the track shown in Figure 4.7 (right). Accordingly, jets with secondary vertices with have a positively biased impact parameter distribution such as the one shown in Figure 4.8 (right).
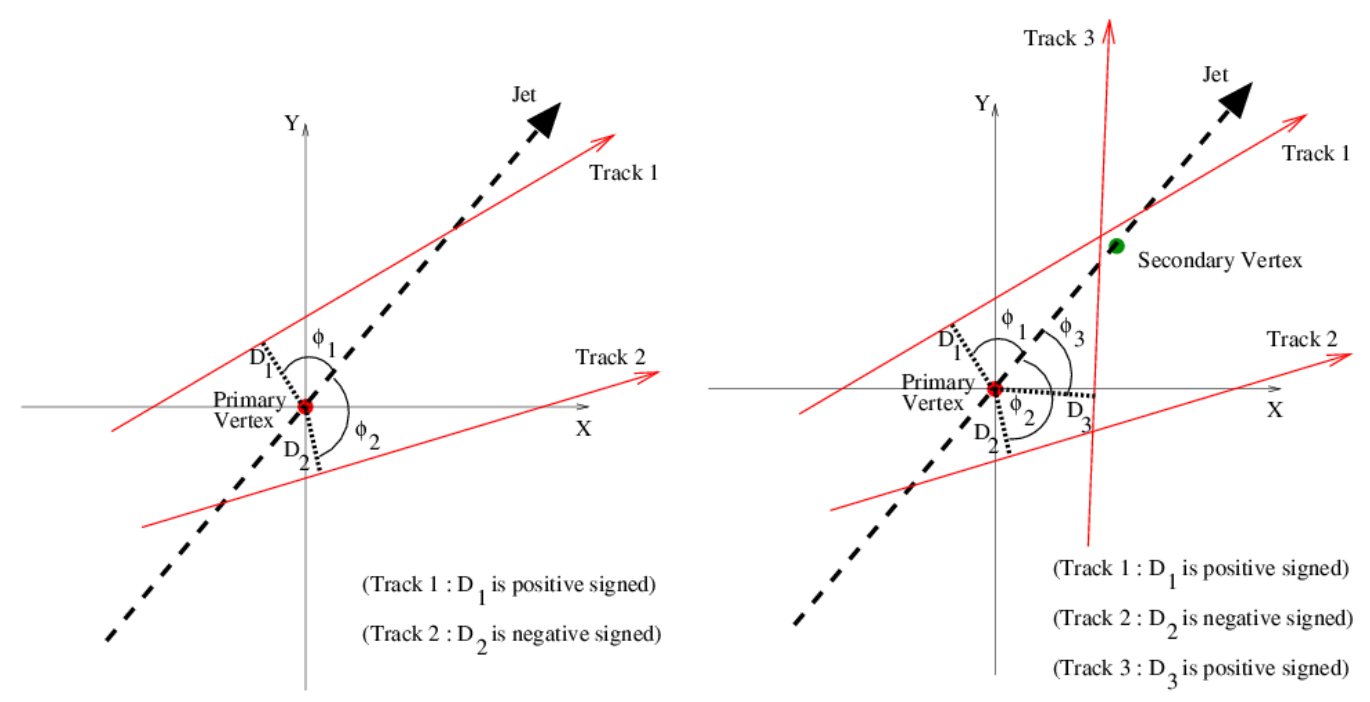

Figure 4.7: Track impact parameters shown in the plane transverse to the beam. The primary vertex is at the origin, and impact parameters are measured as displacement from the origin. The dashed black line indicates the direction of the jet. Tracks from the primary vertex (left) are equally likely to have positive $\left(\phi<90^{\circ}\right)$ or negative $\left(\phi>90^{\circ}\right)$ impact parameters. Tracks from a secondary vertex (right) are more likely to have positive . 

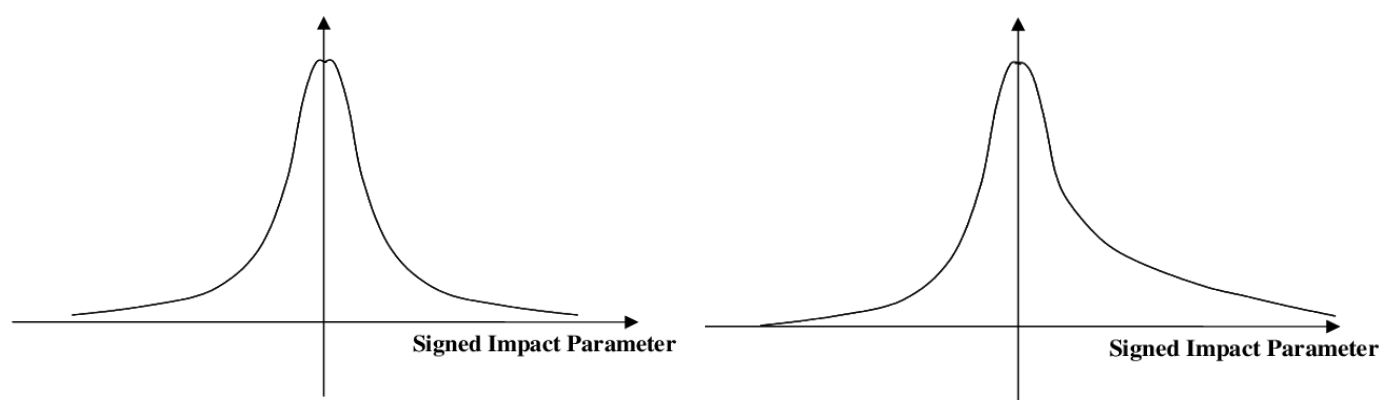

Figure 4.8: Distribution of track impact parameters from negative to positive. Tracks from a primary vertex (left) are symmetric about the origin, indicating that they are equally likely to have either positive or negative impact parameters. Tracks from a secondary vertex (right) have a distribution that is biased towards positive impact parameters.

The probability for a jet to have a secondary vertex is

$$
P_{j e t}=\Pi \sum_{k=0}^{N-1} \frac{(-\ln \Pi)^{k}}{k !},
$$

where

$$
\Pi=P_{1} P_{2} P_{3} \cdots P_{N}
$$

and $P_{1} \ldots P_{N}$ is the probability that an individual track is consistent with a secondary vertex. The individual track probabilities are expressed as a function of the impact parameter resolution,

$$
P_{\text {track }}\left(S_{d_{0}}\right) \equiv \int_{-\infty}^{-\left|S_{d_{0}}\right|} R(t) d t
$$

where $R(t)$ is the impact parameter resolution, $S_{d_{0}}$ and $t$ are signed impact parameter significance. We obtain the resolution parameterization from fitting tracks in jet data. The fit uses only negative signed impact parameter tracks, and assumes a symmetric distribution for positive signed impact parameters in light flavor jets. Tracks from the primary vertex will have a track probability that is flat between zero and one. 
Tracks from long-lived particles will have a probability that peaks at zero (i.e., they are unlikely to have come from the primary vertex). Since the Jet Probability is a product of track probabilities, it is also peaked at 0 for jets tracks from long-lived particles. We place a cut on the Jet Probability distribution at an operation point where the jet fake rate is $5 \%$ and $b$-tagging efficiency is $60 \%$. We use a scale factor to capture the difference in performance between data and Monte Carlo. We measure the scale factor to be $0.85 \pm 0.07$ in a sample of high- $E_{T}$ jets enriched in $b$-quarks by requiring a soft lepton from a semi-leptonic $b$-decay [23]. The uncertainty in the scale factor accounts for the $E_{T}$ dependence of the tagging efficiency in data and Monte Carlo.

Jet Probability tagging is susceptible to the same fake sources as SECVTX tagging. We use a separate fake parameterization for SECVTX fakes and Jet Probability fakes. 


\section{CHAPTER 5}

\section{EVENT SELECTION AND DATASET}

Our results use data collected between February 2002 and April 2008. We analyzed $2.7 \mathrm{fb}^{-1}$ of data recorded with the high- $p_{T}$ central electron and muon triggers, and the missing $E_{T}$ plus 2 jets trigger $\left(E_{T}+2\right.$ jets $)$. We treat each trigger dataset separately and combine them together for our final result. This chapter discusses the trigger details and the event cuts we apply to confirm the $W H$ decay signature.

\subsection{Trigger Efficiencies}

The high- $p_{T}$ central electron and muon triggers have a Level Three trigger threshold of $p_{T}>18 \mathrm{GeV} / \mathrm{c}$. We require our leptons have $p_{T}>20 \mathrm{GeV} / \mathrm{c}$, where the trigger has reached plateau efficiency. Table 5.1 shows the efficiency for events to pass the CEM, CMUP, and CMX triggers.

\begin{tabular}{|l|c|}
\hline Trigger & Efficiency \\
\hline CEM & $.9691 \pm .0042$ \\
CMUP & $.915 \pm .005$ \\
CMX & $.9276 \pm .0034$ \\
\hline
\end{tabular}

Table 5.1: High $p_{T}$ lepton trigger efficiencies. 
We record plug electron events on the $\mathbb{E}_{T}+$ PEM trigger. The trigger's baseline requirement is $\mathbb{E}_{T}>15 \mathrm{GeV}$ and electron $E_{T}>20 \mathrm{GeV}$. We require offline electron $E_{T}>20 \mathrm{GeV}$ and we parameterize the trigger turn-on as a function of the trigger $E_{T}$, electron $\eta$, and electron $E_{T}$. We use the same $\mathbb{E}_{T}+$ PEM trigger parameterization employed in earlier $W H$ searches [16] and single top measurements [24].

The $\not_{T}$ plus two jets trigger has been used in the $V H \rightarrow \not_{T}+b \bar{b}$ Higgs search [25] and offers a chance to reconstruct $W H$ events that did not fire the high- $p_{T}$ lepton trigger. The trigger's baseline requirements are a central jet $\left(|\eta|<0.9, E_{T}>10\right.$ $\mathrm{GeV})$, a second jet $\left(E_{T}>10\right)$, and $E_{T}{ }^{\text {raw }}>35 \mathrm{GeV}$ where $E_{T}{ }^{\text {raw }}$ assumes that the primary vertex of the event is at the center of the detector and does not correct for any muon transverse momentum. The kinematics of the jets have a strong influence on the $E_{T}$ reconstruction. We require the two leading jets to be well-separated $(\Delta R>1.0)$ and have a large $E_{T}$ (offline corrected $E_{T}>25 \mathrm{GeV}$ ) to ensure that the $\mathbb{E}_{T}$ is well measured. The cuts on the jets allow us to parameterize the $\mathbb{E}_{T}$ trigger turn-on as a function $\mathbb{E}_{T}$ corrected for the primary vertex position $\left(\mathbb{E}_{T}\right.$ vertex $)$, as shown in figure 5.1. We weight each isolated track Monte Carlo event by it's efficiency to pass the $E_{T}$ trigger.

We use CMUP-triggered events to measure the $\mathbb{E}_{T}$ trigger efficiency and uncertainty. We divide the CMUP-triggered sample into subsamples based on run number and jet kinematics, then measure the $E_{T}$ turn-on curve for each subsample. The uncertainty is the variation in weighted $W H$ acceptance between the nominal and the subsample turn-on curves. We sum the uncertainties from the sub-samples in quadrature. The total uncertainty is $3 \%$. 


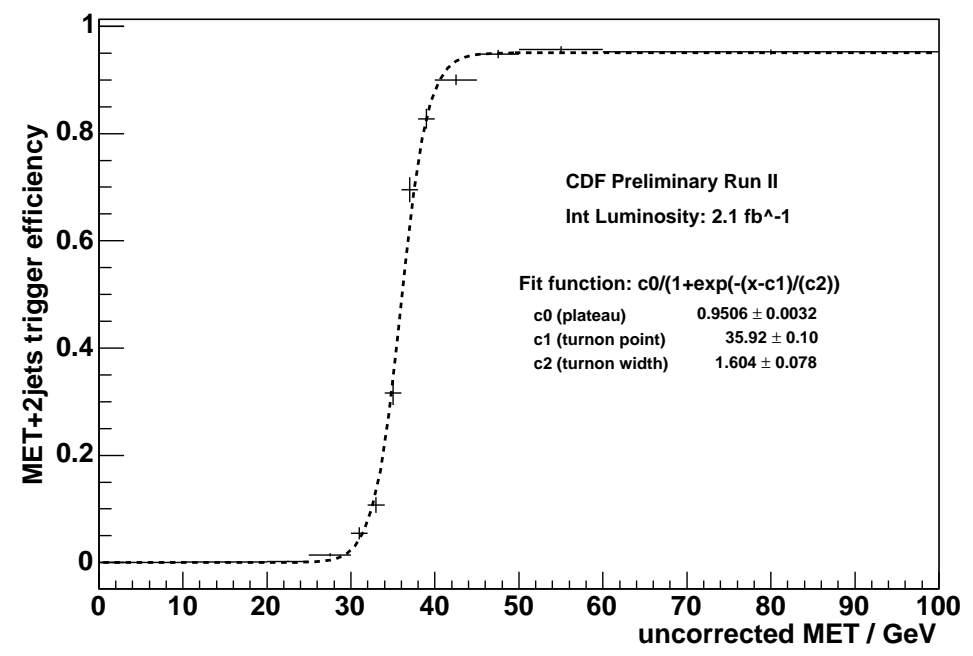

Figure 5.1: $\mathbb{E}_{T}$ plus jets trigger turn-on curve parameterized as a function of vertex $E_{T}$.

\subsection{Event Selection Requirements}

We select events from each trigger stream with a single, high- $p_{T}(>20 \mathrm{GeV} / \mathrm{c})$, isolated lepton consistent with leptonic $W$ decay. The electron and muon identification is applied to events recorded on the respective high- $p_{T}$ triggers.

After identifying the lepton in the event as a tight central lepton, plug electron or isolated track, we purify the sample with quality cuts. We fit a subset of quality tracks coming from the beam line to determine the event's primary vertex. The lepton track $z_{0}$ must be within $5 \mathrm{~cm}$ of the primary vertex to ensure the lepton and the jets come from the same hard interaction. We reduce backgrounds from $Z$ decays with unidentified leptons by vetoing events where the invariant mass of the lepton and a second track with $p_{T}>10 \mathrm{GeV} / c$ falls in the $Z$-boson mass window 
$\left(76<m_{\ell X}<106 \mathrm{GeV} / c^{2}\right)$. We confirm the $W$ decay signature by requiring at least $20 \mathrm{GeV}$ of $\not_{T}$.

$W H$ signal events have two high- $E_{T}$ jets originating from $H \rightarrow b \bar{b}$ decays. We require $W H$ candidate events to have exactly two high- $E_{T}$ jets, $E_{T}>20 \mathrm{GeV}\left(E_{T}>\right.$ 25 on the $\not_{T}+2$ Jets trigger) and $|\eta|<2.0$.

We require at least one jet to $b$-tagged by the SECVTX algorithm. To maximize our sensitivity, we break the sample of events with at least one tag into three exclusive tag categories of varying purity. Events with a second SECVTX tagged jet have the highest purity. If the event does not have a second SECVTX tag, then we look for a Jet Probability tag. The remaining events have exactly one SECVTx tag. One tag events have largest event yield but worst signal to background ratio.

QCD contamination comes from events without a true $W$-boson. The false $W$ signature comes from a jet faking a lepton and overall mismeasurement faking $\mathbb{E}_{T}$. $B$-tagging reduces QCD contamination, but extra kinematic rejection is important to increase the purity of events with only one $b$-tag. The kinematics of the QCD contamination vary with the lepton signature they mimic. We apply a separate veto to each lepton subsample. Table 5.2 details the QCD vetos for each lepton type. The vetoes reduce the QCD fraction in each sample by roughly by roughly $50 \%$.

The kinematic variables used in the vetos are correlated with mismeasurement and discriminate QCD fakes from true $W$ 's. $M E T_{\text {sig }}$ encompasses the difference between the raw and corrected $E_{T}$. It is defined as:

$$
M E T_{s i g}=\frac{E_{T}}{\sqrt{\sum_{j e t s} C_{J E S}^{2} \cos ^{2}\left(\Delta \phi_{j e t, M E T}\right)+\cos ^{2}\left(\Delta \phi_{v t x, c o r r}\right)}},
$$




\begin{tabular}{|l|c|}
\hline Quantity & Cut \\
\hline \multicolumn{2}{|c|}{ CEM } \\
\hline$M_{T}$ & $>20 \mathrm{GeV} / \mathrm{c}^{2}$ \\
$M E T_{\text {sig }}$ & $\geq-0.05 \cdot M_{T}+3.5$ \\
$M E T_{\text {sig }}$ & $\geq 2.5-3.125 \cdot \Delta \phi_{M E T, j e t 2}$ \\
\hline \multicolumn{2}{|c|}{ CMUP,CMX } \\
\hline$M_{T}^{W}$ & $>10 \mathrm{GeV}$ \\
\hline \multicolumn{2}{|c|}{ PHX } \\
\hline$E_{T}$ & $>25 \mathrm{GeV}$ \\
$M E T_{\text {sig }}$ & $>2.0$ \\
$E_{T}$ & $>45-30 \cdot\left|\Delta \phi_{M E T, \text { Jet } 1}\right|$ \\
$E_{T}$ & $>45-30 \cdot\left|\Delta \phi_{M E T, \text { Jet } 2}\right|$ \\
\hline \multicolumn{2}{|c|}{ ISOTRK } \\
\hline$M_{T}$ & $>10 \mathrm{GeV}$ \\
\hline
\end{tabular}

Table 5.2: QCD veto cuts for each lepton category. These cuts are applied to events with exactly one identified b-jet.

where $C_{J E S}$ is the jet energy correction factor; $\Delta \phi_{v t x, \text { corr }}$ is the azimuthal angle between corrected and uncorrected missing transverse energy. $M E T_{\text {sig }}$ is used the electron QCD vetos listed in Table 5.2. Transverse mass related to the invariant mass $M^{2}=E^{2}-|\vec{p}|^{2}$. Transverse mass is defined as,

$$
M_{T}(W)=\sqrt{2 p_{T}^{l e p} E_{T}-\boldsymbol{p}_{T}^{l e p} \cdot \boldsymbol{E}_{T}} .
$$

Transverse mass is used in the QCD vetos listed in Table 5.2. 


\section{CHAPTER 6}

\section{BACKGROUND MODELING}

Associated Higgs production shares the $\ell \nu b \bar{b}$ final state with a number of background processes. The dominant backgrounds are $W+$ jets production, $t \bar{t}$ production, single top production, and QCD multi-jet production (non- $W$ ). Diboson production and Z+jets production, collectively referred to as "electroweak backgrounds", contribute to the sample at smaller rates. Diboson production has a small contribution because of its small cross section and, in the case of WW, lack of $b$-jets. Z+jets production has a small contribution because our analysis cuts, such as the requirement for exactly one tight lepton, give it a small overlap with our final state. Our estimate of the background rates uses a combination of Monte Carlo techniques and extrapolations from regions where the backgrounds are well understood. The estimate is based CDF's single top searches [24], and was used in the prior $W H$ search [26]. We will briefly overview the background estimate, then discuss the details of each background in the subsections that follow.

Our background estimate begins in the $W+$ jets sample before applying tagging requirements, which we call the "pretag" sample. We use this sample where the background is well understood and the signal contribution is small, called a sideband, to estimate our $\mathrm{W}+$ jets background rates, which we then extrapolate to the signal 
region. First, we use a Monte Carlo technique to estimate the relative contribution of processes whose rates and topologies are well described at tree-level. These processes include $t \bar{t}$, single top and diboson production. We estimate their expected contribution using the theoretical cross section $(\sigma)$, Monte Carlo event detection efficiency corrected to match the efficiency in the data $(\epsilon)$, and the integrated luminosity of our dataset $\left(\mathcal{L}_{\text {int }}\right)$ :

$$
N_{\text {top }, \text { ewk }}=\sigma_{\text {top }, \text { ewk }} \cdot \epsilon \cdot \mathcal{L}_{\text {int }}
$$

We subtract the contribution from these processes from the total number of observed pretag events. The remaining events are either from $W+$ jets or QCD processes. We determine the relative fraction of QCD events $\left(F_{Q C D}\right)$ by fitting the pretag / $E_{T}$ sideband $\left(E_{T}>0 \mathrm{GeV}\right)$. From the fitted template we estimate the amount of QCD in the signal region integrating the total number of events with $\not_{T}>20 \mathrm{GeV}$. The total number of $W+$ jets events is therefore:

$$
N_{W+\text { Jets }}^{\text {Pretag }}=N_{\text {Pretag }} \cdot\left(1-F_{Q C D}\right)-N_{E W K}-N_{T O P}
$$

where $N_{\text {Pretag }}$ is the observed number of pretag events, $N_{E W K}$ is the number of estimated electroweak events, and $N_{T O P}$ is the number of estimated top events. We must distinguish between $W+$ light and $W+$ heavy flavor since we will eventually $b$-tag the events and light and heavy flavor jets have a different tag rates. The amount of $W+$ heavy flavor is estimated from the relative amount of $W+$ heavy flavor in $W+$ jets Monte Carlo $\left(F_{H F}\right)$, calibrated for the observed heavy fraction in $W+1$ jet data by a correction factor $K$. $W$ theavy flavor events in the pretag sample have a probability to be tagged that is equal to the heavy flavor tagging efficiency. $W+$ light flavor events 
in the pretag sample have a probability to be tagged that is equal to the rate of of false tags (mistags). The amount of $W+$ jets in the tagged sample is therefore

$$
\begin{aligned}
& N_{W+H F}^{\text {tagged }}=N_{W+\text { jets }}^{\text {pretag }} \cdot\left(F_{H F} \cdot K\right) \cdot \epsilon_{t a g} \\
& N_{W+L F}^{\text {tagged }}=N_{W+\text { jets }}^{\text {pretag }} \cdot\left(1-F_{H F} \cdot K\right) \cdot \epsilon_{\text {mistag }}
\end{aligned}
$$

Top and electroweak backgrounds contributions to the tagged sample are estimated using the same Monte Carlo technique as the pretag sample, but with an event detection efficiency that incorporates the tagging efficiency.

\subsection{Non- $W$ QCD Multi-jet}

QCD multi-jet events can fake a $W$ signature when a jet fakes a lepton and overall mismeasurement leads to fake $\mathbb{E}_{T}$. It is difficult to identify the precise sources of mismeasurement and handle them appropriately in a detector simulation. The difficulty is increased by the large number of processes that contribute to the composition of the QCD background at unknown relative rates. We use QCD events taken from a data sideband enriched in QCD to simplify our modeling. Each lepton category is susceptible to particular kinds of fakes. We use different QCD models for central lepton triggered events, and isolated track events. We model central lepton triggered QCD fakes using events where a jet fired the electron trigger, passed the electron kinematic cuts, but failed exactly two of the calorimeter or tracking quality cuts. Events that fail these cuts will have the kinematic properties of $W$-like events, including isolation, but the sample will be enriched in fakes. We call these events "anti-electrons". For an anti-electron to fake a lepton in a given sub-detector, it must fall within the $\eta$ range of that sub-detector. 

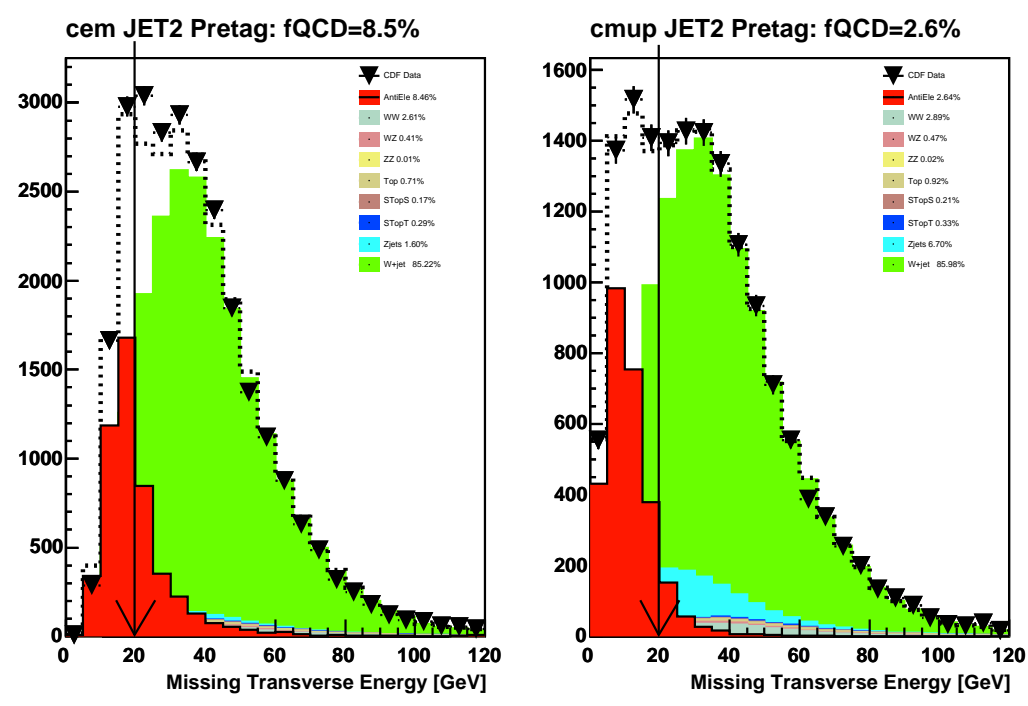

Figure 6.1: QCD fraction estimate for pretag CEM, CMUP, events with two jets. The QCD fraction is shown above the plot.

We model QCD events that fake an isolated track using events recorded on the $\mathbb{E}_{T}+2$ Jets trigger. We require our QCD events to fail the isolation requirement. Lepton isolation is defined as the ratio of calorimeter energy surrounding the lepton $(\Delta R=0.4)$ to the energy of the lepton. Non-isolated leptons are unlikely to come from the decay of an on-shell $W$, and thus are enriched in fakes. We also require that the non-isolated muons fall within the isolated track $\eta$ region $(|\eta|<1.2)$.

We estimate the amount of pretag QCD by fitting the $\not_{T}$ spectrum in pretag data. The fit includes the sideband region below the analysis $\mathbb{E}_{T}$ cut, which is enriched in QCD fakes. The sideband is $\not_{T}>0 \mathrm{GeV}$ for isolated tracks and central leptons. The plug electron sideband is $\mathbb{E}_{T}>15 \mathrm{GeV}$. Figures 6.1 and 6.2 show the pretag $\mathbb{E}_{T}$ fit for CEM, CMUP, CMX, and ISOTRK events. Table 6.1 lists the QCD fractions in for all tag categories. The PHX QCD fits can be found in [16]. 


\begin{tabular}{|l|c|}
\hline Lepton Type & QCD fraction \\
\hline \multicolumn{2}{|c|}{ Pretag } \\
CEM & $0.085 \pm 0.034$ \\
CMUP & $0.026 \pm 0.01$ \\
CMX & $0.031 \pm 0.01$ \\
ISOTRK & $0.18 \pm 0.072$ \\
\hline \multicolumn{2}{|c|}{ One Tag } \\
\hline CEM & $0.108 \pm 0.042$ \\
CMUP & $0.033 \pm 0.013$ \\
CMX & $0.043 \pm 0.017$ \\
ISOTRK & $0.178 \pm 0.071$ \\
\hline \multicolumn{2}{|c|}{ Two SECVTX Tags } \\
\hline CEM & $0.093 \pm 0.037$ \\
CMUP & $0.075 \pm 0.030$ \\
CMX & $0.015 \pm 0.006$ \\
ISOTRK & $0.074 \pm 0.03$ \\
\hline One SECVTX Tag, One JetProb Tag \\
\hline CEM & $0.152 \pm 0.061$ \\
CMUP & $0.031 \pm 0.012$ \\
CMX & $0.032 \pm 0.013$ \\
ISOTRK & $0.098 \pm 0.039$ \\
\hline
\end{tabular}

Table 6.1: QCD fractions for CEM, CMUP, CMX, and ISOTRK events. The fractions are measured separately in each sample. 

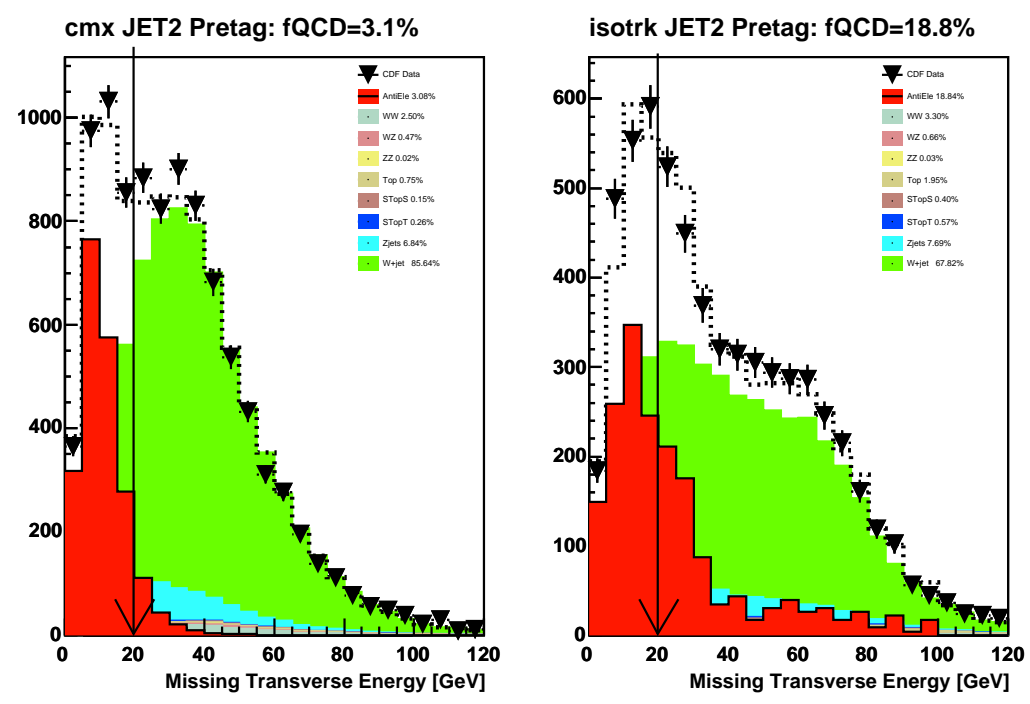

Figure 6.2: QCD fraction estimate for pretag CMX, ISOTRK events with two jets. The QCD fraction is shown above the plot.

The fit has one fixed component and two templates whose normalizations can float. The fixed component is a combination of electroweak processes such as top pair production and diboson production. The normalization of each electroweak template is determined from the theoretical cross section and the Monte Carlo event detection efficiency. The two floating templates are a Monte Carlo $W+$ jets template and a non- $W$ template. The non- $W$ template has a $\mathbb{E}_{T}$ spectrum that peaks near low $\mathbb{E}_{T}$, and it's fitted normalization is driven by the low $E_{T}$ bins. The fitted normalization of the $W+$ jets template is driven by the high $\#_{T}$ region. We use the fractions measured with the $\not_{T}$ sideband to estimate the amount of non- $W$ in the signal region using the following relation: 


$$
N_{Q C D}=F_{Q C D} \cdot N_{\text {PretagData }}=\frac{N_{Q C D}(M E T>20)}{N_{W+\text { Jets }}(M E T>20)} \cdot N_{\text {PretagData }}
$$

where $N_{Q C D}(M E T>20)$ and $N_{W+\text { Jets }}(M E T>20)$ are the respective fitted numbers of QCD and $W+$ jet events above the $\mathbb{E}_{T}$ cut. For the plug electrons, we use the same relation with a $\#_{T}$ cut at $25 \mathrm{GeV}$.

We estimate the uncertainty of the QCD normalization by studying the change in the QCD fraction due to changes in histogram binning, $\mathbb{E}_{T}$ cut, and changes in the QCD model. The alternate QCD model uses non-isolated leptons instead of anti-electrons. Based on these studies, we assign a $40 \%$ uncertainty to the QCD normalization. Although this uncertainty is large, the overall amount of QCD events in our sample is small, and the large uncertainty ultimately has a small impact on our sensitivity.

\subsubsection{QCD Fraction in the Isolated Track Sample}

Isolated track events are potentially susceptible to high QCD contamination because of their relaxed lepton identification requirements. We have seen that the fitted QCD fractions for isolated tracks are larger than other lepton categories from figures 6.1 and 6.2. Specifically, the pretag isolated track QCD fraction is 19\%, which is twice as large as the CEM (9\%), and much larger than the muon samples ( $\sim 3 \%)$. Although the isolated track QCD fraction is larger than other samples, it is not prohibitive, especially after $b$-tagging, where the QCD fractions are approximately $7-10 \%$.

CEM electrons have a very strict QCD veto compared to CMUP or CMX muons. The CEM veto, listed in table 5.2 , has a $M_{T}>20 \mathrm{GeV}$ cut and a cut on $M E T_{\text {sig }}$, while the CMUP and CMX vetos only require $M_{T}>10 \mathrm{GeV}$. To understand how 

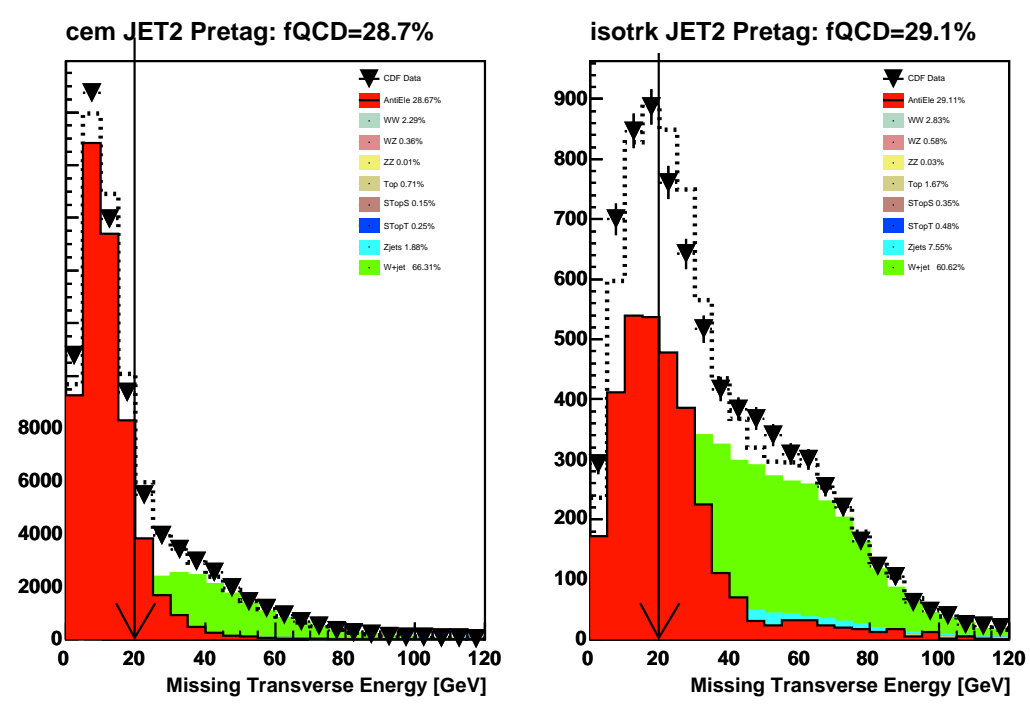

Figure 6.3: QCD fraction estimate for pretag CEM, ISOTRK events with no kinematic QCD vetos applied. The QCD fractions in the regions above $E_{T}>20 \mathrm{GeV}$ for the two samples are comparable.

the lepton identification impacts the QCD fraction, we compare CEM and isolated fraction without a kinematic veto. Figure 6.3 shows the QCD fits of the pretag isolated track and CEM samples without applying kinematic QCD vetos. Note that the isolated track $\mathbb{E}_{T}$ distribution has a different shape than the CEM distribution. The difference in shape is due to the sculpting of the ISOTRK distribution by the $E_{T}+2$ jets trigger requirements. The fitted fraction in the CEM is $0.287 \pm 0.115$, and the fitted fraction for isolated tracks is $0.291 \pm 0.116$. Both QCD fractions are comparable, and agree within their uncertainties. The close matching of the QCD fractions suggests that we will be able to use similar methodology to analyze both samples. 


\subsection{Mistagged Jets}

$W+$ light flavor events with a fake $b$-tag migrate into our signal region. We estimate the number of falsely tagged $W+$ light flavor events using the amount of pretag $W+$ light flavor events and a fake tag rate, called the mistag rate. The mistag rate for each algorithm comes from negatively tagged generic light jet data. False tags that come from tracking resolution or mismeasurement have an equal chance of being positive or negative. This symmetry allows the calibration of the false tag rate using negative tags. There are factors that complicate the calibration of the mistag rate. False tags due to detector material interaction give a positive bias to the overall false tag rate. A further complication of using negative tags is contamination from true b-decays where detector resolution effects create a negative decay length. We account for both the material interaction bias and true $b$ contamination of the fake sample in our calibration. We parameterize the per-jet mistag tag rate for SECVTX in bins of $\eta$, number of vertices, jet $E_{T}$, track multiplicity, and the scalar sum of the total event $E_{T}$ [27]. The Jet Probability mistag parameterization uses the same variables and the SECVTX parameterization and adds the $\mathrm{z}$ position of primary vertex. We must produce a overall event mistag probability that accounts for the probability of mistagging multiple jets in a single event. We calculate the $W+$ jets overall mistag rate, $\epsilon_{\text {mistag }}$, by summing all the event mistag probabilities. We obtain the amount of mistagged $W+$ light flavor events by scaling the amount of pretag $W+$ jets events by according to the mistag rate,

$$
N_{W+L F}^{\text {mistagged }}=N_{W+\text { jets }}^{\text {pretag }} \cdot\left(1-F_{H F} \cdot K\right) \cdot \epsilon_{\text {mistag }}
$$


We estimate the per-jet mistag uncertainty estimate by checking for consistency between the number of expected and observed negative tags in light jet data. The analysis incorporates the uncertainties by fluctuating the per jet tag rates $\pm 1 \sigma$. We use the fluctuated per-jet mistag rates to calculate an overall event weight that accounts for the chance to mistag each jet in the event. We combined the fluctuated event rates into a shifted $\epsilon_{\text {mistag }}$. The new $\epsilon_{\text {mistag }}$ produces a shifted mistag normalization, and the difference between the shifted and the nominal is the uncertainty.

\section{$6.3 W+$ Heavy Flavor}

We refer to $W+b \bar{b}, W+c \bar{c}$, and $W+c q$ collectively as $W+$ heavy flavor. The processes are a source of jets that are likely to have true $b$-tags. The estimation of $W$ + heavy flavor events in the pretag $W+$ jets sample uses a corrected fraction of $W+$ heavy flavor events in ALPGEN $W+$ jets Monte Carlo samples [28]. The fraction of $W+$ heavy flavor events has been studied extensively $[29,30]$. We apply a correction factor $K=1.4 \pm 0.4$ to the heavy flavor fraction $\left(F_{H F}\right)$ in order to calibrate it to the observed heavy flavor rates in $W+1$ jet data.

After estimating the fraction of heavy flavor events in the pretag sample, we estimate an efficiency for those events to enter our tagged sample, $\epsilon_{t a g}$. Monte Carlo mis-modeling of tag efficiencies complicates the calculation of $\epsilon_{\text {tag }}$. Each jet has a probability of being tagged, and the total tag weight for the event is related to the tag probability for each jet. We use Monte Carlo truth information to ensure proper handling of potential overlap between light and heavy flavor Monte Carlo samples. Tagged jets matched to $b$ or $c$ quarks have a probability of being tagged equal to the scale factor. Untagged jets matched to light flavor partons have a probability of being 


\begin{tabular}{ccccc}
\hline \hline Jet Multiplicity & 2 jets & 3 jets & 4 jets & 5 jets \\
\hline$W b \bar{b}(1 \mathrm{~B})(\%)$ & 2.18 & 3.54 & 4.63 & 5.53 \\
$W b \bar{b}(2 \mathrm{~B})(\%)$ & 1.32 & 2.62 & 4.17 & 6.04 \\
$W c \bar{c}(1 \mathrm{C})(\%)$ & 11.04 & 13.96 & 15.18 & 15.84 \\
$W c \bar{c}(2 \mathrm{C})(\%)$ & 2.10 & 4.65 & 7.69 & 10.94 \\
\hline \hline
\end{tabular}

Table 6.2: The heavy-flavor fractions, given in percent, for the $W+$ jets sample. The results from ALPGEN Monte Carlo have been scaled by the data-derived calibration factor of $1.4 \pm 0.4$.

tagged equal to the mistag rate. We use the individual jet tag rates to calculate total event tag probability $P_{\text {event }}^{\text {tag }}$. For instance, the probability for an event to have exactly one tag is:

$$
P_{\text {event }}^{\text {tag }}=1-\prod_{i}^{\text {jets }}\left(1-p_{\text {tag }}^{i}\right),
$$

where $p_{\text {tag }}^{i}$ is either the mistag rate for light flavor jets, or the $b$-tag scale factor for heavy flavor jets. The total sample tag efficiency is

$$
\epsilon_{\text {tag }}=\frac{\sum_{i}^{\text {events }} P_{i}^{\text {tag }}}{N_{\text {pretag }}}
$$

Table 6.2 shows the corrected heavy flavor fractions and total tagging efficiencies for our $W$ +heavy flavor samples. These fractions and efficiencies are used the calculate the over all $W+$ heavy flavor normalizations through the following relation:

$$
N_{W+H F}^{\text {tagged }}=N_{W+j e t s}^{\text {pretag }} \cdot\left(F_{H F} \cdot K\right) \cdot \epsilon_{t a g}
$$




\begin{tabular}{|l|cccc|}
\hline \multicolumn{5}{|c|}{ One SECVTX Tag Efficiency } \\
\hline Jet Multiplicity & 2 jets & 3 jets & 4 jets & 5 jets \\
\hline Event Eff (1B) (\%) & 23.10 & 24.68 & 25.02 & 27.14 \\
Event Eff (2B) (\%) & 30.09 & 30.34 & 30.35 & 29.71 \\
Event Eff (1C) (\%) & 7.02 & 7.69 & 8.68 & 10.24 \\
Event Eff (2C) (\%) & 9.46 & 10.46 & 11.24 & 12.12 \\
\hline \multicolumn{5}{|c|}{ Two SECVTX Tag Efficiency } \\
\hline Jet Multiplicity & 2 jets & 3 jets & 4 jets & 5 jets \\
\hline Event Eff (1B) (\%) & 0.30 & 0.78 & 1.34 & 1.76 \\
Event Eff (2B) (\%) & 8.76 & 9.68 & 10.18 & 11.14 \\
Event Eff (1C) (\%) & 0.04 & 0.12 & 0.24 & 0.40 \\
Event Eff (2C) (\%) & 0.38 & 0.55 & 0.88 & 0.91 \\
\hline One SECVTX TAG + One JETPROB Tag Efficiency \\
\hline Jet Multiplicity & 2 jets & 3 jets & 4 jets & 5 jets \\
\hline Event Eff (1B) (\%) & 0.79 & 1.75 & 2.57 & 3.74 \\
Event Eff (2B) (\%) & 6.95 & 7.78 & 8.86 & 9.77 \\
Event Eff (1C) (\%) & 0.20 & 0.47 & 0.78 & 1.24 \\
Event Eff (2C) (\%) & 1.19 & 1.59 & 2.14 & 2.43 \\
\hline
\end{tabular}

Table 6.3: The table lists the efficiencies for having a either one SECVTX tag, two SECVTX tags, or one SECVTX tag and one Jet Probability tag for various jet multiplicities and heavy flavor contents. The heavy flavor contents are the number of $b$ or $c$ quarks in the Monte Carlo truth that are angularly matched to jets $(\delta R<0.4)$. The efficiencies listed include the efficiency to tag heavy flavor $(b$ or $c$ ) and the efficiency to mistag light flavor. 


\subsection{Top and Electroweak Backgrounds}

The normalization of the diboson, $Z+$ jets, top pair, and single top backgrounds are based on the theoretical cross sections listed in Table $6.4[31,17,32,33]$. The estimate from theory is well-motivated because the cross sections for most of the processes are

theoretically well-known. $Z+$ jets is the only process where the large corrections to the leading order process give large uncertainties in the theory cross section. The small overlap of $Z+$ jets with the $W+$ jets final state marginalizes the impact of the large uncertainty on the overall sensitivity. We estimate the background contributions using the theory cross sections, luminosity, and the Monte Carlo acceptance. We correct the Monte Carlo acceptance with scale factors for lepton identification, trigger efficiencies, the $z$ vertex cut $(|z|<60 \mathrm{~cm}$ fiduciality), and the $b$-tagging scale factor. The number of expected events from each process is,

$$
N=\sigma \cdot \epsilon \cdot \mathcal{L}_{\text {int }}
$$

where $\epsilon$ is the total detection efficiency corrected by all of the scale factors.

\subsection{Summary of Background Estimate}

We calculate the contributions of all the background processes for the integrated luminosity of each trigger. The estimates are summarized in Tables 6.5 through 6.10. Figures 6.4 through 6.6 present the information from the tables as plots of background compared to data. The search region is the two-jet bin. We use higher jet multiplicities as control regions to verify that our background estimate is in good agreement with the data. 


\begin{tabular}{cc}
\hline \hline Theoretical Cross Sections & \\
\hline$W W$ & $12.40 \pm 0.80 \mathrm{pb}$ \\
$W Z$ & $3.96 \pm 0.06 \mathrm{pb}$ \\
$Z Z$ & $1.58 \pm 0.05 \mathrm{pb}$ \\
Single top $s$-channel & $0.88 \pm 0.11 \mathrm{pb}$ \\
Single top $t$-channel & $1.98 \pm 0.25 \mathrm{pb}$ \\
$t \bar{t}$ & $6.7 \pm 0.83 \mathrm{pb}$ \\
$Z+$ Jets & $787.4 \pm 85$ \\
\hline \hline
\end{tabular}

Table 6.4: Theoretical cross sections and uncertainties for the electroweak and top backgrounds. Top cross sections assume a mass of $m_{t}=175 \mathrm{GeV} / c^{2}$.

\begin{tabular}{|c|c|c|c|c|}
\hline \multicolumn{5}{|c|}{ CDF Run II Preliminary $2.7 \mathrm{fb}^{-1}$} \\
\multicolumn{5}{|c|}{ Tight Lepton Background Predication and Event Yields } \\
\multicolumn{5}{|c|}{ Exactly One Secvtx Tag Events } \\
\hline Process & 2jets & 3jets & 4jets & 5jets \\
\hline \hline All Pretag Candidates & 38729 & 6380 & 1677 & 386 \\
WW & $41 \pm 4.2$ & $12 \pm 1.2$ & $2.9 \pm 0.25$ & $0.71 \pm 0.055$ \\
WZ & $14 \pm 0.94$ & $3.4 \pm 0.23$ & $0.93 \pm 0.064$ & $0.20 \pm 0.015$ \\
ZZ & $0.48 \pm 0.037$ & $0.19 \pm 0.015$ & $0.081 \pm 0.0070$ & $0.023 \pm 0.0020$ \\
Top Pair Prod & $100 \pm 14$ & $190 \pm 26$ & $180 \pm 26$ & $59 \pm 8.8$ \\
Single Top s-Channel & $24 \pm 2.2$ & $6.9 \pm 0.67$ & $1.5 \pm 0.15$ & $0.28 \pm 0.030$ \\
Single Top t-Channel & $43 \pm 4.4$ & $9.2 \pm 0.94$ & $1.6 \pm 0.17$ & $0.22 \pm 0.024$ \\
Z+Jets & $29 \pm 3.4$ & $8.7 \pm 0.96$ & $2.7 \pm 0.29$ & $0.52 \pm 0.056$ \\
W+bottom & $370 \pm 111$ & $91 \pm 27$ & $19 \pm 5.7$ & $4.0 \pm 1.2$ \\
W+charm & $360 \pm 108$ & $81 \pm 24$ & $17 \pm 5.1$ & $3.6 \pm 1.1$ \\
Mistags & $320 \pm 42$ & $84 \pm 13$ & $19 \pm 5.1$ & $3.8 \pm 1.5$ \\
Non-W & $110 \pm 43$ & $40 \pm 17$ & $17 \pm 14$ & $4.5 \pm 4.4$ \\
\hline Total Prediction & $1400 \pm 290$ & $530 \pm 75$ & $270 \pm 34$ & $77 \pm 11$ \\
Observed & 1404 & 486 & 281 & 81 \\
\hline
\end{tabular}

Table 6.5: Background summary table for central leptons with one secondary vertex tag. The hashed region indicates the uncertainty on the background estimate. 


\begin{tabular}{|c|c|c|c|c|}
\hline \multicolumn{5}{|c|}{ CDF Run II Preliminary 2.7 $\mathrm{fb}^{-1}$} \\
& Tight Lepton Background Predication and Event Yields \\
& One Secvtx Tag, One Jet Prob Tag Events \\
\hline Process & 2jets & 3jets & 4jets & 5jets \\
\hline \hline All Pretag Candidates & 44723 & 7573 & 1677 & 386 \\
WW & $1.2 \pm 0.53$ & $0.85 \pm 0.31$ & $0.40 \pm 0.13$ & $0.16 \pm 0.047$ \\
WZ & $2.5 \pm 0.42$ & $0.78 \pm 0.16$ & $0.18 \pm 0.043$ & $0.052 \pm 0.013$ \\
ZZ & $0.098 \pm 0.017$ & $0.053 \pm 0.0090$ & $0.021 \pm 0.0040$ & $0.0050 \pm 0.0010$ \\
Top Pair Prod & $20 \pm 4.2$ & $64 \pm 13$ & $79 \pm 16$ & $30 \pm 6.1$ \\
Single Top s-Channel & $7.0 \pm 1.1$ & $2.5 \pm 0.42$ & $0.57 \pm 0.10$ & $0.13 \pm 0.024$ \\
Single Top t-Channel & $2.1 \pm 0.64$ & $1.7 \pm 0.36$ & $0.46 \pm 0.091$ & $0.076 \pm 0.015$ \\
Z+Jets & $1.8 \pm 0.54$ & $1.2 \pm 0.35$ & $0.34 \pm 0.12$ & $0.097 \pm 0.032$ \\
W+bottom & $49 \pm 15$ & $17 \pm 5.1$ & $4.9 \pm 1.5$ & $1.3 \pm 0.4$ \\
W+charm & $18 \pm 5.4$ & $7.9 \pm 2.4$ & $2.6 \pm 0.8$ & $0.67 \pm 0.19$ \\
Mistags & $5.8 \pm 6.0$ & $3.0 \pm 3.4$ & $1.0 \pm 1.0$ & $0.29 \pm 0.37$ \\
Non-W & $11 \pm 5.3$ & $6.6 \pm 3.5$ & $3.4 \pm 3.4$ & $1.5 \pm 2.1$ \\
\hline Total Prediction & $120 \pm 30$ & $110 \pm 19$ & $93 \pm 17$ & $34 \pm 6.6$ \\
Observed & 126 & 108 & 102 & 36 \\
\hline
\end{tabular}

Table 6.6: Background summary table for central leptons with one secondary vertex tag and one jet probability tag. The hashed region indicates the uncertainty on the background estimate.

\begin{tabular}{|c|c|c|c|c|}
\hline \multicolumn{5}{|c|}{ CDF Run II Preliminary 2.7 $\mathrm{fb}^{-1}$} \\
\multicolumn{5}{|c|}{ Tight Lepton Background Predication and Event Yields } \\
Two Secvtx Tag Events \\
\hline Process & 2jets & 3jets & 4jets & 5jets \\
\hline \hline All Pretag Candidates & 44723 & 7573 & 1677 & 386 \\
WZ & $3.3 \pm 0.37$ & $0.94 \pm 0.11$ & $0.19 \pm 0.024$ & $0.039 \pm 0.0050$ \\
ZZ & $0.099 \pm 0.011$ & $0.073 \pm 0.0080$ & $0.019 \pm 0.0020$ & $0.0050 \pm 0.0010$ \\
Top Pair Prod & $26 \pm 4.2$ & $77 \pm 12$ & $100 \pm 16$ & $36 \pm 5.9$ \\
Single Top s-Channel & $9.6 \pm 1.2$ & $3.3 \pm 0.41$ & $0.72 \pm 0.092$ & $0.15 \pm 0.020$ \\
Single Top t-Channel & $2.2 \pm 0.30$ & $1.9 \pm 0.26$ & $0.53 \pm 0.072$ & $0.10 \pm 0.014$ \\
Z+Jets & $1.4 \pm 0.20$ & $0.95 \pm 0.13$ & $0.26 \pm 0.038$ & $0.085 \pm 0.013$ \\
W+bottom & $55 \pm 16.5$ & $18 \pm 5.4$ & $4.9 \pm 1.5$ & $1.2 \pm 0.36$ \\
W+charm & $4.9 \pm 1.5$ & $2.4 \pm 0.72$ & $0.94 \pm 0.28$ & $0.25 \pm 0.08$ \\
Mistags & $1.4 \pm 0.39$ & $0.93 \pm 0.30$ & $0.34 \pm 0.12$ & $0.10 \pm 0.047$ \\
Non-W & $9.0 \pm 4.0$ & $5.0 \pm 2.0$ & $0.74 \pm 1.6$ & $0.23 \pm 1.5$ \\
\hline Total Prediction & $110 \pm 25$ & $110 \pm 16$ & $110 \pm 17$ & $38 \pm 6.1$ \\
Observed & 114 & 132 & 104 & 42 \\
\hline
\end{tabular}

Table 6.7: Background summary table for central leptons with two secondary vertex tags. The hashed region indicates the uncertainty on the background estimate. 


\begin{tabular}{|c|c|c|c|c|}
\hline \multicolumn{5}{|c|}{ CDF Run II Preliminary 2.7 $\mathrm{fb}^{-1}$} \\
\multicolumn{5}{|c|}{ Isolated Track Background Predication and Event Yields } \\
\hline \multicolumn{7}{|c|}{ Exactly One Secvtx Tag Events } \\
\hline Process & 2jets & 3jets & 4jets & jjets \\
\hline \hline All Pretag Candidates & 4253 & 1380 & 427 & 117 \\
WW & $6.4 \pm 0.65$ & $2.8 \pm 0.25$ & $0.75 \pm 0.066$ & $0.23 \pm 0.019$ \\
WZ & $2.4 \pm 0.16$ & $0.92 \pm 0.061$ & $0.19 \pm 0.013$ & $0.063 \pm 0.0050$ \\
ZZ & $0.13 \pm 0.0090$ & $0.052 \pm 0.0040$ & $0.0070 \pm 0.0010$ & $0.0060 \pm 0.0010$ \\
Top Pair Prod & $28 \pm 3.8$ & $58 \pm 8.0$ & $53 \pm 7.6$ & $17 \pm 2.5$ \\
Single Top s-Channel & $6.1 \pm 0.58$ & $1.9 \pm 0.19$ & $0.43 \pm 0.044$ & $0.078 \pm 0.010$ \\
Single Top t-Channel & $10 \pm 1.0$ & $2.3 \pm 0.24$ & $0.41 \pm 0.045$ & $0.068 \pm 0.0070$ \\
Z+Jets & $9.1 \pm 1.0$ & $3.3 \pm 0.36$ & $0.74 \pm 0.077$ & $0.16 \pm 0.017$ \\
W+bottom & $40 \pm 12$ & $18 \pm 5.4$ & $5.3 \pm 1.59$ & $1.9 \pm .57$ \\
W+charm & $37 \pm 11.1$ & $16 \pm 4.8$ & $4.7 \pm 1.41$ & $1.5 \pm 0.45$ \\
Mistags & $43 \pm 5.6$ & $18 \pm 2.34$ & $4.8 \pm 0.62$ & $1.8 \pm 0.23$ \\
Non-W & $38 \pm 15$ & $22 \pm 8.9$ & $5.3 \pm 4.2$ & $2.1 \pm 1.7$ \\
\hline Total Prediction & $220 \pm 35$ & $140 \pm 19$ & $76 \pm 9.9$ & $25 \pm 3.4$ \\
Observed & 208 & 150 & 78 & 31 \\
\hline
\end{tabular}

Table 6.8: Background summary table for isolated tracks with one secondary vertex tag. The hashed region indicates the uncertainty on the background estimate.

CDF Run II Preliminary $2.7 \mathrm{fb}^{-1}$

Isolated Track Background Predication and Event Yields

One Secvtx Tag, One Jet Prob Tag Events

\begin{tabular}{|c|c|c|c|c|}
\hline Process & 2jets & 3jets & 4jets & 5jets \\
\hline \hline All Pretag Candidates & 5149 & 1623 & 487 & 124 \\
WW & $0.20 \pm 0.091$ & $0.24 \pm 0.092$ & $0.10 \pm 0.030$ & $0.030 \pm 0.010$ \\
WZ & $0.51 \pm 0.090$ & $0.20 \pm 0.041$ & $0.048 \pm 0.010$ & $0.013 \pm 0.0040$ \\
ZZ & $0.032 \pm 0.0060$ & $0.021 \pm 0.0050$ & $0.0070 \pm 0.0010$ & $0.0020 \pm 0.0010$ \\
Top Pair Prod & $6.4 \pm 1.3$ & $20 \pm 4.2$ & $25 \pm 4.9$ & $9.0 \pm 1.8$ \\
Single Top s-Channel & $1.9 \pm 0.31$ & $0.74 \pm 0.12$ & $0.19 \pm 0.034$ & $0.043 \pm 0.0090$ \\
Single Top t-Channel & $0.53 \pm 0.16$ & $0.50 \pm 0.11$ & $0.12 \pm 0.025$ & $0.028 \pm 0.0050$ \\
Z+Jets & $0.61 \pm 0.20$ & $0.41 \pm 0.13$ & $0.13 \pm 0.044$ & $0.039 \pm 0.013$ \\
W+bottom & $6.0 \pm 1.8$ & $3.4 \pm 1.0$ & $1.4 \pm 0.42$ & $0.59 \pm 0.18$ \\
W+charm & $2.1 \pm 0.63$ & $1.6 \pm 0.48$ & $0.77 \pm 0.23$ & $0.34 \pm 0.10$ \\
Mistags & $0.80 \pm 0.07$ & $0.61 \pm 0.05$ & $0.27 \pm 0.02$ & $0.13 \pm 0.01$ \\
Non-W & $2.0 \pm 0.79$ & $1.4 \pm 0.55$ & $0.99 \pm 0.79$ & $0.37 \pm 0.50$ \\
\hline Total Prediction & $21 \pm 4.4$ & $29 \pm 5.2$ & $29 \pm 5.1$ & $11 \pm 2.0$ \\
Observed & 21 & 30 & 32 & 12 \\
\hline
\end{tabular}

Table 6.9: Background summary table for isolated tracks with one secondary vertex tag and one jet probability tag. The hashed region indicates the uncertainty on the background estimate. 

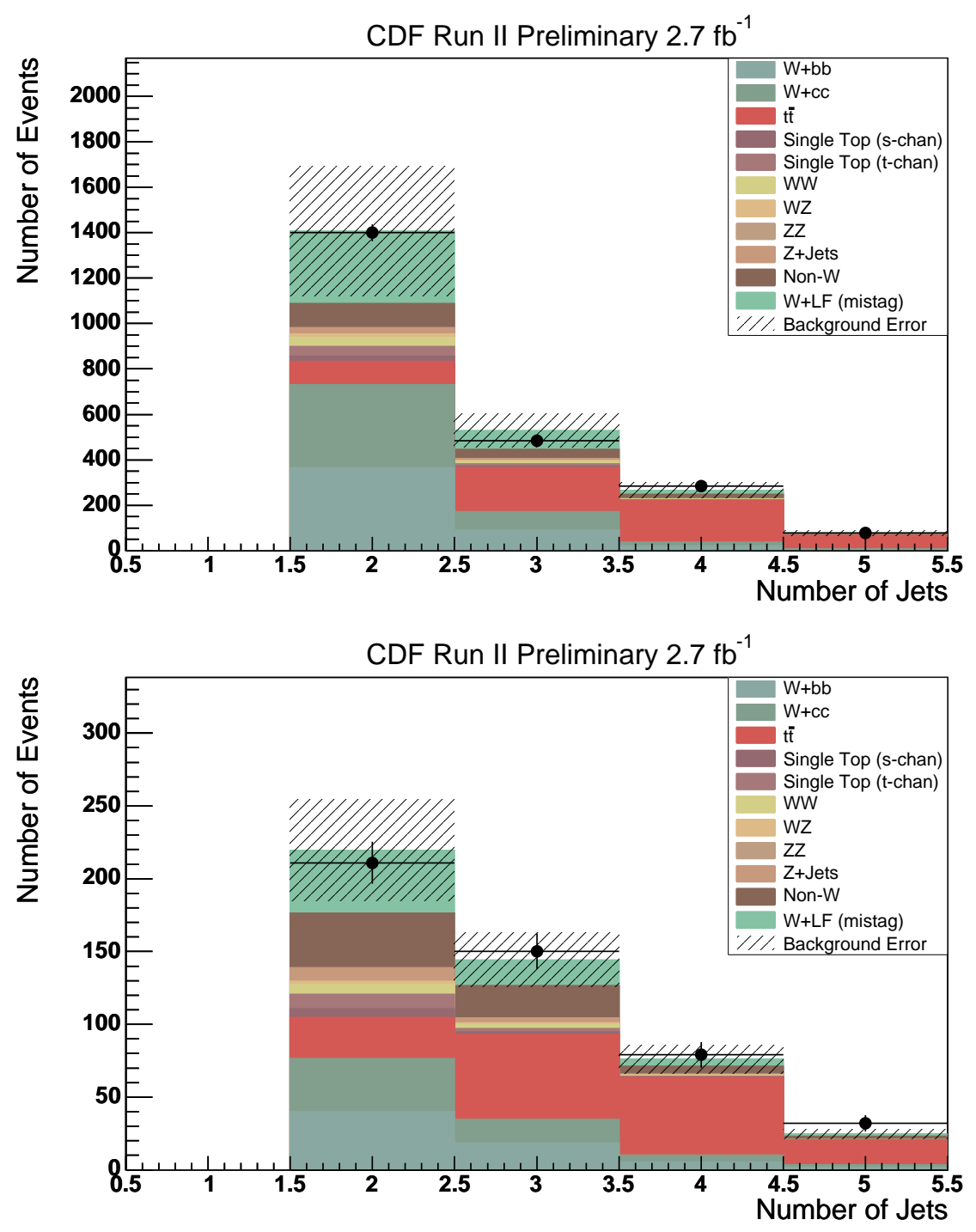

Figure 6.4: Number of expected and observed background events for events with exactly one SECVTX tag, shown as a function of jet multiplicity. The plots show tight leptons (left) and isolated tracks (right). 

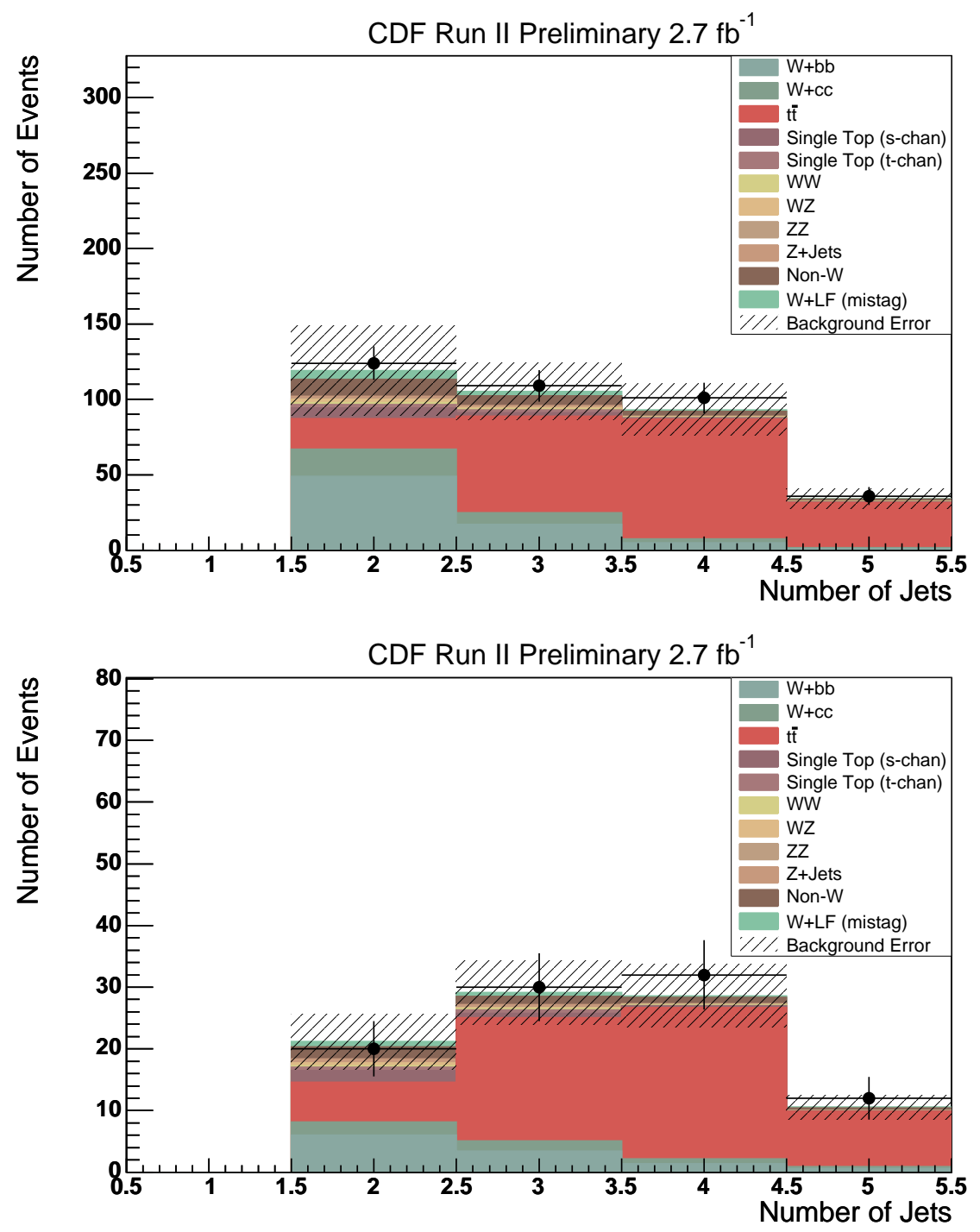

Figure 6.5: Number of expected and observed background events for events with one SECVTX tag and one Jet Probability tag, shown as a function of jet multiplicity. The plots show tight leptons (left) and isolated tracks (right). 

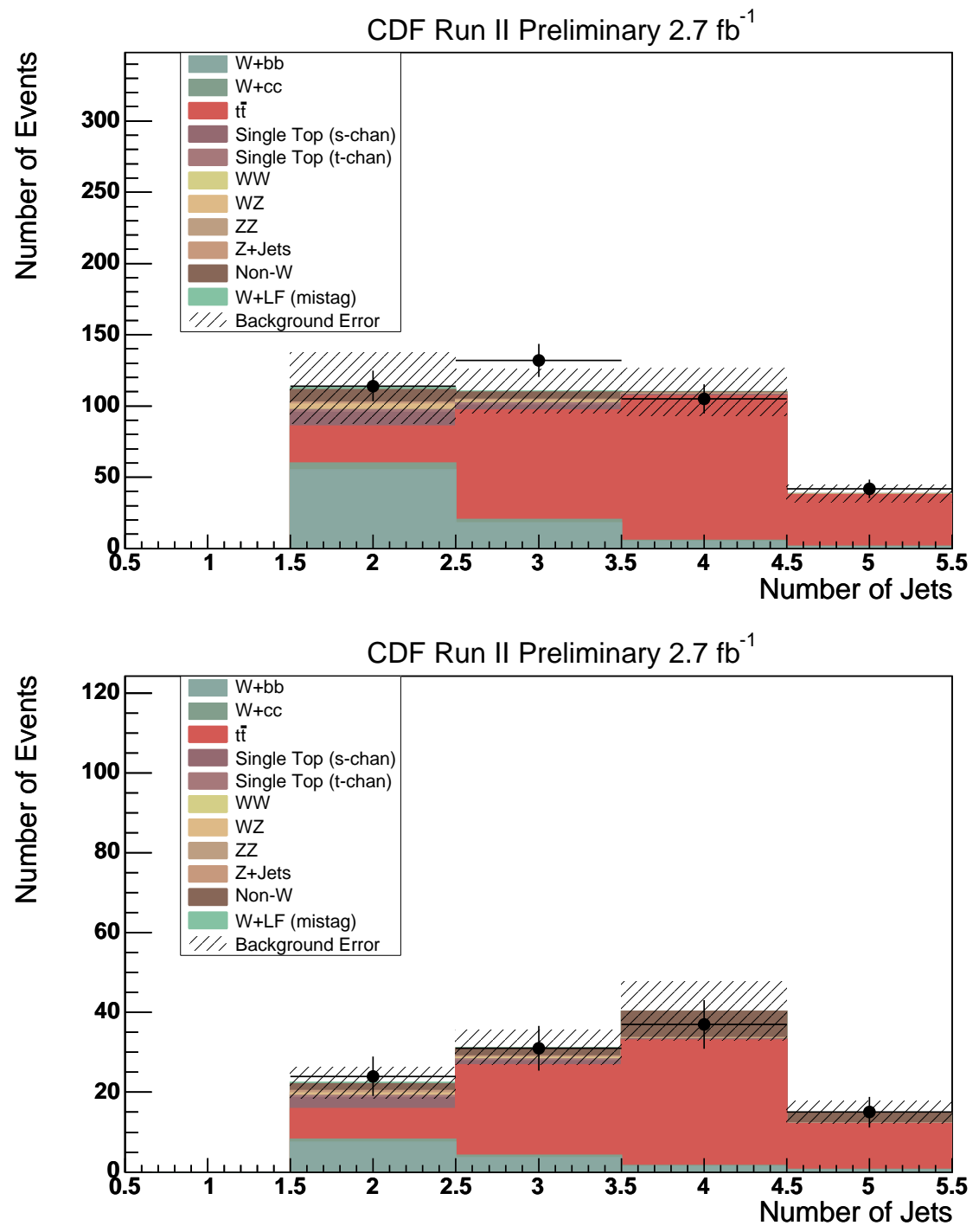

Figure 6.6: Number of expected and observed background events for events with one SECVTX tag and one Jet Probability tag, shown as a function of jet multiplicity. The plots show tight leptons (left) and isolated tracks (right). 


\begin{tabular}{|c|c|c|c|c|}
\hline \multicolumn{5}{|c|}{$\begin{array}{c}\text { CDF Run II Preliminary } 2.7 \mathrm{fb}^{-1} \\
\text { Isolated Track Background Predication and Event Yields } \\
\text { Two Secvtx Tag Events }\end{array}$} \\
\hline Process & 2jets & 3jets & 4 jets & 5 jets \\
\hline \hline All Pretag Candidates & 5149 & 1623 & 487 & 124 \\
WW & $0.036 \pm 0.0080$ & $0.13 \pm 0.021$ & $0.067 \pm 0.012$ & $0.019 \pm 0.0030$ \\
WZ & $0.65 \pm 0.072$ & $0.24 \pm 0.027$ & $0.029 \pm 0.0030$ & $0.010 \pm 0.0010$ \\
ZZ & $0.045 \pm 0.0050$ & $0.025 \pm 0.0030$ & $0.010 \pm 0.0010$ & $0.0020 \pm 0$ \\
Top Pair Prod & $7.7 \pm 1.2$ & $23 \pm 3.7$ & $31 \pm 5.1$ & $11 \pm 1.8$ \\
Single Top s-Channel & $2.7 \pm 0.34$ & $0.91 \pm 0.12$ & $0.21 \pm 0.027$ & $0.045 \pm 0.0060$ \\
Single Top t-Channel & $0.58 \pm 0.080$ & $0.57 \pm 0.077$ & $0.18 \pm 0.024$ & $0.035 \pm 0.0050$ \\
Z+Jets & $0.51 \pm 0.069$ & $0.32 \pm 0.045$ & $0.093 \pm 0.014$ & $0.025 \pm 0.0040$ \\
W+bottom & $7.5 \pm 2.25$ & $3.6 \pm 1.1$ & $1.4 \pm 0.42$ & $0.53 \pm 0.16$ \\
W+charm & $0.68 \pm 0.20$ & $0.56 \pm 0.17$ & $0.26 \pm 0.08$ & $0.12 \pm 0.04$ \\
Mistags & $0.27 \pm 0.02$ & $0.20 \pm 0.02$ & $0.089 \pm 0.01$ & $0.052 \pm 0.01$ \\
Non-W & $1.8 \pm 0.71$ & $1.9 \pm 0.76$ & $6.5 \pm 5.2$ & $2.6 \pm 2.1$ \\
Total Prediction & $22 \pm 4.0$ & $31 \pm 4.3$ & $40 \pm 7.3$ & $15 \pm 2.8$ \\
Observed & 24 & 31 & 37 & 15 \\
\hline
\end{tabular}

Table 6.10: Background summary table for isolated track events with two secondary vertex tags. The hashed region indicates the uncertainty on the background estimate. 


\section{CHAPTER 7}

\section{HIGGS BOSON SIGNAL ACCEPTANCE}

We simulate the $W H$ signal kinematics using the PYTHIA Monte Carlo program [34]. We generated signal Monte Carlo samples for Higgs masses between 100 and $150 \mathrm{GeV} / c^{2}$. The number of expected $W H \rightarrow \ell \nu b \bar{b}$ events $N$ is:

$$
N=\epsilon \cdot \int \mathcal{L} d t \cdot \sigma(p \bar{p} \rightarrow W H) \cdot \mathcal{B}(H \rightarrow b \bar{b})
$$

where $\epsilon, \int \mathcal{L} d t, \sigma(p \bar{p} \rightarrow W H)$, and $\mathcal{B}(H \rightarrow b \bar{b})$ are the event detection efficiency, integrated luminosity, production cross section, and branching ratio, respectively. The production cross section and branching ratio are calculated to NLO precision [?]. We correct the acceptance $\epsilon$ so that the Monte Carlo efficiencies match the observed efficiencies in data. The corrected acceptance is,

$$
\epsilon=\sum_{\ell=e, \mu, \tau}\left(\epsilon_{z_{0}} \cdot \epsilon_{\text {trigger }} \cdot \epsilon_{\text {lepton ID }} \cdot \epsilon_{b \operatorname{tag}} \cdot \epsilon_{\mathrm{acc}} \cdot \mathcal{B}(W \rightarrow \ell \nu)\right)
$$

where $\epsilon_{z_{0}}, \epsilon_{\text {trigger }}, \epsilon_{\text {lepton ID }}, \epsilon_{b t a g}$, and $\epsilon_{\text {acc }}$ are scaled efficiencies to meet the requirements of primary vertex, trigger, lepton identification, $b$-tagging, and pass all of the acceptance cuts. The major sources of inefficiency are the lepton identification, jet kinematics, and $b$-tagging. 


\begin{tabular}{|c|r|}
\hline \multicolumn{2}{|c|}{ CDF Run II Preliminary 2.7 $\mathrm{fb}^{-1}$} \\
Number of Expected WH Events \\
$\left(M_{H}=120 \mathrm{GeV} / \mathrm{c}^{2}\right)$ \\
\hline Detector & Expected Number of WH events \\
\hline & One Tag \\
\hline CEM & 1.580 \\
CMUP & 0.907 \\
CMX & 0.437 \\
ISOTRK & 0.723 \\
\hline Total & 3.647 \\
\hline & ST+ST Tag \\
\hline CEM & 0.656 \\
CMUP & 0.374 \\
CMX & 0.167 \\
ISOTRK & 0.359 \\
\hline Total & 1.556 \\
\hline & ST+JP Tag \\
\hline CEM & 0.476 \\
CMUP & 0.255 \\
CMX & 0.127 \\
ISOTRK & 0.232 \\
\hline Total & 1.09 \\
\hline
\end{tabular}

Table 7.1: Expected number of $\mathrm{WH}$ events at a $\mathrm{M}(\mathrm{H})=120 \mathrm{GeV} / \mathrm{c}^{2}$, shown broken down for tag categories and lepton types 
We estimate the expected number of signal events using Eq. 7.1 at each Higgs boson mass point. Table 7.1 shows the number of expected WH events for $M_{H}=120$ $\mathrm{GeV} / \mathrm{c}^{2}$. The total systematic uncertainty on the acceptance comes from several sources, including the jet energy scale, initial and final state radiation, lepton identification, trigger efficiencies, and $b$-tagging scale factor.

We estimate the impact of changes in initial and final state radiation (ISR, FSR) by halving and doubling the parameters related to ISR and FSR in the Monte Carlo event generation [35]. The systematic uncertainty is the difference between the nominal and the shifted acceptance.

The $W H$ acceptance depends on the proton and anti-proton parton distribution functions (PDFs). We use nominal and 90\% confidence interval PDFs provided by CTEQ6M [36]. We calculate the probability for each $W H$ event generated using nominal PDFs to be produced by the $90 \%$ confidence interval PDFs. We re-calculate the acceptance, weighting each event by the probability to be produced with the shifted PDFs. The uncertainty from PDFs is the difference between the nominal and shifted acceptance [22].

Uncertainties in the jet energy scale (JES) lead to uncertainties both on $W H$ event detection efficiency and kinematic distributions [37]. We account for JES uncertainty by shifting jet energies in $W H$ Monte Carlo samples by $\pm 1 \sigma$. We re-calculate the acceptance with the shifted JES and use the deviation from the nominal acceptance as an uncertainty on the signal rate. The JES uncertainty has a small overall impact on $W H$ and background shapes. We do not add an extra shape systematic for our JES uncertainty, as it is expected to be very small compared with the normalization uncertainties. 


\begin{tabular}{cccc}
\hline \hline Source & \multicolumn{3}{c}{ Uncertainty $(\%)$} \\
\hline & ST+ST & ST+JP & one tag \\
\hline Trigger Lepton (ISOTRK) ID & $\sim 2 \%(8.85 \%)$ & $\sim 2 \%(8.85 \%)$ & $\sim 2 \%(8.85 \%)$ \\
Lepton (MET+Jets) Trigger & $<1 \%(3 \%)$ & $<1 \%(3 \%)$ & $<1 \%(3 \%)$ \\
ISR/FSR & $5.2 \%$ & $4.0 \%$ & $2.9 \%$ \\
PDF & $2.1 \%$ & $1.5 \%$ & $2.3 \%$ \\
JES & $2.5 \%$ & $2.8 \%$ & $1.2 \%$ \\
b-tagging & $8.4 \%$ & $9.1 \%$ & $3.5 \%$ \\
\hline Total (ISOTRK) & $10.6 \%(13.8 \%)$ & $10.5 \%(14.0 \%)$ & $5.6 \%(10.1 \%)$ \\
\hline \hline
\end{tabular}

Table 7.2: Systematic uncertainty on the $W H$ acceptance. "ST+ST" refers to double secondary vertex tagged events while "ST+JP" refers to secondary vertex plus jet probability tagged events.

The systematic uncertainty on the per-jet $b$-tagging efficiency is $4.2 \%$ for SECVTX tags, and $8.2 \%$ for jet probability tags. The overall event uncertainties for each tag category are $3.5 \%$ for events with exactly one SECVTX tag, $8.4 \%$ for events with two SECVTX tags, and $9.1 \%$ for events with one SECVTX and one Jet Probability tag.

Table 7.2 summarizes the uncertainties on $W H$ acceptance. 


\section{CHAPTER 8}

\section{MODELING AND CONTROL REGIONS}

Searching for $W H$ with a counting experiment is challenging because of the low signal to background ratio and the large uncertainties on the background. Even in the samples with relatively high purity, the signal is smaller than the uncertainty on the background. For example, in the double SECVTx tagged isolated track sample, we expect $0.44 \mathrm{WH}$ events and $22 \pm 4$ background events. Looking for an excess in a kinematic distribution can provide an increase in sensitivity beyond a counting experiment. If the kinematic distribution has a region that is enriched in signal, that region will be more sensitive to signal than the overall sample. We use kinematic distributions with high-purity regions in order to improve our Higgs signal sensitivity. Searching for an excess above background requires a reliable background kinematic model. We check our modeling using control regions that are enriched in background not directly used in our measurement. The following chapter focuses on isolated track modeling.

\subsection{Pretag Control Plots}

The pretag sample is the foundation of our background estimate. The dominant backgrounds are non- $W$ QCD and $W+$ jets. Proper modeling in the pretag region 
builds confidence in our extrapolation to the tagged regions. The pretag region's large statistics allow close comparison of the model and the data. Low statistics after tagging can make comparison difficult, so it important to build confidence in a region where statistics are good. We check our modeling of important objects such as the final state lepton, $E_{T}$, and two jets. Figure 8.1 shows the how the background modeling compares to observed pretag distributions for the transverse energy of each of the four objects in the event. The distributions show good agreement between the model and the observed data. Figure 8.3 shows quantities that depend on modeling correlations between event objects. The figure shows the $W$ transverse $\operatorname{mass}\left(M_{T}(W)\right)$ and reconstructed Higgs mass $M(j j+)$. The transverse mass is calculated according to equation 5.2. $M(j j+)$ is the invariant mass of the two jet system after accounting for loose jet activity. If there are additional loose jets in the event $\left(12<E_{T}<20\right.$ GeV or $|\eta|<2.4$ and $\left.E_{T}>12\right)$ that are nearby one of the jets $(\Delta R<0.9)$, then the closest loose jet is added into $M(j j+)$. The good agreement of the $M_{T}$ distribution gives us confidence that our fit in the low $\mathbb{E}_{T}$ region $\left(\mathbb{E}_{T}>0\right)$ miss produced a valid QCD estimate. Figure 8.3 shows good modeling of the $W$ transverse mass and the dijet mass.

\subsection{One Tag Plots}

Statistics in the one tag sample are lower than the pretag sample but still high enough to allow model validation. The increase in the signal to background ratio does not spoil the use of one tag distributions as control regions. Figures 8.4 through 8.5 shows the transverse energy distributions for the event objects, and figure 8.6 shows the $W$ transverse mass. The good agreement between our model and the observed 

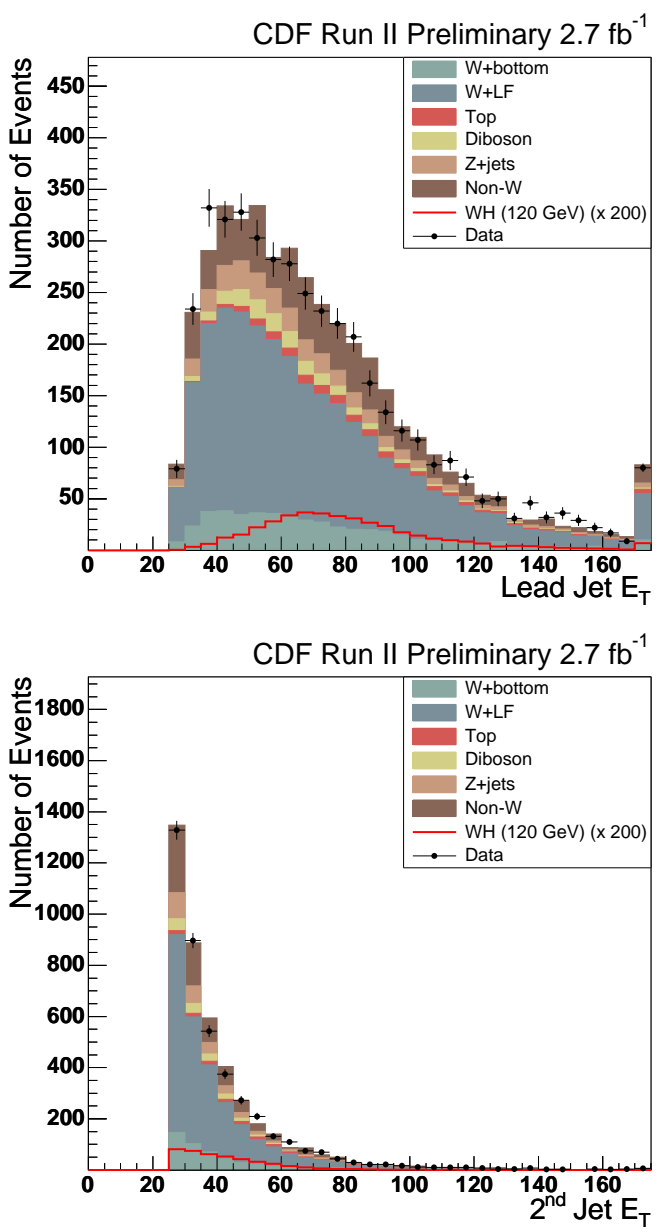

Figure 8.1: Distributions of the lead jet $E_{T}$ and second jet $E_{T}$. for pretag isolated track events. The plots show the $W H$ signal at 200 times it's Standard Model expectation. 

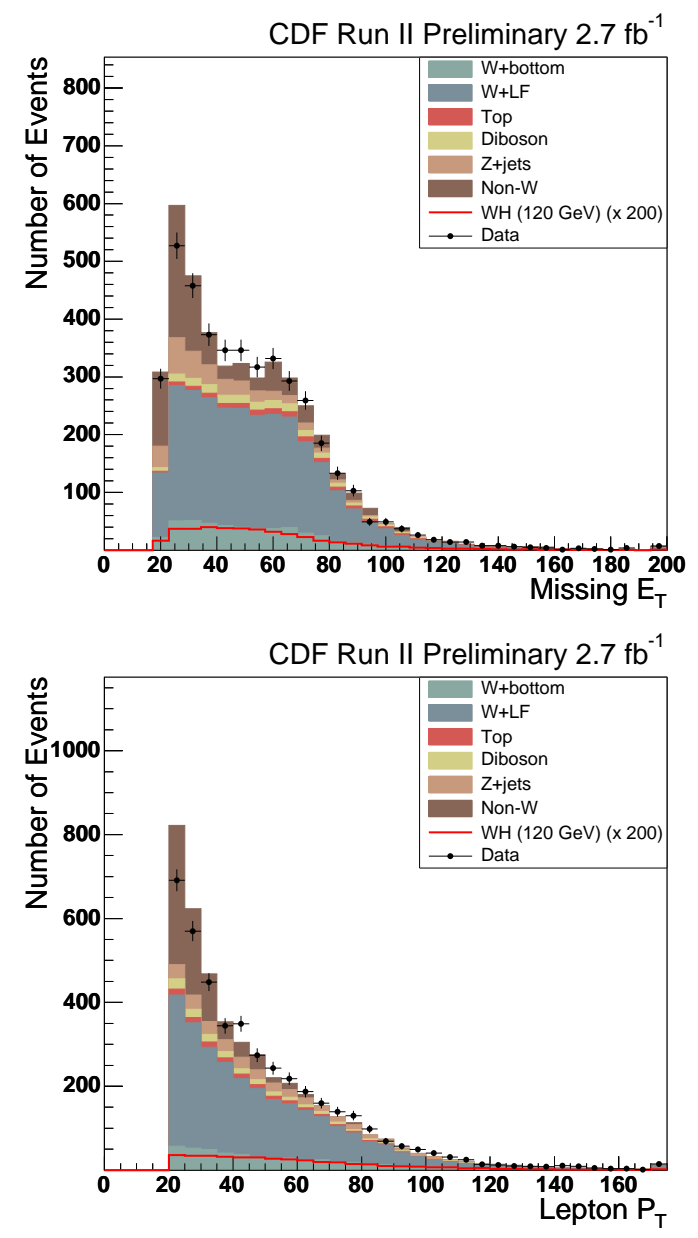

Figure 8.2: Distributions of the $\mathbb{E}_{T}$ and lepton $p_{T}$ for pretag isolated track events. The plots show the WH signal at 200 times it's Standard Model expectation. 

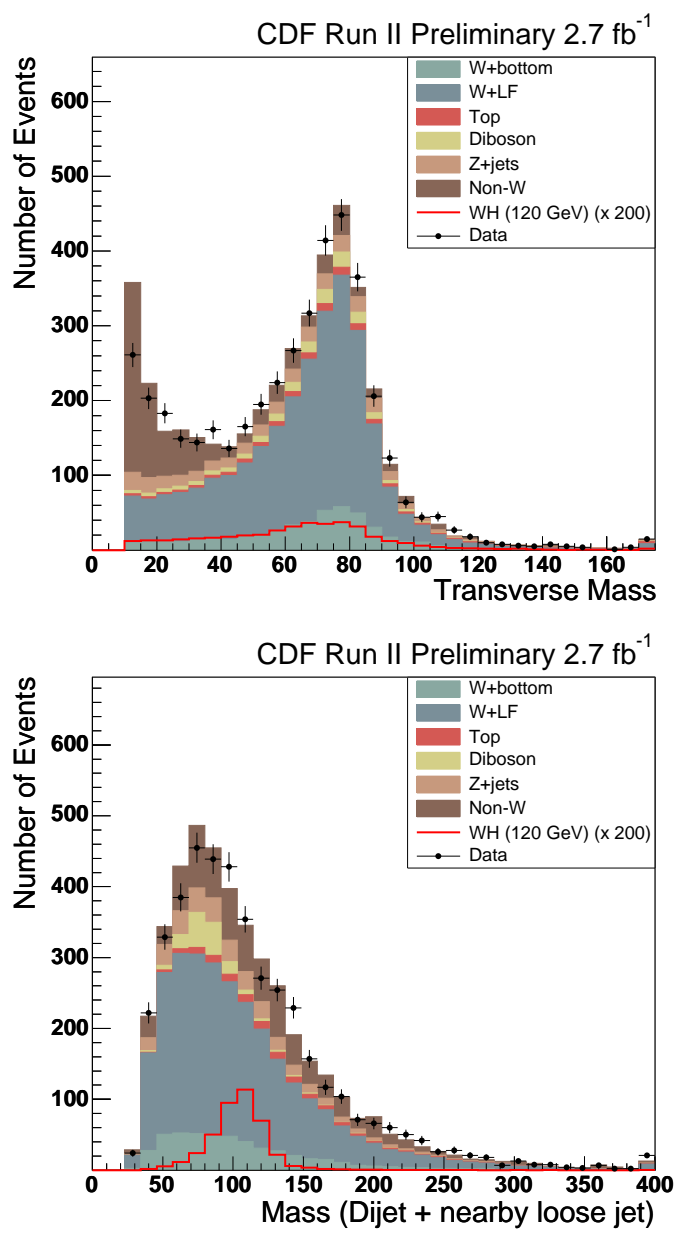

Figure 8.3: Distributions of $M_{T}(W)$ and $M(j j+)$ for pretag isolated track events. The plots show the $W H$ signal at 200 times it's Standard Model expectation. 
data gives us confidence that we have properly extrapolated the backgrounds from the pretag and from the $\mathbb{E}_{T}$ sideband.
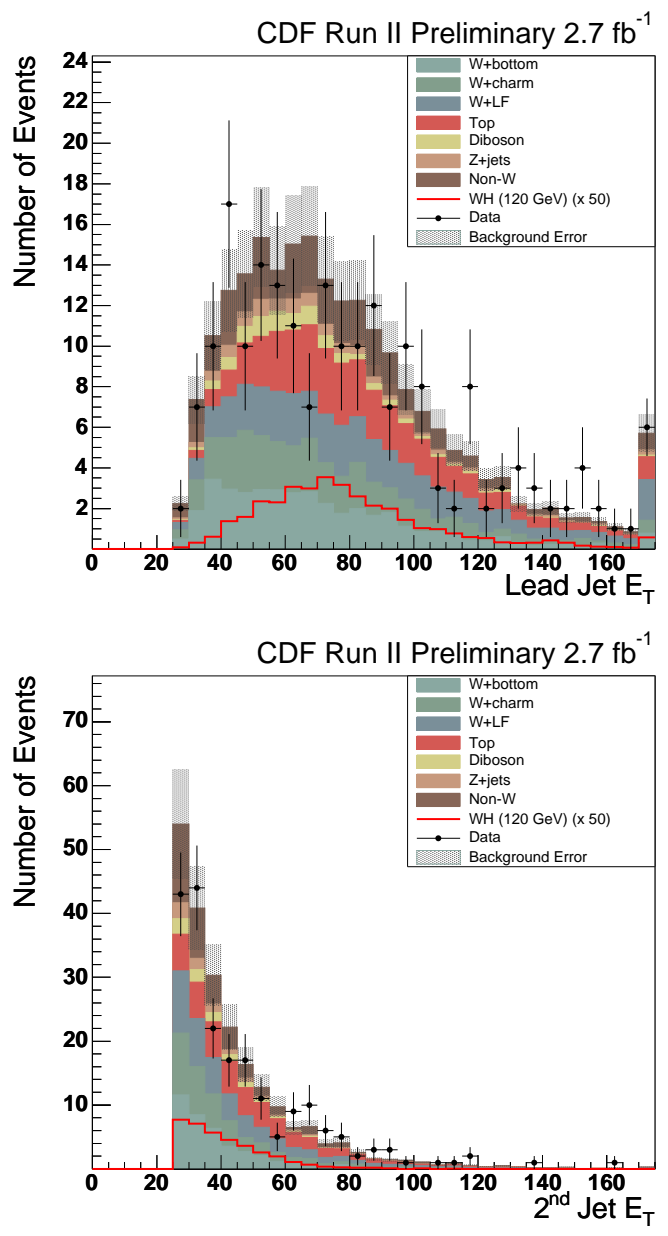

Figure 8.4: Distributions of the lead jet $E_{T}$ and second jet $E_{T}$ for one tag isolated track events. The plots show the $W H$ signal at 50 times it's Standard Model expectation. 

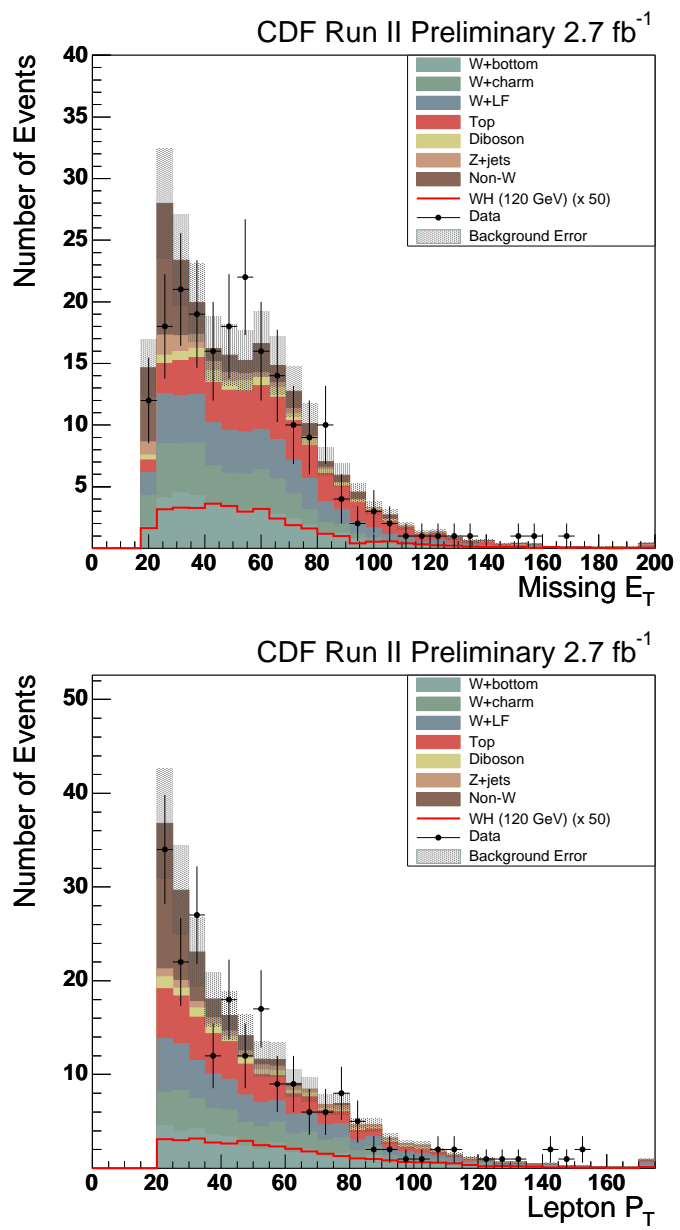

Figure 8.5: Distributions of the $\mathbb{E}_{T}$ and lepton $p_{T}$ for one tag isolated track events. The plots show the $W H$ signal at 50 times it's Standard Model expectation. 


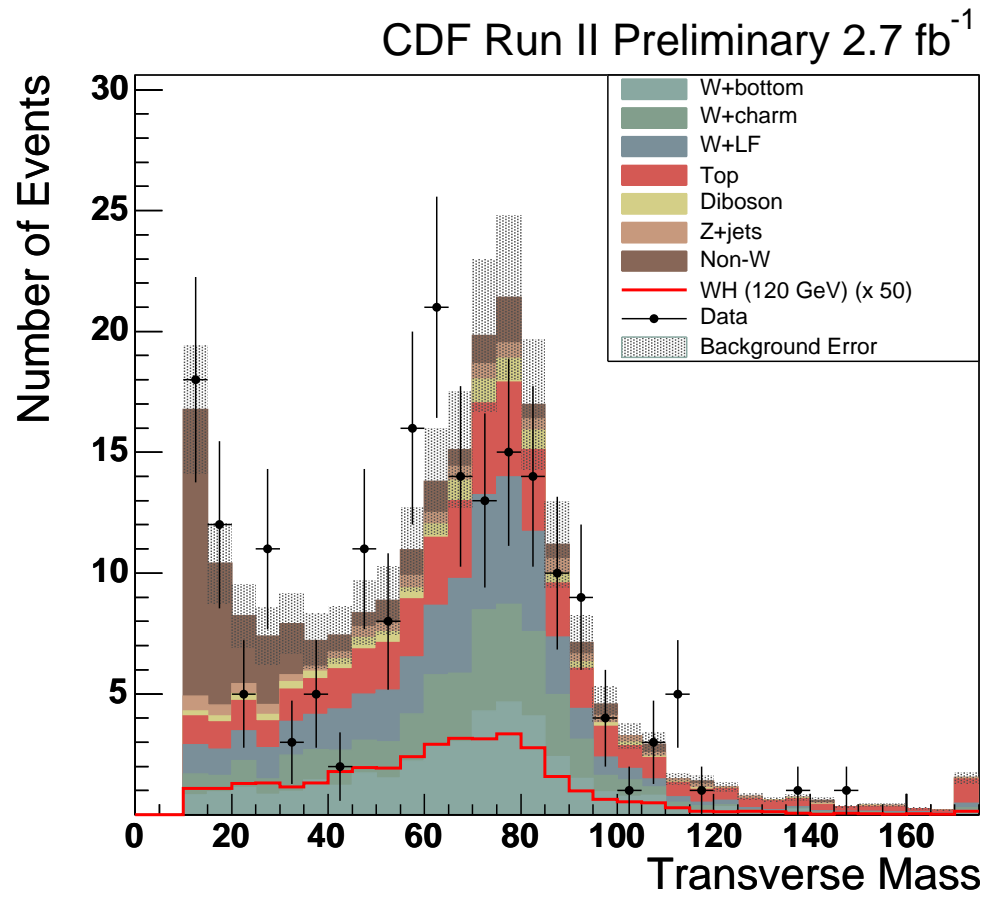

Figure 8.6: Distributions of the $M_{T}$ for one tag isolated track events. The plots show the $W H$ signal at 50 times it's Standard Model expectation. 


\subsection{Two SECVTX Tag Plots}

Statistics in the two SECVTX tag sample prevent a strict evaluation of modeling. Figures 8.7 through 8.9 show the transverse energies and transverse mass for two SECVTX tag events. The plot show no evidence for significant deviations of the observed data from the model. 

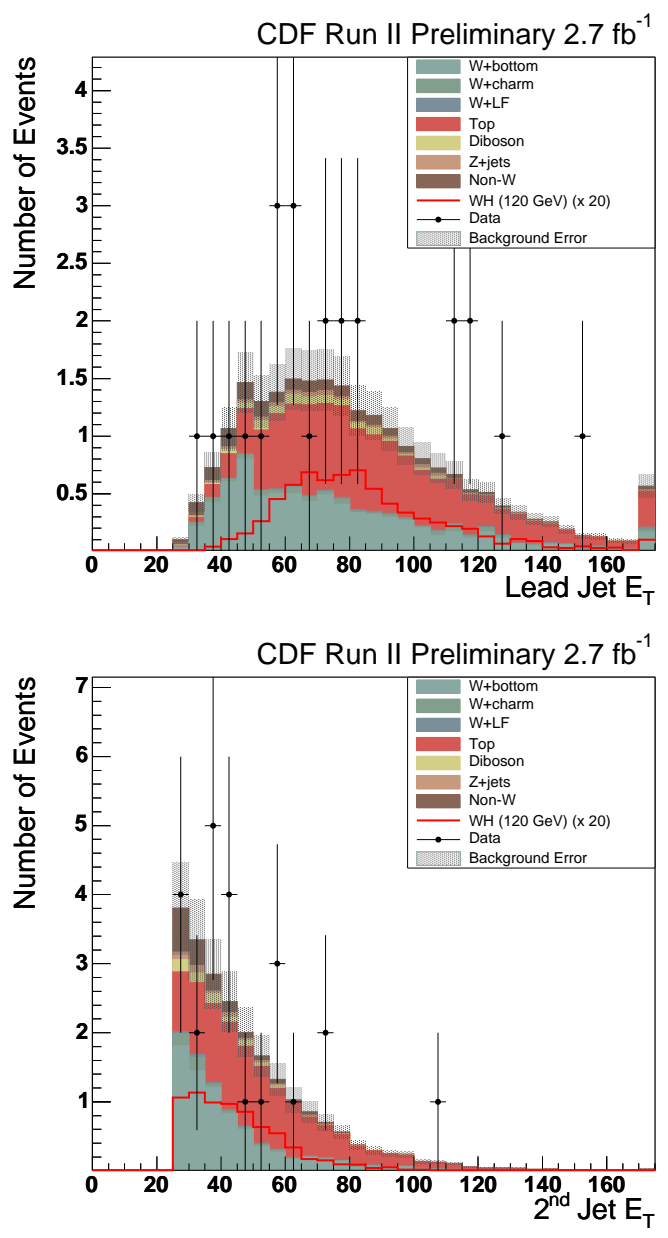

Figure 8.7: Distributions of the lead jet $E_{T}$ and second jet $E_{T}$, for two SECVTX tag isolated track events. The plots show the $W H$ signal at 50 times it's Standard Model expectation. 

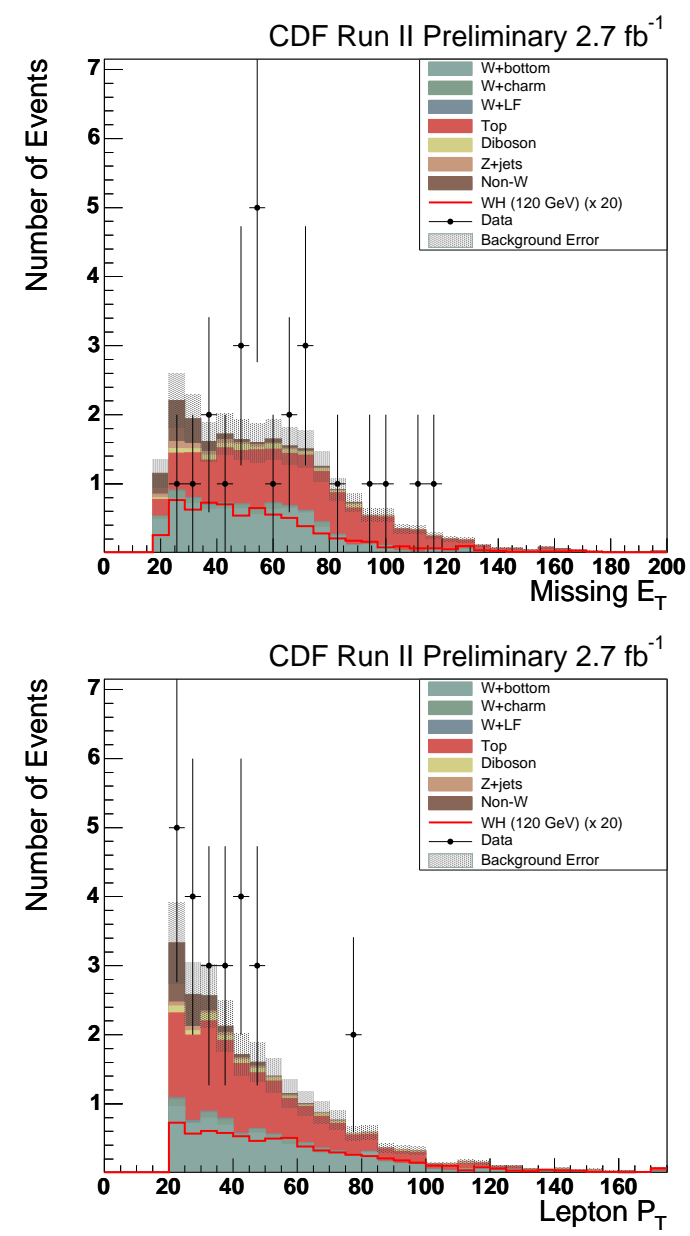

Figure 8.8: Distributions of the $E_{T}$ and lepton $p_{T}$ for two SECVTX tag isolated track events. The plots show the $W H$ signal at 50 times it's Standard Model expectation. 


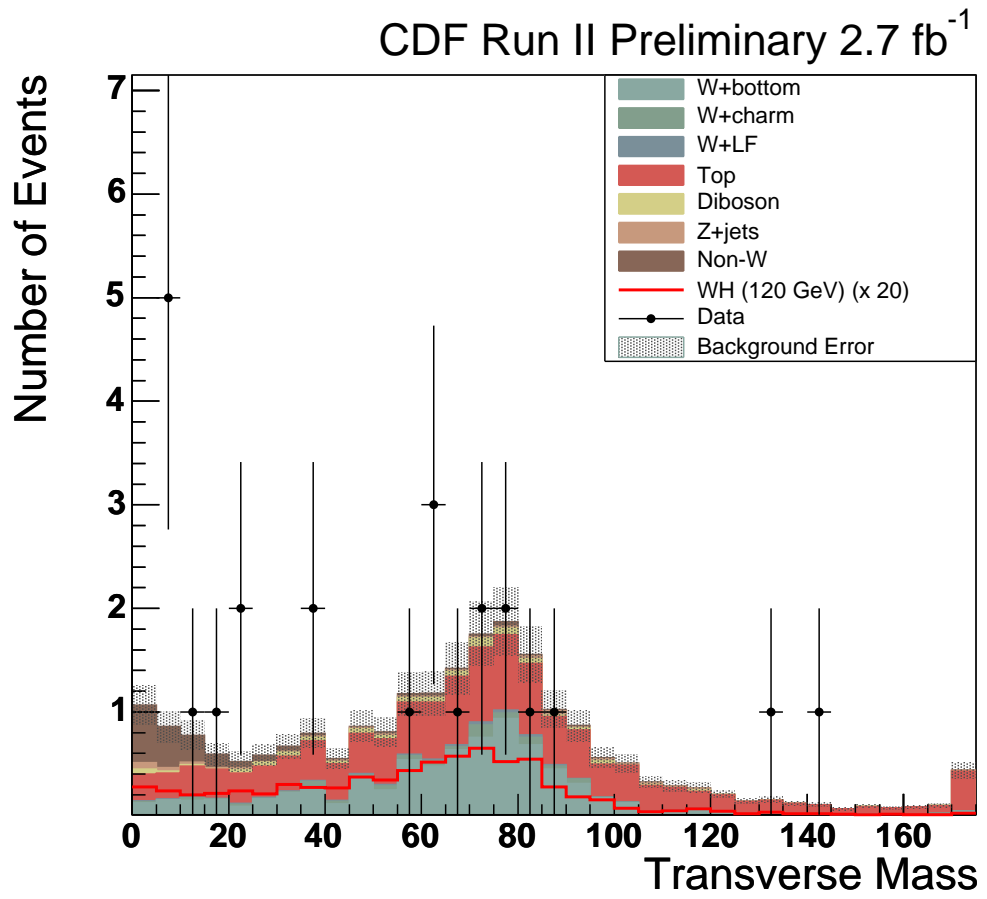

Figure 8.9: Distributions of the $M_{T}$ for two SECVTX tag isolated track events. The plots show the $W H$ signal at 50 times it's Standard Model expectation. 


\section{CHAPTER 9}

\section{NEURAL NETWORK DISCRIMINANT}

We want to exploit our successful modeling of background kinematics and use shape fitting to enhance our sensitivity. The dijet mass shape offers good discrimination between signal and background events, but it only uses some of the event information. The dijet mass neglects the $W$ decay and potential correlations between the $W-H$ system. It is important to use as much information as possible because Higgs events look so similar to their major backgrounds of $W+b \bar{b}$ and $t \bar{t}$. Multivariate techniques can incorporate information from several kinematic variables into a single shape optimized to create a signal enriched region. We use an Artificial Neural Network to develop a multivariate discriminant optimized for $W H$ sensitivity.

\subsection{Artificial Neural Network Overview}

Artificial Neural Networks (ANNs) are multivariate functions developed through an iterative training process. The networks consist of a series of nodes with weighted interconnections. We use a feed-forward network that allows information to flow from the input nodes to the output nodes. The input nodes are a set of kinematic variables that describe each event. The input and output nodes are connected through a layer of hidden nodes. The hidden nodes and output nodes apply a sigmoid function to 
weighted combinations of their inputs. The network output falls between zero and one. We use the JETNET [38] artificial neural network package to implement and train our networks.

Training optimizes the ability of the neural network to classify events as signal or background. We train the neural network by iterating over a set of events associated with target output values called the training sample. The target is set to one for signal events and zero for background events. The network uses the values of the kinematic variables in each event to calculate an output value. After comparing the network output to the target, we use back-propagation to update the network connection weights and move the output closer to the target. Back-propagation updates the weights by an amount proportional to the output distance from the target.

Neural networks have been used as a tool in several high-energy physics analyses. CDF previously used neural networks to measure the $t \bar{t}$ cross section without $b$ tagging [39]. The top cross section measurement demonstrated the applicability of neural networks to $W+$ jets and $t \bar{t}$ events. Our analysis also applies a different neural network to the same processes, but classifies them both as backgrounds.

\subsection{Neural Network Description}

Our Neural Network configuration has 6 input variables, 11 hidden nodes, and 1 output node. We optimized our choice of the neural network input variables by testing numerous network configurations. The optimization procedure began by training approximately 70 separate one-input neural networks that each used a distinct input variable. The procedure ranked each network according to it's total classification error, selected the network with the lowest classification error, and identified the 
network's input as the most sensitive variable. The next stage of the optimization trained separate two-input networks for each pairing of the most sensitive variable with one other. The procedure stopped when adding an additional variable did not reduce the total classification error. The optimal network from this procedure has the following 6 input variables:

$M_{j j_{+}}$: This variable is the invariant mass calculated from the two jets. Furthermore, if there are additional loose jets present $\left(12<E_{T}<20 \mathrm{GeV}\right.$ or $|\eta|<2.4$ and $E_{T}>12$ ), the loose jet that is closest to one of the two jets is included in this invariant mass calculation, if the separation between that loose jet and one of the jets is $\Delta R<0.9$.

$\sum E_{T}$ (Loose Jets): This variable is the scalar sum of the loose jet transverse energy.

$p_{T}$ Imbalance: This variable expresses the difference between the scalar sum of the transverse momenta of all measured objects and the $E_{T}$. Specifically, it is calculated as $P_{T}\left(\right.$ jet $\left._{1}\right)+P_{T}\left(\right.$ jet $\left._{2}\right)+P_{T}(l e p)-\not_{T}$.

$M_{l \nu j}^{\min }$ : This is the invariant mass of the lepton, $\mathbb{E}_{T}$, and one of the two jets, where the jet is chosen to give the minimum invariant mass. For this quantity, the $p_{z}$ component of the neutrino is ignored.

$\Delta R\left(\right.$ lepton- $\left.\nu_{\max }\right):$ This is the $\Delta R$ separation between the lepton and the neutrino, where the $p_{z}$ of the neutrino is taken from by choosing the solutions from the quadratic equations for the $W$ mass $\left(80.42 \mathrm{GeV} / \mathrm{c}^{2}\right)$ constraint with the largest $\left|p_{z}\right|$. 
$P_{T}(W+H)$ : This is the total transverse momentum of the $W$ plus two jets system, $P_{T}\left(l \overrightarrow{e p}+\vec{\nu}+j \overrightarrow{e t}_{1}+j \overrightarrow{e t}_{2}\right)$.

Our training sample has $25 \%$ signal, $75 \%$ background events. The background sample was split evenly between $W+\mathrm{bb}, t \bar{t}$, and single top $t$-channel. We varied the sample composition to try different mixes of signal and background but we found no significant changes in sensitivity.

We use the same topology and input variables to train separate neural networks for all Higgs mass choices. The Higgs Monte Carlo samples range from $M(H)=100$ to $150 \mathrm{GeV} / \mathrm{c}^{2}$ in $5 \mathrm{GeV} / \mathrm{c}^{2}$ increments. At each Higgs mass, we use the same neural network for tight lepton and isolated track events.

Figures 9.1 through 9.3 show the 6 neural network input variables in the for isolated track events in the pretag control region. Figures 9.4 and 9.5 show the same distributions in the one tag sample. The distributions show that the neural network inputs are well modeled.

\subsection{Neural Network Output}

Figures 9.7 through 9.9 show the neural network output distributions for isolated track events (left in each figure) and tight lepton events (right in each figure). The analysis combines searches in both the tight lepton channels and isolated track channels, and the figures show the NN output distributions for both channels. Backgroundlike events have a neural network output near zero. Signal-like events have a neural network output near one. We produce separate distributions for each mass-specific neural network. The 

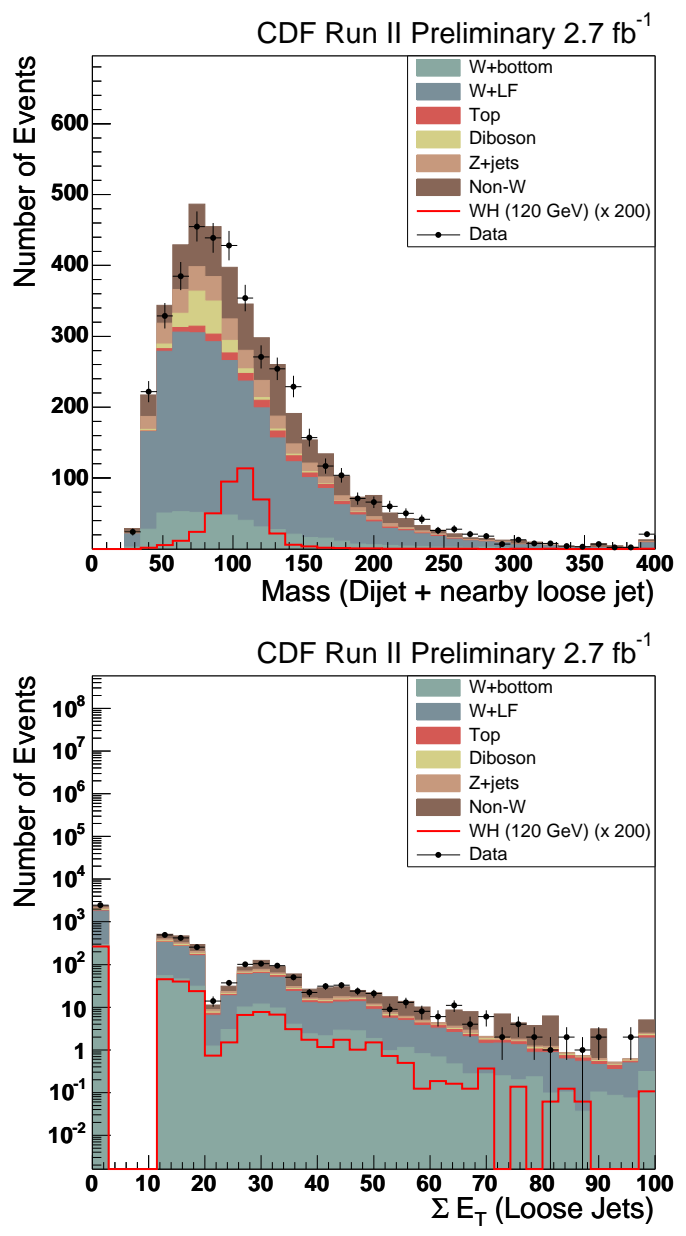

Figure 9.1: Neural Network input distributions for isolated track $W+2$ jets events in the pretag control region. The distributions shown are $M_{j j+}$ (left), $\sum E_{T}$ (Loose Jets) (left). 

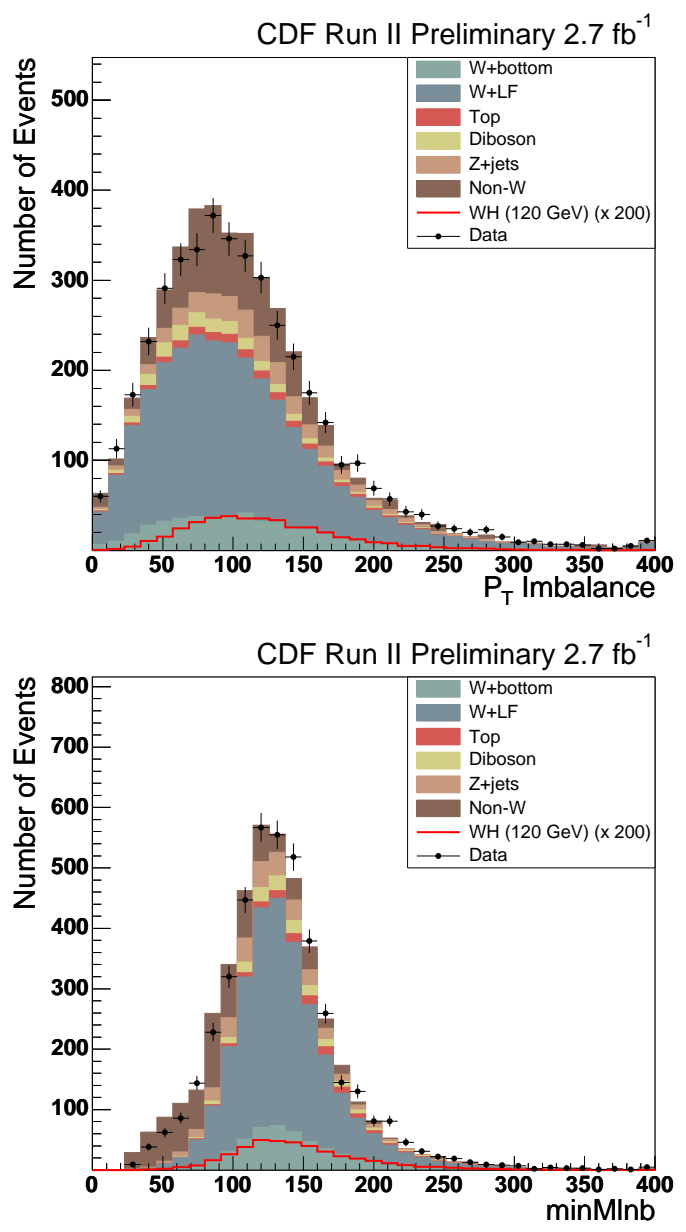

Figure 9.2: Neural Network input distributions in isolated track pretag $W+2$ jet events. The distributions shown are $p_{T}$ Imbalance (left) and $M_{l \nu j}^{\min }$ (right). 

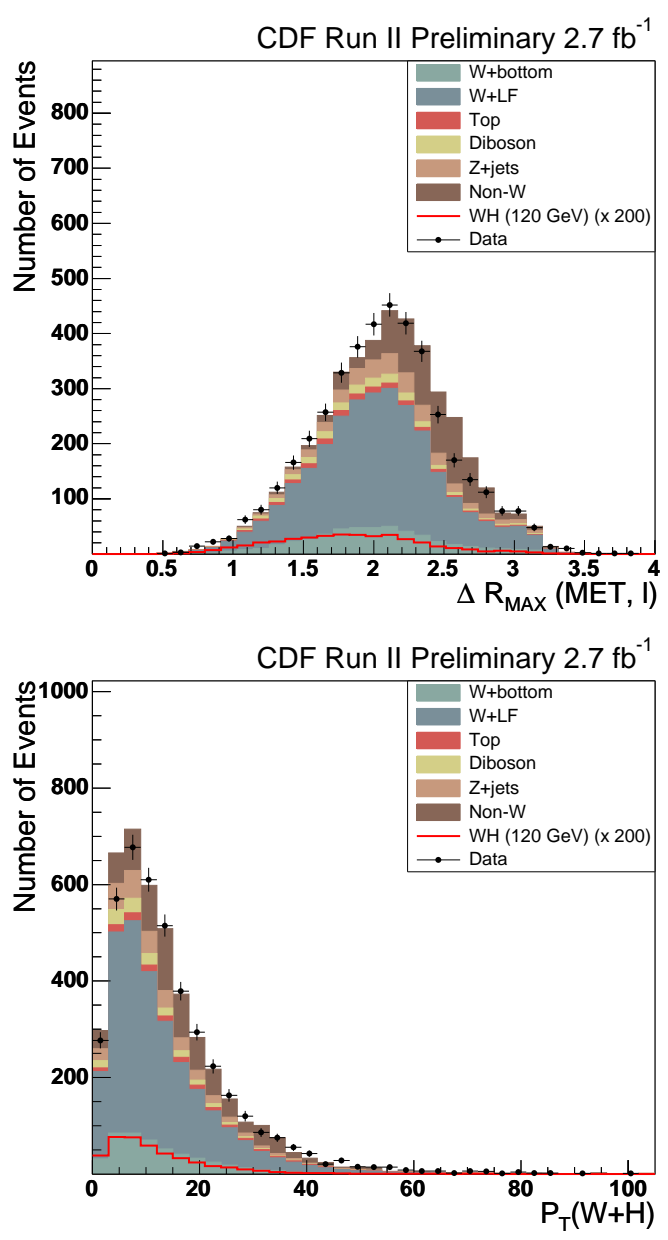

Figure 9.3: Neural Network input distributions in isolated track pretag $W+2$ jet events. The distributions shown are $\Delta R$ (lepton- $\left.\nu_{\max }\right)$ (left) and $P_{T}(W+H)$ (right). 

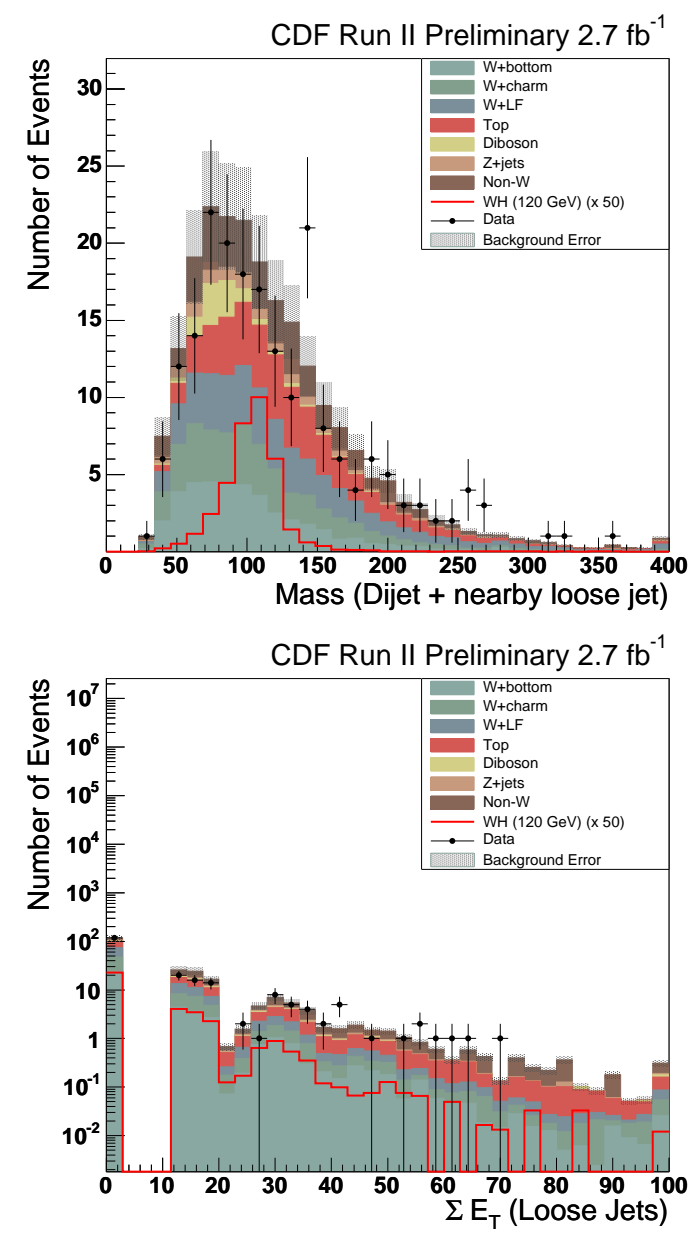

Figure 9.4: Neural Network input distributions for isolated track one tag $W+2$ jets events. The distributions shown are $M_{j j+}$ (left), $\sum E_{T}$ (Loose Jets) (left). 

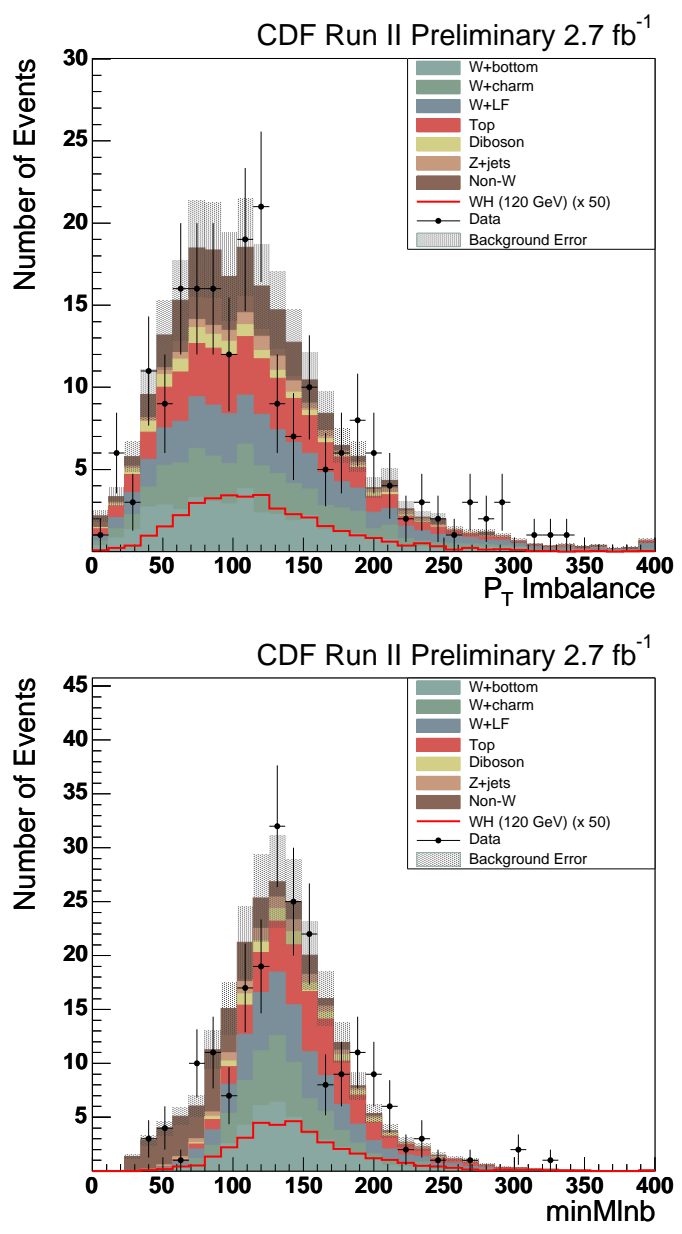

Figure 9.5: Neural Network input distributions in isolated track one tag $W+2$ jet events. The distributions shown are $p_{T}$ Imbalance (left) and $M_{l \nu j}^{\min }$ (right). 

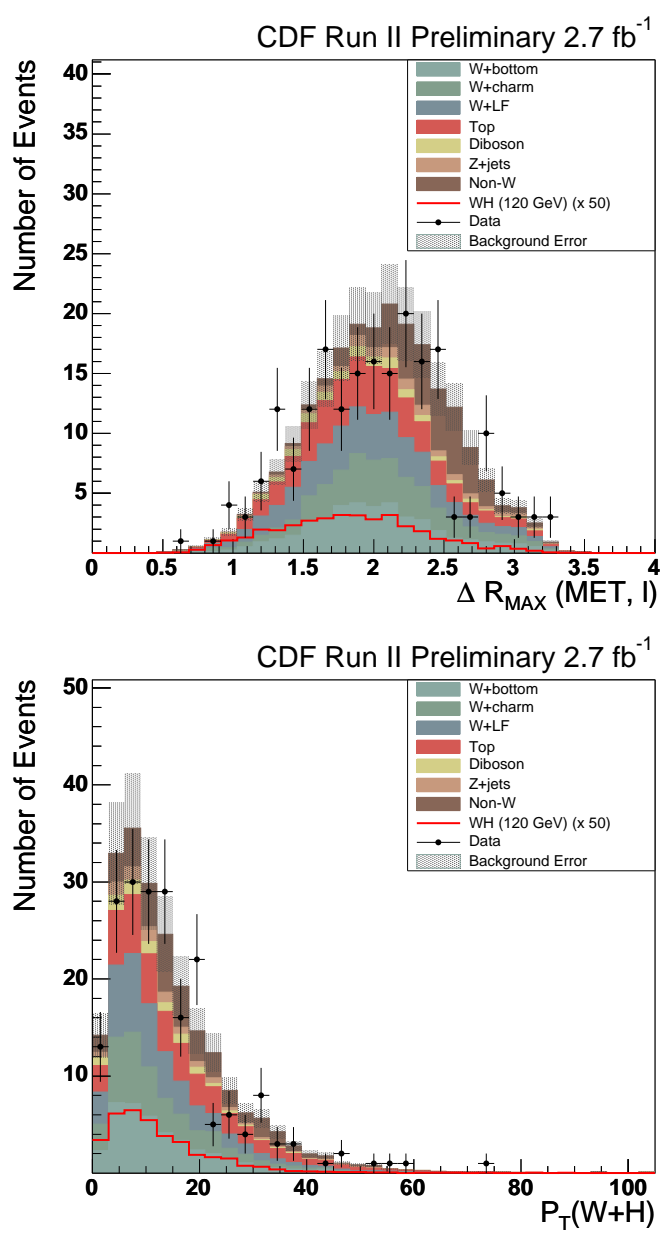

Figure 9.6: Neural Network input distributions in isolated track one tag $W+2$ jet events. The distributions shown are $\Delta R$ (lepton- $\left.\nu_{\max }\right)$ (left) and $P_{T}(W+H)$ (right). 

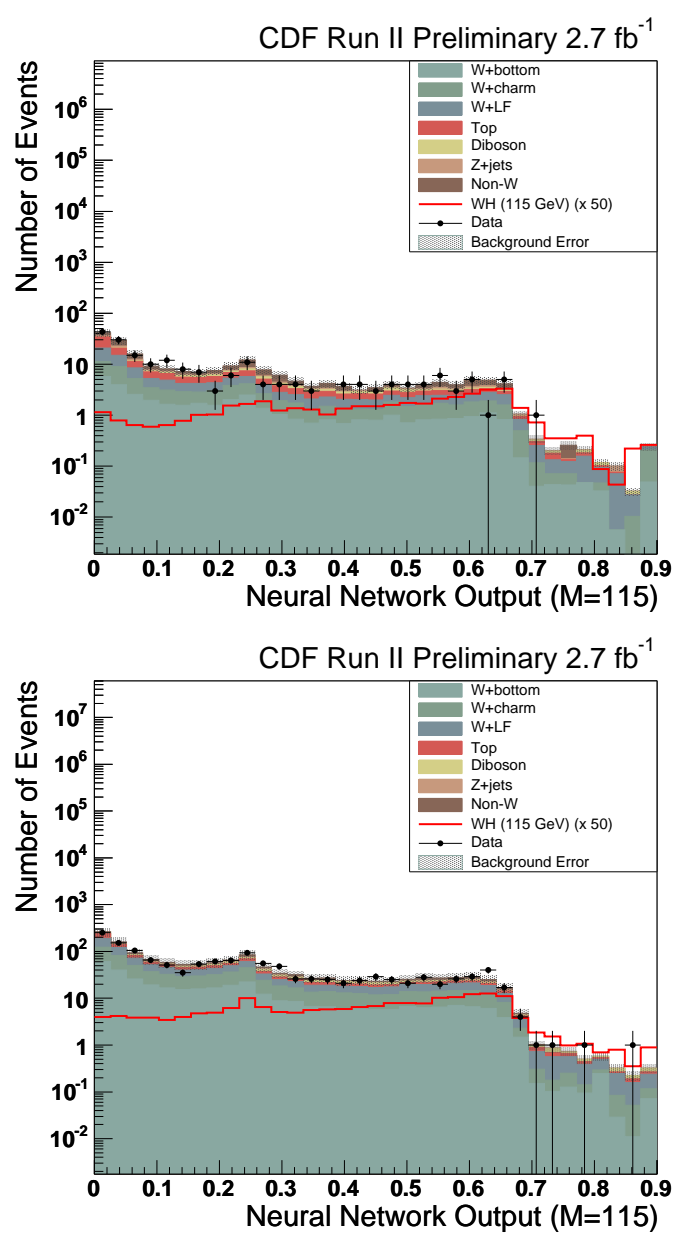

Figure 9.7: Neural Network output distributions for events with one secvtx tag. The plots show isolated track events (left) and lepton triggered events (right). 

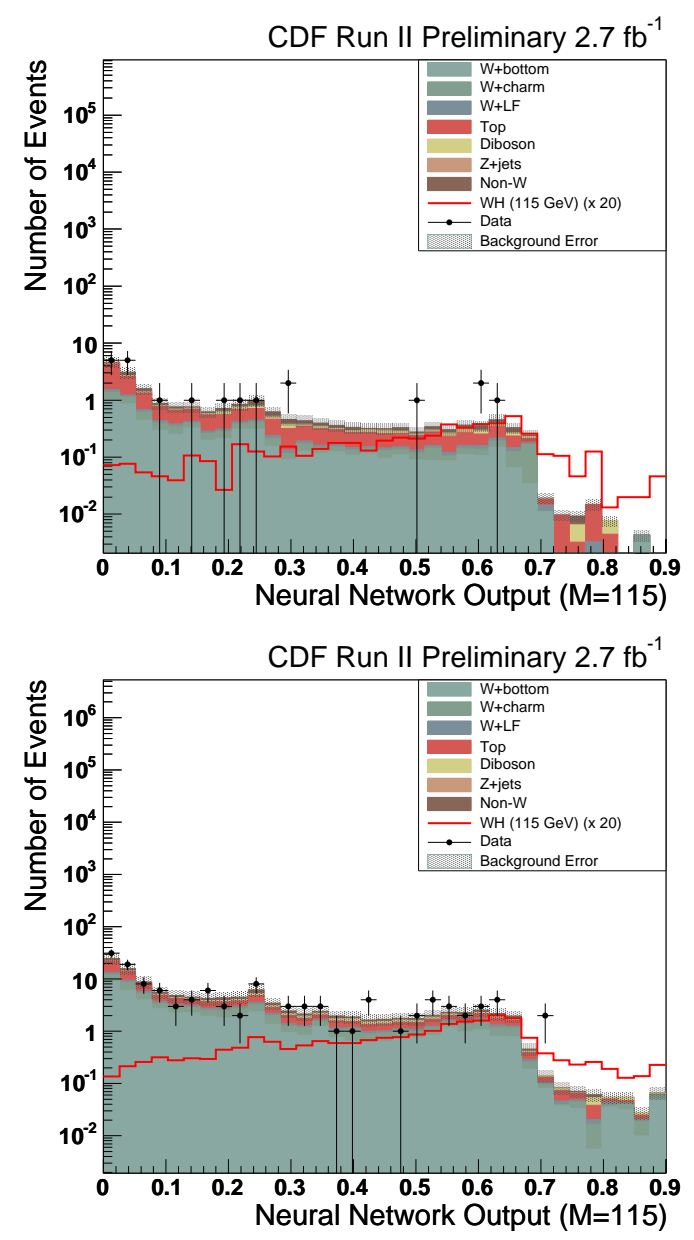

Figure 9.8: Neural Network output distributions for events with one secvtx tag. The plots show isolated track events (left) and lepton triggered events (right). 

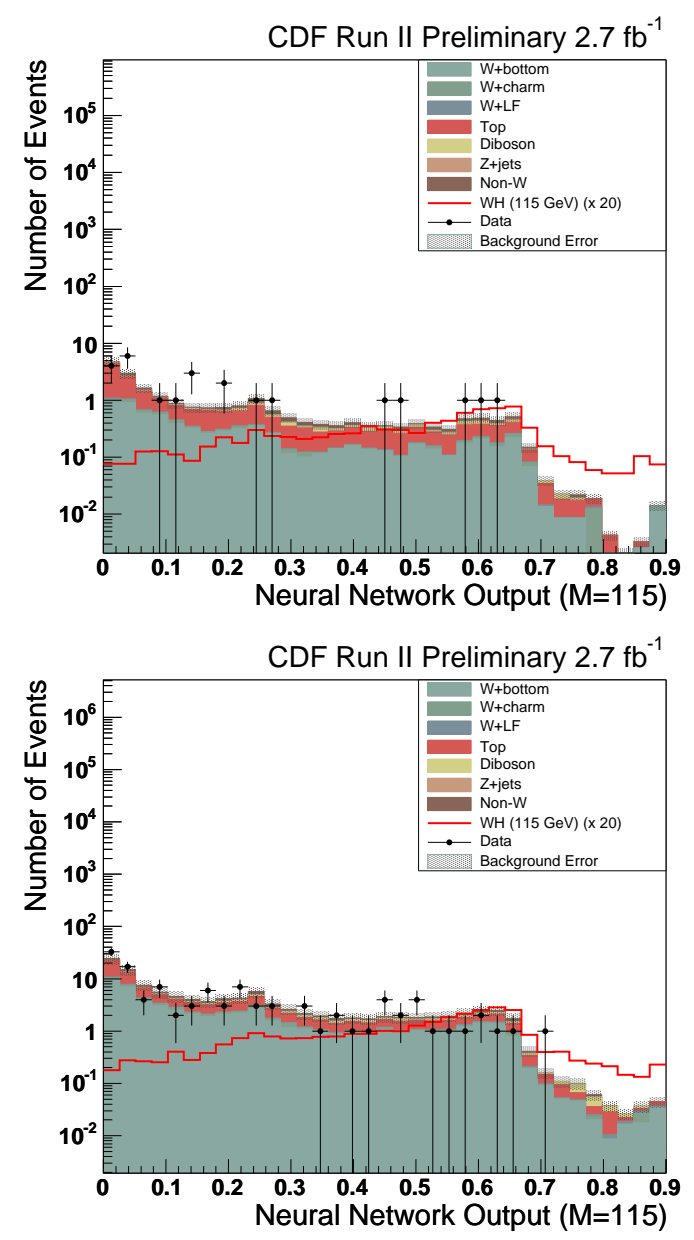

Figure 9.9: Neural Network output distributions for events with one secvtx tag. The plots show isolated track events (left) and lepton triggered events (right). 


\section{CHAPTER 10}

\section{RESULTS}

\subsection{Binned Likelihood Technique}

We search for Higgs bosons by comparing our observed distribution of events to a background-only hypothesis (null hypothesis) and to a hypothesis with background and signal. The procedure is similar to the one described in "Confidence Level Computation for Combining Searches with Small Statistics" [40], and used in CDF's search for single top production [24]. In the null hypothesis, the number of events in each bin of neural network output is the expected number of background events. In the signal hypothesis, the number of expected events is the total number of signal plus background events. The number of estimated events in each bin $i$ follows Poisson statistics. The mean of the Poisson, $\mu_{i}$ is the estimated number of events. The probability to observe a number of events $n_{i}$ in a given bin is:

$$
P_{i}\left(n_{i}, \mu_{i}\right)=\frac{\mu_{i}^{n_{i}} e^{-\mu_{i}}}{n_{i} !} \quad\left(i=1,2, \cdots, N_{\mathrm{bin}}\right)
$$

where

$$
\begin{array}{r}
\mu_{i}=n_{\text {Background }} \quad \text { (null hypothesis) } \\
\mu_{i}=n_{\text {Signal }}+n_{\text {Background }} \quad \text { (test hypothesis) }
\end{array}
$$


The overall probability that a given observed distribution is consistent with either hypothesis is given by:

$$
L=\prod_{i=1}^{N_{\text {bin }}} P_{i}\left(n_{i}, \mu_{i}\right)=\prod_{i=1}^{N_{\text {bin }}} \frac{\mu_{i}^{n_{i}} e^{-\mu_{i}}}{n_{i} !} .
$$

Equation 10.4 gives the probability for an observed distribution to agree with exact background prediction. We accommodate the uncertainties in our background estimate using truncated Gaussian constraints. Truncated Guassians extend only from $-1 \sigma$ to $+1 \sigma$. The allow the systematics to fluctuate smoothly between $\pm 1 \sigma$, but not beyond. The likelihood with constrained uncertainties is,

$$
\begin{aligned}
L & =\int_{N^{Q C D}} \int_{N^{T O P}} \int_{N^{W H}} \prod_{i=1}^{N_{\mathrm{bin}}} \frac{\mu_{i}^{n_{i}} e^{-\mu_{i}}}{n_{i} !} \\
& \times G\left(n^{W+J e t s}, \sigma^{n+J e t s}\right) G\left(n^{Q C D}, \sigma^{Q C D}\right) \\
& \times G\left(n^{T O P}, \sigma^{T O P}\right) G\left(n^{W H}, \sigma^{W H}\right) \\
& \times d N^{Q C D} d N^{T O P} d N^{W H} d N^{W+J e t s}
\end{aligned}
$$

where $G\left(N^{b k g}, \sigma^{b k g}\right)$ are the truncated Gaussian representing the uncertainty on each of the backgrounds and the signal. We assume a flat prior when calculating the likelihood, and integrate over all systematic parameters. We use log-likelihoods

$$
\begin{aligned}
P\left(\text { data } \mid H_{s+b}\right) & =-\ln [L(s+b)] \\
P\left(\text { data } \mid H_{b}\right) & =-\ln [L(b)]
\end{aligned}
$$

to construct a likelihood ratio,

$$
Q=\frac{P\left(\operatorname{data} \mid H_{s+b}\right)}{P\left(\operatorname{data} \mid H_{b}\right)}
$$


$Q$ is the test statistic that we use to compare difference pseudo experiments. The confidence level to exclude signal is,

$$
C L=P\left(Q \leq Q_{o b s}\right)
$$

In this formulation, $\mathrm{CL}=0.05$ corresponds to $95 \%$ confidence that a signal is not present in the data at the assumed rate. We use this confidence level to set limits on the $W H$ production cross section times branching ratio.

We measure our expected sensitivity using pseudo-experiments with pseudo data constructed from a sum of background templates. The amount of each background in a particular pseudo-experiment is allowed to fluctuate within the uncertainty on the background estimate.

Each combination of lepton and tag category is treated as a separate channel in the likelihood calculation. We correlate sources of systematic uncertainty that impact many channels, such as b-tag scale factor uncertainty and heavy flavor fraction uncertainty.

\subsection{Limit on Higgs Boson Production Rate}

We search for an excess of Higgs signal events in our neural network output distributions using our binned likelihood technique. We find no evidence of a signal excess and we set upper limits on the $W H$ production cross section times $H \rightarrow b \bar{b}$ branching ratio. Figure 10.1 and Table 10.1 show the expected and observed limits

on $\sigma(p \bar{p} \rightarrow W H) \cdot \mathcal{B}(H \rightarrow b \bar{b})$. The limits are expressed a ratio to the Standard Model cross section times branching ratio (x SM). The observed limit at a mass of 


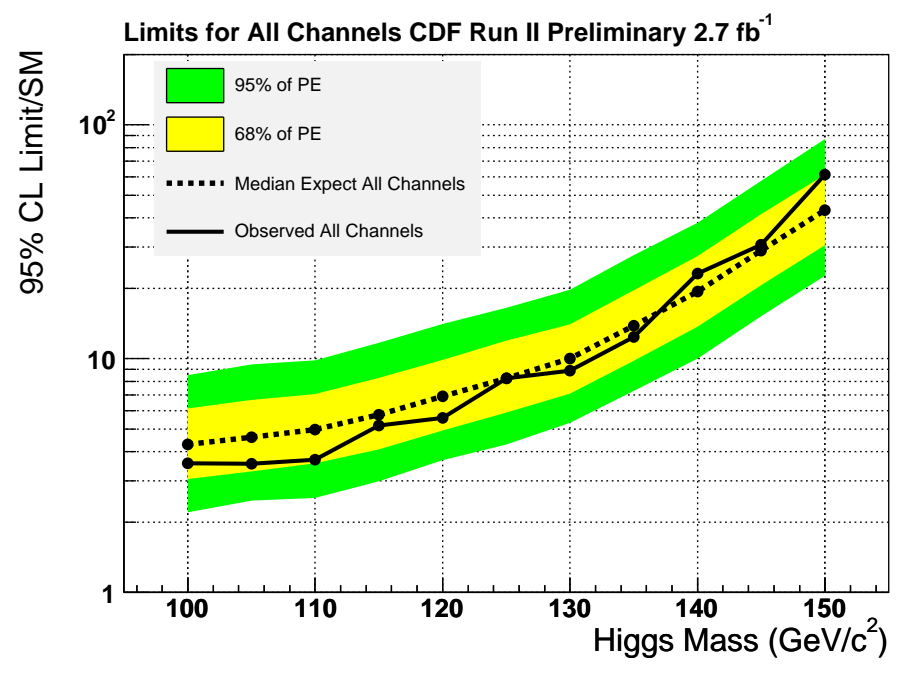

Figure 10.1: 95\% confidence level upper limit on $\sigma(p \bar{p} \rightarrow W H) \cdot \mathcal{B}(H \rightarrow b \bar{b})$, expressed as a ratio to the Standard Model expectation. The limits were obtained using an integrated luminosity of $2.7 \mathrm{fb}^{-1}$ and analyzing both lepton triggered and $\not_{T}+2$ jet triggered events. The dashed line indicates the median expected limit. The yellow and green bands indicate the regions encompassing the limits in $68 \%$ and $95 \%$ of pseudo experiments. The solid line shows the observed limts.

$115 \mathrm{GeV} / \mathrm{c}^{2}$ is 5.2 (5.8 expected) x SM. The observed limits agree well with the expected limits. 


\begin{tabular}{|c|c|c|}
\hline \multicolumn{3}{|c|}{ CDF Run II Preliminary $2.7 \mathrm{fb}^{-1}$} \\
Limits for Combined Lepton and Tag Categories \\
\hline $\mathrm{M}(\mathrm{H})\left(\mathrm{GeV} / \mathrm{c}^{2}\right)$ & Observed Limit & Expected Limit \\
\hline 100 & 3.6 & 4.3 \\
105 & 3.6 & 4.6 \\
110 & 3.7 & 5.0 \\
115 & 5.2 & 5.8 \\
120 & 5.6 & 6.9 \\
125 & 8.2 & 8.2 \\
130 & 8.9 & 10.0 \\
135 & 12.4 & 13.8 \\
140 & 23.1 & 19.4 \\
145 & 30.6 & 28.9 \\
150 & 61.1 & 43.2 \\
\hline
\end{tabular}

Table 10.1: Expected and observed limits as a function of Higgs mass for the combined search of Tight Lepton and Isotrk events, including all tag categories. The Higgs masses are in units of $\mathrm{GeV} / \mathrm{c}^{2}$, and the limits are expressed as ratio of experimental limit to the theoretical production rate. 


\section{CHAPTER 11}

\section{DISCUSSION AND CONCLUSION}

Our $W H$ search represents a substantial improvement in sensitivity over prior searches. The increase in sensitivity comes not only from an increase in integrated luminosity, but also from improvements in analysis techniques. The $1 \mathrm{fb}^{-1} \mathrm{WH}$ result did not use a multivariate technique, and did not analyze events recorded on $E_{T}+2$ Jets trigger [26]. The neural network improves the analysis sensitivity by $10 \%$ compared to fitting the dijet mass alone. Including isolated track events increases the acceptance by $25 \%$ and yields a $15 \%$ improvement in sensitivity. Both improvements combine for an overall $26 \%$ increase in sensitivity. The $1 \mathrm{fb}^{-1}$ techniques would need a dataset of $4.28 \mathrm{fb}^{-1}\left(1.58 \mathrm{fb}^{-1}\right.$ more $)$ to achieve comparable sensitivity. Increasing our dataset by $1.58 \mathrm{fb}^{-1}$ would require approximately 1 year of additional Tevatron collisions at current luminosity.

Our $W H$ search is one of the most sensitive low-mass Higgs searches at the Tevatron. Figure 11.1 shows that it is more sensitive than CDF's other low-mass search channels, such as $V H \rightarrow \mathbb{E}_{T}+b b$ and $Z H \rightarrow l \nu b b$ [41]. It has comparable or better sensitivity to a $W H$ search using identical luminosity and event selection but different multivariate technique [42]. The different multivariate techniques achieved comparable sensitivity by looking at different event features. We combined our neural network 


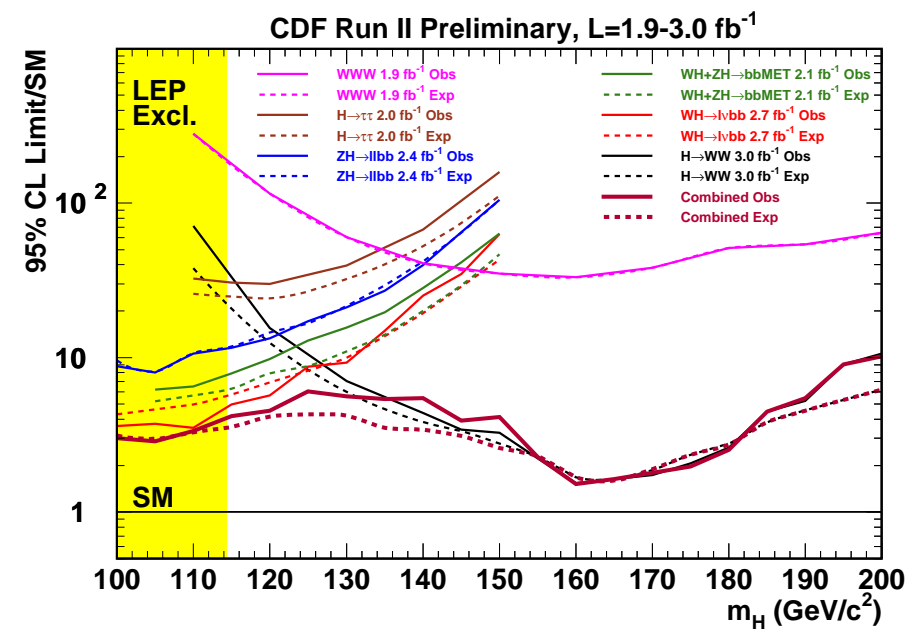

Figure 11.1: 95\% confidence level upper limit on various channels of Higgs production, expressed as a ratio to the Standard Model expectation. The limits were obtained using an luminosities from of $1.0-3.0 \mathrm{fb}^{-1}$ The dashed line indicates the median expected limit. The figures shows limits for separate search channels, and the CDF combination of the separate searches. 
discriminant with the other $W H$ search in a single "super-discriminant". The super discriminant incorporated the strengths of each analysis and lead to an improvement of $10 \%$ in sensitivity [43]. The combined $W H$ search was the most sensitive low-mass Higgs search at the Tevatron.

Despite these improvements, the $W H$ channel alone will not be sensitive enough to discover the Higgs with the full anticipated Tevatron dataset of approximately 6 $\mathrm{fb}^{-1}$ (double the current dataset). The full dataset will improve the sensitivity by $\sqrt{L_{\text {new }} / L_{\text {old }}}=1.4$. Even with potential improvements, it will be highly unlikely to achieve Standard Model sensitivity for low-mass Higgs with only the $W H$ channel. The Tevatron can improve sensitivity by combining analysis channels and combining datasets from the two experiments. Such combinations have already been done, and offer improvements of roughly $60 \%$ [41]. These combinations can bring the Tevatron to Standard Model sensitivity only if the individual analyses incorporate further improvements.

The Tevatron excluded Higgs masses near $170 \mathrm{GeV} / \mathrm{c}^{2}$ in the summer of 2008 in a search for $H \rightarrow W^{+} W^{-}[41]$. The exclusion was the first direct limit on Higgs production since the LEP experiments. This result provides an model of success for low mass Higgs searches. The $H \rightarrow W^{+} W^{-}$analysts achieved their sensitivity by expanding lepton identification, analyzing samples with weaker signal-to-background ratios, incorporating several multivariate techniques into a single analysis, and combining CDF and DØ datasets. Their success makes the low-mass searches optimistic about the prospect of Higgs sensitivity. 


\section{BIBLIOGRAPHY}

[1] ALEPH, CDF, D0, DELPHI, L3, OPAL, SLD Collaboration, Precision electroweak measurements and constraints on the standard model, arXiv:0811.4682v1.

[2] Particle Data Group Collaboration, C. A. et. al., Review of particle physics, Phys. Lett. B 667 (2008).

[3] F. Halzen and A. D. Martin, Quarks and Leptons: an Introductory Course in Modern Particle Physics, . New York, Usa: Wiley (1984) 396p.

[4] LEP Working Group for Higgs boson searches Collaboration, R. Barate et. al., Search for the standard model Higgs boson at LEP, Phys. Lett. B565 (2003) 61-75 [hep-ex/0306033].

[5] Fermilab Beams Division Collaboration, Tevatron Run 2 handbook, 1998.

[6] CDF Collaboration, F. Abe et. al., The CDF detector: an overview, Nucl. Instr. Meth. A271 (1988) 387-403.

[7] D. Acosta et. al., The performance of the CDF luminosity monitor, Nucl. Instrum. Meth. A494 (2002) 57-62.

[8] CDF Collaboration, A. Sill, CDF run II silicon tracking projects, Nucl. Instrum. Meth. A447 (2000) 1-8.

[9] CDF Collaboration, K. T. Pitts, The CDF central outer tracker, Nucl. Phys. Proc. Suppl. 61B (1998) 230-235.

[10] CDF Collaboration, A. A. Affolder et. al., CDF central outer tracker, Nucl. Instrum. Meth. A526 (2004) 249-299.

[11] CDF Collaboration, L. Balka et. al., The CDF central electromagnetic calorimeter, Nucl. Instrum. Meth. A267 (1988) 272.

[12] CDF Collaboration, S. Bertolucci et. al., The CDF central and endwall hadron calorimeter, Nucl. Instrum. Meth. A267 (1988) 301. 
[13] CDF Collaboration, M. G. Albrow et. al., The CDF plug upgrade electromagnetic calorimeter: Test beam results, Nucl. Instrum. Meth. A480 (2002) 524-546.

[14] G. Ascoli et. al., CDF central muon detector, Nucl. Instrum. Meth. A268 (1988) 33.

[15] L. Balka et. al., The cdf central electromagnetic calorimeter, Nucl. Instrum. Methods A267 (1988) 272.

[16] CDF Collaboration, T. Aaltonen et. al., Search for Standard Model Higgs Boson Production in Association with a W Boson at CDF, Phys. Rev. D 78 (2008) 032008 [0803.3493v1].

[17] CDF collaboration Collaboration, D. Acosta et. al., First measurements of inclusive $w$ and $z$ cross sections from run ii of the tevatron collider, Phys. Rev. Lett. 94 (2005) 091803 [hep-ex/0406078].

[18] C. E. Mills, A measurement of the top pair production cross-section in the dilepton channel using lepton plus track selection, .

FERMILAB-THESIS-2007-49.

[19] CDF collaboration Collaboration, D. Acosta et. al., First measurements of inclusive $w$ and $z$ cross sections from run ii of the tevatron collider, 2005.

[20] CDF collaboration Collaboration, F. Abe et. al., The topology of three jet events in $\bar{p} p$ collisions at $\sqrt{s}=1.8$ tev, Phys. Rev. D45 (1992) 1448-1458.

[21] A. Bhatti et. al., Determination of the jet energy scale at the collider detector at fermilab, Nucl. Instrum. Methods A566 (2006) 375-412 [hep-ex/0510047].

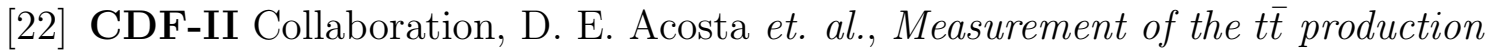
cross section in $p \bar{p}$ collisions at $\sqrt{s}=1.96$ TeV using kinematic fitting of b-tagged lepton + jet events, Phys. Rev. D71 (2005) 072005 [hep-ex/0409029].

[23] CDF collaboration Collaboration, A. Abulencia et. al., Measurement of the $t \bar{t}$ production cross section in $p \bar{p}$ collisions at $\sqrt{s}=1.96$-tev using lepton + jets events with jet probability b- tagging, Phys. Rev. D74 (2006) 072006 [hep-ex/0607035].

[24] CDF Collaboration Collaboration, T. Aaltonen et. al., Evidence for single top produciton at cdf, Phys. Rev. Lett. XXX (2008) XXX.

[25] CDF Collaboration Collaboration, T. Aaltonen et. al., Search for the higgs boson in events with missing transverse energy and $b$ quark jets produced in $p$ anti-p collisions at $s^{* *}(1 / 2)=1.96$ tev, Phys. Rev. Lett. 100 (2008) 211801. 
[26] CDF collaboration Collaboration, T. Aaltonen et. al., Search for Standard Model Higgs Bosons Produced in Association with $W$ Bosons, Phys. Rev. Lett. 100 (2008) 041801 [arXiv:0710.4363 [hep-ex]].

[27] CDF collaboration Collaboration, A. Abulencia et. al., Measurement of the tanti-t production cross section in $p$ anti-p collisions at $\sqrt{s}=1.96$-tev, Phys. Rev. Lett. 97 (2006) 082004 [hep-ex/0606017].

[28] M. L. Mangano, M. Moretti, F. Piccinini, R. Pittau and A. D. Polosa, ALPGEN, a generator for hard multiparton processes in hadronic collisions, JHEP 07 (2003) 001 [hep-ph/0206293].

[29] M. L. Mangano, M. Moretti, F. Piccinini, R. Pittau and A. D. Polosa, Alpgen, a generator for hard multiparton processes in hadronic collisions, J. High Energy Phys. 07 (2003) 001 [hep-ph/0206293].

[30] G. Corcella et. al., Herwig 6.4 release note, hep-ph/0201201.

[31] J. Campbell and R. K. Ellis, Next-to-leading order corrections to $w+$ 2jet and $z+$ 2jet production at hadron colliders, Phys. Rev. D65 (2002) 113007 [hep-ph/0202176].

[32] M. Cacciari, S. Frixione, M. L. Mangano, P. Nason and G. Ridolfi, The t anti-t cross-section at 1.8-tev and 1.96-tev: A study of the systematics due to parton densities and scale dependence, J. High Energy Phys. 04 (2004) 068 [hep-ph/0303085].

[33] B. W. Harris, E. Laenen, L. Phaf, Z. Sullivan and S. Weinzierl, The fully differential single top quark cross section in next-to-leading order qcd, Phys. Rev. D66 (2002) 054024 [hep-ph/0207055].

[34] T. Sjostrand et. al., High-energy-physics event generation with PYTHIA 6.1, Comput. Phys. Commun. 135 (2001) 238-259 [hep-ph/0010017].

[35] CDF collaboration Collaboration, A. Abulencia et. al., Top quark mass measurement using the template method in the lepton + jets channel at cdf ii, Phys. Rev. D73 (2006) 032003 [hep-ex/0510048].

[36] J. Pumplin et. al., New generation of parton distributions with uncertainties from global qcd analysis, J. High Energy Phys. 07 (2002) 012 [hep-ph/0201195].

[37] A. Bhatti et. al., Determination of the jet energy scale at the Collider Detector at Fermilab, Nucl. Instrum. Meth. A566 (2006) 375-412 [hep-ex/0510047]. 
[38] C. Peterson, T. Rognvaldsson and L. Lonnblad, JETNET 3.0: A versatile artificial neural network package, Comput. Phys. Commun. 81 (1994) 185-220. We use a ROOT-based version of JETNET called Root_Jetnet which can be found online.

[39] CDF Collaboration, D. Acosta et. al., Measurement of the cross section for $t \bar{t}$

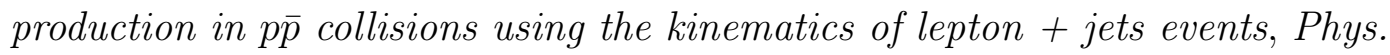
Rev. D72 (2005) 052003 [hep-ex/0504053].

[40] T. Junk, Confidence level computation for combining searches with small statistics, Nucl. Instrum. Meth. A434 (1999) 435-443 [hep-ex/9902006].

[41] CDF collaboration Collaboration, T. A. et al, Combined upper limit on standard model higgs boson productionfor summer 2008, CDF Note $\mathbf{9 5 0 2}$ (2008).

[42] CDF collaboration Collaboration, T. A. et al, Search for standard model higgs boson production in association with a $w$ boson using matrix element and boosted decision tree techniques with $2.7 \mathrm{fb}^{-1}$ of data., CDF Note $\mathbf{9 4 6 3 .}$

[43] CDF collaboration Collaboration, T. A. et al, Combined $w h \rightarrow l \nu b \bar{b}$ search with $2.7 \mathrm{fb}^{-1}$ of data, CDF Note $\mathbf{9 5 9 6 .}$ 